\title{
23. \\ Recherches sur les formes quadratiques à coëfficients et à indéterminées complexes.
}

(Par Mr. G. Lejeune Dirichlet à Berlin.)

\section{Première partie.}

Comme les recherches que nous aurons à exposer dans ce Mémoire, présentent par leur objet et par les résultats auxquels elles conduisent, beaucoup d'analogie avec d'autres recherches déjà publiées *), il convient, avant d'en donner un idée générale, de rappeler en peu de mots la question qui a été traitée dans le Mémoire que nous venons de citer. Le Mémoire dont il s'agit, se rapporte à la théorie des formes quadratiques, théorie qui, préparée par quelques énoncés de Fermat et par les ingénieuses recherches d'Euler et définitivement fondée par Lagrange, a reçu plus tard de notables accroissements par les travaux de Legendre et surtout par ceux de Mr. Gaufs, qui y a consacré la plus grande partie de ses „Disquisitiones arithmeticae," en sorte qu'elle constitue aujourd'hui l'une des branches principales de la science des nombres. On sait que les propriétés d'une telle expression dépendent surtout d'un entier qui est une fonction très-simple de ses coëfficients et que par cette raison on nomme le déterminant de la forme quadratique. Quoique le nombre des formes qui ont un même déterminant donné quelconque, positif ou négatif, soit infini, ces formes se réduisent toujours à un nombre limité d'expressions distinctes, c'est-à-dire, non-transformables les unes dans les autres. Cette propriété, capitale dans la matière, a été établie par Lagrange qui a aussi fait connaître les opérations arithmétiques, au moyen desquelles ces formes non-ẻquivalentes peuvent être assignées, lorsque le déterminant est numériquement donné. Mais si ce procédé suffisait pour l'objet auquel son illustre auteur l'avait déstiné, il ne donnait aucune lumière sur la liaison générale qui doit exister entre le déterminant et le nombre des formes distinctes qui

*) Recherches sur diverses applications de l'Analyse infinitésimale à la Théorie des Nombres. Tome XIX et XXI de ce Journal.

Crelle's Journal f. d. M. Bd. XXIV. Heft 4. 
y répondent, et la loi qui exprime cette dépendance et dont la connaissance, outre qu'elle devait présenter beaucoup d'intérêt par elle-même, était indispensable pour d'autres recherches, restait entièrement inconnue. Or tel est précisément l'objet de la question qu'on s'est proposée dans le Mémoire cité et qu'on y a résolue au moyen d'une analyse dont le principe fondamental consiste à exprimer les propriétés caractéristique du système des formes non-équivalentes répondant à un déterminant quelconque, à l'aide d'une équation dont l'un des membres ne contient rien qui soit relatif à ces formes, tandis que l'autre se compose de suites infinies doubles dont le nombre est égal à celui des formes et dont chacune présente dans son terme général l'une des expressions quadratiques dont il s'agit. L'équation ainsi formée renfermant une variable assujettie à la seule condition de rester supérieure à l'unité, si l'on passe au cas-limite, où cette variable approche indéfiniment de l'unité, les séries doubles tendent toutes vers une limite commune facile a assigner, et l'égalité se transforme de manière à exprimer le nombre des formes par une suite infinie d'une loi très-simple et dont la somme s'obtient aisément avec le secours des formules connues. En effectuant cette dernière opération, on recounait que l'expression du nombre des formes qui répondent à un déterminant quelconque, présente deux cas très-distincts suivant que ce déterminant est un nombre négatif ou positif. Dans le premier de ces cas, l'expression de la loi dont il s'agit a un caractère purement arithmétique, tandis que pour un déterminant positif elle est d'une nature plus composée et en quelque sorte mixte, puisque, outre les éléments arithmétiques dont elle dépend, elle en renferme d'autres qui ont leur origine dans certaines équations auxiliaires qui se présentent dans la théorie des équations binomes, et appartiennent par conséquent à l'Algèbre. Ce dernier résultat est surtout remarquable et offre un nouvel exemple de ces rapports cachés que l'étude approfondie de l'Analyse mathématique nous fail découvrir entre les "questions en apparence les plus disparates.

La solution dont nous venons de rappeler l'idée fondamentale. n'empruntant de la théorie des formes quadratiques que leurs propriétés les plus élémentaires et s'achevant sans difficulté lorsque ces propriétés ont été une fois reconnues et mises en équation, il était naturẹl de chercher à étendre les applications, de ce genre d'analyse et à résoudre par son moyen d'autres questions analogues mais d'un ordre plus élevé. Les questions que l'on doit considérer comme telles, sont assez nombreuses; on peut dans les recherches 
de cette nature, remplacer les formes quadratiques par des fonctions homogènes d'un degré plus élevé, on peut aussi, sans sortir du second degré, et c'est là le cas dont nous nous sommes occupé d'abord et que nous traiterons exclusivement dans ce Mémoire, modifier la nature des formes quadratiques et supposer par exemple que leurs coëfficients sont des entiers complexes. On doit à Mr. Gau/s l'idée de considérer de pareils entiers ${ }^{*}$ ), et l'on sait qu'il y a été conduit par ses recherches sur les résidus biquadratiques, qui lui ont fait reconnaître que la théorie de ces résidus qui parait très-compliquée tant qu'on la rapporte aux entiers réels, se présente sous une face bien différente, lorsqu'on l'envisage sous ce nouveau point de vue, et se résume alors dans une loi de réciprocité d'une simplicité et d'une élégance extrêmes et d'ailleurs parfaitement analogue à celle que l'on connaissait depuis longtemps pour les résidus quadratiques. L'importance de l'idée si profonde que nous venons de rappeler ne consiste pas seulement à amener de pareilles simplifications; elle est d'un usage beaucoup plus étendu et l'on doit la considérer comme ouvrant un nouveau champ aux spéculations arithmétiques.

Avant de transporter dans la théorie des nombres ainsi généralisée la question qui avait été traitée précédemment, il fallait se livrer à un travail préliminaire indispensable et ayant pour objet de se rendre compte des modifications que les propositions fondamentales de la théorie des formes quadratiques doivent subir pour être applicables aux entiers complexes. Ce travail achevé, on a pu reconnaître que les principes dont on avait fait usage dans le Mémoire cité, s'appliquent avec le même succés à la nouvelle question. Seulement, comme cette dernière est d'une nature plus compliquée, les discussions que la solution exige, prennent plus d'étendue et l'on trouve par exemple que pour passer à ce que nous avons nommé plus haut le cas-limite, il faut ici évaluer une intégrale définie quadruple, tandis que précédemment on n'avait eu à considérer que des intégrales doubles, se réduisant d'ailleurs sur le champ à la quadrature de l'ellipse ou de l'hyperbole.

Mais sans entrer ici dans d'autres détails sur la marche de la solution, nous nous bornerons à dire que le résultat définitif est entièrement semblable à celui qui répond au second des deux cas que nous avons

*) Theoria residuorum biquadraticorum. Comment. secunda. 
distingués plus haut. On reconnait en effet que pour un déterminant complexe, le nombre des formes se rattache généralement à la division de la fonction elliptique complète de première espèce dont le module est $\sqrt{\frac{1}{2}}$, ou ce qui revient au même, à la division de la lemniscate en parties égales, le diviseur ou le nombre de ces parties étant un entier complexe.

Outre le résultat dont nous venons d'indiquer la nature, la question présente deux résultats particuliers très-singuliers et tout- $\mathbf{a}$-fait inattendus. Ces résultats sont relatifs aux cas où le déterminant est un entier réel ou le produit d'un tel entier par $\checkmark-1$, le nombre des formes pouvant alors être assigné sans le secours des équations qui se rapportent à la divisiotí des fonctions elliptiques. Pour ne parler ici que du premier de ces deux cas, dont le second ne diffère pas au fond, le résultat consiste en ce que relativement à un entier réel $\boldsymbol{D}$, considéré comme le déterminant de formes quadratiques à coëfficients complexes, le nombre des formes distinetes est égal au produit ou au double produit des deux nombres qui expriment combien il existe de formes pour les deux déterminants opposés $D$ et $-D$, considérés sous le point de vue ordinaire, ces deux cas étant d'ailleurs distingués par un critérium très-simple.

Comme les recherches dont nous venons de présenter l'analyse, exigent des développements assez étendues, nous avons dù diviser notre travail en deux parties, dont la première que nous publions aujourd'hui, contient outre les discussions préliminaires, la solution de la question principale conduite jusqu'au point où elle se trouve dépendre de la sommation d'une série double. Nous terminons cette première partie par l'examen des deux cas particuliers mentionués plus haut, et qui peuvent être traités complètement, sans qu'il soit nécessaire d'effectuer la double sommation. Dans la seconde partie nous acheverons la solution générale et nous discuterons en outre quelques questions accessoires telles que celles qui concernent la distribution des formes quadratiques en genres, et que nous avons dû laisser de côté dans cette première partie, pour ne pas interrompre la marche des considérations qui se rapportent à la question principale.

Quoique les propositions élémentaires de la théorie des entiers complexes aient déjà été exposées par l'illustre géomètre que nous avons cité plus haut, nous avons pensé qu'il pourrait être commode pour le lecteur de trouver dans une courte introduction celles de ces propositions dont nous aurons à faire usage plus tard. 


\section{Définitions et théorèmes préliminaires.}

\section{1.}

On appelle nombre complexe toute expression de la forme $a+b i$, $i$ désignant la quantité imaginaire $r-1$, et $a$ et $b$ ayant des valeurs réellès quelconques. Comme il est souvent nécessaire de distinguer le cas oư l'une des valeurs réelles $a$ et $\boldsymbol{b}$ s'évanouit de celui où ces valeurs sont l'une et l'autre différentes de zéro, nous nommerons l'expression précédente monome ou binome suivant ces deux cas. Le nombre réel et toujours positif, $a^{2}+b^{2}$, le seul cas excepté où l'on a à la fois $a=0, b=0$, sera dit la norme du nombre complexe $a+b i$. Cette norme n'est donc autre chose que ce que l'on appelle communément le quarré du module de l'expression imaginaire $a+b i$. Mais comme ce quarré se présentera beaucoup plus souvent dans nos recherches que le module lui-même, il convient de lui consacrer une dénomination spéciale telle que la précédente déjả proposée par Mr. Gau/s, d'autant plus que l'emploi du mot module pourrait donner lieu à des équivoques. Nous conviendrons de désigner la norme en plaçant la caractéristique $\boldsymbol{N}$ devant le nombre complexe dont il s'agit et d'écrire $N(a+b i)$. Au moyen de ce signe on aura ces équations évidentes et qu'on a souvent occasion d'employer

$$
\boldsymbol{N}(k l)=\mathbf{N}(k) \mathbf{N}(l), \quad \boldsymbol{N}\left(\frac{k}{l}\right)=\frac{\boldsymbol{N}(k)}{\boldsymbol{N}(l)} .
$$

Dans la théorie des nombres complexes on a à considérer ces quatre unités $1, i,-1,-i$, dont l'une quelconque peut être désignée par $i^{\circ}$, en supposant $\rho=0,1,2,3$.

ceux - ci

On appelle nombres complexes associés quatre nombres tels que

$$
a+b i,-b+a i,-a-b i, b-a i,
$$

et dont chacun produit les trois autres, lorsqu'on le multiplie par $i,-1$ et $-i$. Ces quatre nombres sont toujours inégaux à moins qu'on n'ait simultanément $a=0, b=0$.

Deux nombres complexes tels que

$$
a+b i, \quad a-b i
$$

sont dits conjugués, lorsque l'un se change dans l'autre, en remplaçant $i$ par -i. De pareils nombres sont pareillement inégaux excepté lorsque $b=0$. 
Des nombres associés ont toujours une norme commune et la même chose a lieu pour deux nombres conjugués.

Ce qui précède s'applique à des nombres complexes quelconques.

Les nombres complexes $a+b i$ portent differents noms, suivant la nature des nombres réels $a$ et $b$, qu'on en doit considérer comme les éléments. Un nombre complexe $a+b i$ s'appelle entier, lorsque $a$ et $b$ sont l'un et l'autre des entiers, rationnel, lorsque $a$ et $b$ sont l'un et l'autre rationnels, et irrationnel dans tout autre cas. Comme les nombres que nous aurons à considérer, seront presque toujours des nombres complexes entiers, nous supprimerons généralement les adjectifs à moins que cette suppression ne puisse donner lieu à des équivoques.

Lorsque relativement $\dot{a}$ un entier complexe $k$, on a $N(k)=1$, on peut en conclure que $k$ est de la forme $i^{\rho}$. On voit encore par l'équation $\boldsymbol{N}(\boldsymbol{k} l)=\boldsymbol{N}(\boldsymbol{k}) \boldsymbol{N}(\boldsymbol{l})$, que la norme d'un entier $k \boldsymbol{l}$, multiple d'un autre $\boldsymbol{l}$, est elle-même un multiple de celle de ce dernier. Il résulte de là que les diviseurs d'un entier quelconque $m$ ont toujours des normes inférieures ou tout au plus égales à celle de $m$, et que ce dernier cas ne peut avoir lieu que lorsque le diviseur coincide avec le nombre dont il s'agit ou avec l'un de ses trois associés.

Si donc pour abréger on nomme plus grand qu'un autre, un nombre complexe donc la norme surpasse celle de ce dernier, on peut dire que les plus grands diviseurs d'un entier complexe sont cet entier lui-même et ses associés.

Un entier complexe $a+b i$ autre que $i^{\circ}$, est dit composé, lorsqu'il peut se décomposer en deux facteurs qui ne sont ni l'un ni l'autre de la forme $i^{\circ}$. Dans le cas contraire il s'appelle premier.

Il est facile de voir que des nombres associés sont toujours simultanément des nombres premiers ou simultanément des nombres composés, et qu'il en est de même pour deux nombres conjugués.

\section{\$. 2.}

Si $m$ et $m_{1}$ désignent deux entiers complexes quelconques, on pourra toujours trouver un entier complexe $q$ tel que l'on ait $N\left(m-m_{1} g\right) \overline{\overline{2}} \frac{1}{2} N\left(m_{1}\right)$. Il suffit pour s'en assurer, de remarquer qu'on a

$$
\boldsymbol{N}\left(m-m_{1} q\right)=\mathbf{N}\left(m_{1}\right) \mathbf{N}\left(\frac{m}{m_{1}}-q\right),
$$


et que les deux entiers réels qui entrent dans $q$, peuvent toujours être choisis de manière à différer de la partie réelle de $\frac{m}{m_{1}}$ et du coëfficient de $i$ dans cette même expression, de quantités réelles dont les valeurs numériques ne surpassent pas le nombre $\frac{1}{2}$. Il est facile de fonder là-dessus un procédé propre à faire découvrir le plus grand diviseur commun de deux entiers complexes $m$ et $m_{1}$ quelconques. On formera les équations

$$
m=m_{1} q+m_{2}, \quad m_{1}=m_{2} q_{1}+m_{3}, \ldots . m_{h}=m_{h+1} q_{h},
$$

où les entiers $q, q_{1}, \ldots$ sont choisis de manière à ce qu'on ait

$$
\boldsymbol{N}\left(\boldsymbol{m}_{2}\right) \leqq \frac{1}{2} \boldsymbol{N}\left(\boldsymbol{m}_{1}\right), \quad \boldsymbol{N}\left(\boldsymbol{m}_{3}\right) \leqq \frac{1}{2} \cdot \mathbf{N}\left(m_{2}\right), \quad \ldots \cdot 3
$$

ce qui aura nécessairement pour effet de conduire à une dernière équation où $m_{h+2}=0$. Cela fait, il suffit de parcourir les équations précédentes, pour voir que tout diviseur commun de $m$ et $m_{1}$ divise aussi les termes suivants $m_{2}, \ldots$ jusqu'à $m_{h+1}$ inclusiv́ement. Si l'on considère ensuite les mêmes équations en sens inverse, on voit sur le champ que réciproquement tout diviseur de $m_{h+1}$ est aussi diviseur commun de $m$ et $m_{1}$, d'où l'on conclut que le plus grand diviseur commun cherché est l'entier $m_{h+1}$ ou l'un de ses associés, et que dans le cas particulier où $m$ et $m_{1}$ sont premiers entre eux, $m_{h+1}$ sera toujours de la forme $i^{\rho}$. Le procédé précédent conduit facilement à la démonstration du théorème suivant.

„Si, $m$ et $m_{1}$ étant premiers entre eux, le produit $m n$ est divisible „par $m_{1}, n$ sera nécessairement un multiple de $m_{1}$."

En vertu de ce qui précède, on aura nécessairement $m_{h+1}=i^{\circ}$. D'un autre côté, comıne $m n$ est supposé divisible par $m_{1}$, on conclut des équations précédentes multipliées par $n$, que les produits $m_{2} n, m_{3} n, \ldots, m_{h+1} n$ sont également des multiples de $m_{1}$, conclusions dont la dernière coïncide avec le résultat qu'il s'agit d'établir.

Le théorème que nous venons de démontrer, étant entièrement semblable à celui qui dans Ia théorie ordinaire sert de base à toutes les recherches sur les nombres en tant qu'ils sont divisibles les uns par les autres, décomposables en facteurs simples etc., on en tirera les mêmes conséquences pour la théorie des nombres complexes.

En considérant en particulier $m_{1}$ comme un nombre premier absolu, on en conclut qu'un pareil nombre, pour diviser le produit de deux on d'un plus grand nombre de facteurs, doit diviser au moins l'un de ces facteurs De là il suit encore qu'un entier premier à plusieurs autres l'est aussi à 
leur produit, qu'un entier divisible par plusieurs autres qui n'ont pas de diviseur commun, pris deux à deux, l'est aussi par le produit de ces derniers, et ainsi de suite.

Le théorème counu d'après lequel un nombre réel ne peut se décomposer que d'une seule manière en facteurs simples réels, a aussi son analogue dans la théorie des nombres complexes. Il faut seulement, de même que dans le théorème énoncé on considère tacitement les facteurs simples comme positifs ou du moins pris chacun avec un signe déterminé, en agir ici d'une manière analogue. Supposons pour cela que dans chaque groupe de nombres associés, on distingue l'un d'entre eux, d'ailleurs arbitrairement choisi, en l'appelant nombre primaire. Dans cette hypothèse, un entier quelconque $m$ pourra toujours se mettre sous la forme

$$
m=i \rho a b c \ldots \text {, }
$$

$a, b, c$ étant des nombres premiers primaires, égaux on inégaux, et $\mathrm{il}_{\text {est }}$ facile de s'assurer que la décomposition précédente est toujours unique. En effet si on suppose encore

$$
\boldsymbol{m}=i \varphi^{\prime} \boldsymbol{a}^{\prime} \boldsymbol{b}^{\prime} \boldsymbol{c}^{\prime} \ldots .
$$

$a^{\prime}, b^{\prime}, c^{\prime}$ étant pareillement des nombres premiers primaires, il faudra nécessairement pour que ces deux équations s'accordent, que $a$ divise l'un de nombres $a^{\prime}, b^{\prime}, c^{\prime}, \ldots$ Or ces derniers étant premiers et primaires, $a$ devra coïncider avec l'un d'entre eux, avec $a^{\prime}$ par exemple. Divisant les deux équations par $a$ et continuant de procéder toujours de la même manière, l'identité des deux décompositions se trouvera établie. Si, comme on le fait dans la théorie ordinaire, on réunit les facteurs simples égaux sous forme de puissances, on aura douc d'une manière unique

$$
\boldsymbol{m}=i^{\rho} \boldsymbol{a}^{\alpha} \boldsymbol{b}^{\beta} \boldsymbol{c}^{\gamma} \ldots .
$$

$a, b, c, \ldots$ désignant des nombres premiers primaires iuégaux et les exposants $\alpha, \beta, \ldots$ étant tous au moins égaux à l'unité, c'est-à-dire que les bases et leurs exposants, de même que le facteur $i^{\circ}$ seront complètement déterminés dès que le nombre $m$ sera donné.

\$. 3.

Avant d'aller plus loin, il convient de rechercher les conditions propres à faire reconnaitre si un entier complexe est premier ou composé.

I. Considérons d'abord un nombre binome $a+b i$, et soit $\mathbf{N}(a+b i)$ $=p$. Cela posé, il est facile de prouver que $a+b i$ est un nombre pre- 
mier ou non suivant que sa norme, considérée sous le point de vue ordinaire, est elle-même un nombre premier ou composé. Observons d'abord que, si $a+b i$ est composé, en sorte qu'on peut supposer $a+b i=(c+d i)(f+g i)$, $\mathbf{N}(c+d i)>1, \mathbf{N}(f+g i)>1$, on aura $\mathbf{N}(a+b i)=\mathbf{N}(c+d i) \mathbf{N}(f+g i)$, c'est-à-dire égal à un nombre composé. Ce premier point établi, il ne reste évidemment qu'à prouver que si $N(a+b i)=a^{2}+b^{2}$, est un nombre composé, $a+b i$ sera aussi composé, pour que la proposition se trouve démontrée. Suit $a^{2}+b^{2}=m n, m$ et $n$ étant deux entiers réels l'un et l'autre différents de l'unité. Si maintenant $a+b i$, et par suite aussi $a-b i$, était supposé premier, l'équation précédente mise sous la forme $(a+b i)(a-b i)$ $=m n$, exigerait d'après le théorème démontré à la fin du \$. précédent, que $m$ et $n$ fussent également des nombres premiers, sans quoi le second membre renfermerait plus de facteurs simples que le premier, et il faudrait de plus que $m$, abstraction faite d'un facteur de la forme $i^{\circ}$, coïncidât avec l'un des nombres $a+b i, a-b i$, ce qui est impossible, ces derniers étant binomes, tandis que $m$ est monome.

II. D'après ce qu'on vient de prouver, on voit que pour assigner tous les nombres premiers binomes, tout revient à découvrir quels sont parmi les nombres premiers réels et positifs, ceux qui peuvent se décomposer en deux quarrés. Pour ceux de la forme $4 n+3$, une pareille décomposition est impossible, la somme de deux quarrés étant toujours de l'une des formes $4 n$, $4 n+1$, 2. Il ne reste donc que les nombres premiers $4 n+1$, et le nombre 2. Soit $p$ un nombre premier $4 n+1$, il sera facile de prouver, qu'il existe toujours deux groupes de nombres premiers binomes associés ayant $p$ pour norme commune. Cela résulte immédiatement du théorème connu, d'après lequel un nombre premier $4 n+1$ est toujours la somme de deux quarrés, et ne l'est que d'une seule manière. Mais nous n'avons pas besoin de ce théorème, qui peut être considéré au contraire comme un corollaire de la théorie des nombres complexes. Nous supposerons seulement qu'on sache que l'entier réel $\xi$ peut toujours être choisi de manière à rendre la formule $\xi^{2}+1$ divisible par $\mu$, comme cela résulte entr'autres du théorème de Wilson, en vertu duquel on peut poser $\xi=1.2 \ldots \frac{1}{2}(p-1)$. Le produit $(\xi+i)(\xi-i)$ étant ainsi divisible par le nombre $p$, qui ne divise évidemment ni l'un ni l'autre de ces deux facteurs, on en conclut que $p$ est un nombre composé. Soit en conséquence $p=(a+b i)(c+d i), a^{2}+b^{2}>1$, $c^{2}+d^{2}>1$, on aura aussi $p^{2}=\left(a^{2}+b^{2}\right)\left(c^{2}+d^{2}\right)$, d'où l'on conclut, le 
nombre réel $p^{2}$ ne comportant que la seule décomposition $p \times p$ en deux facteurs positifs différents de l'unité, $p=a^{2}+b^{2}=(a+b i)(a-b i)$, où les deux facteurs évidemment binomes, $a+b i, a-b i$, dont la norme commune est un nombre premier réel, seront premiers. Remarquons encore

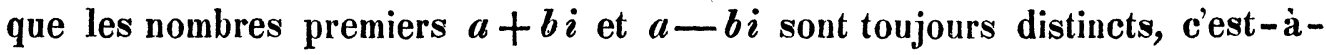
dire qu'ils ne sont ni égaux ni associés. En effet, comme $a, b$ sont évidemment l'un pair, l'autre impair, la supposition $a-b i=i^{\circ}(a+b i)$ exigerait d'abord $i^{\circ}= \pm 1$, et par suite l'une de celles-ci $a=0, b=0$, dont l'impossibilité est manifeste. On voit donc qu'il existe toujours deux groupes distincts de nombres premiers binomes ayant pour norme commune un nombre premier positif $4 n+1$ quelconque, et l'on peut ajouter qu'il n'en existe que deux, car il résulte du théorème déjà cité que, si l'on suppose $\left(a^{\prime}+b^{\prime} i\right)\left(a^{\prime}-b^{\prime} i\right)=(a+b i)(a-b i)$, chacun des facteurs du premier membre est nécessairement égal ou associé à l'un des facteurs du second.

Le nombre 2 qui est également décomposable en deux quarrés, ne donne lieu qu'à un seul groupe de nombres premiers binomes, les deux nombres conjugués $1+i, 1-i$ appartenant pour ce cas au même groupe.

III. Il ne reste qu'à examiner quels sont les nombres monomes qui dans la théorie des entiers complexes jouent le rôle de nombres premiers. Comme sous le rapport dont il s'agit, un nombre quelconque se trouve toujours dans la même catégorie que ses associés, nous n'aurons qu'à considérer des nombres monones positifs, et comme parmi ces derniers, ceux qui sont composés sous le point de vue ordinaire, le sont également dans la théorie des entiers complexes, il n'est plus question que des nombres premiers positifs. Or, les nombres premiers $4 n+1$ et le nombre 2 ayant déjà été reconnus comme nombres composés, il ne reste en définitif qu'à considérer les nombres premiers $4 n+3$, par rapport auxquels il est facile de s'assurer qu'ils sont ici des nombres premiers, comme ils le sont sous -le point de vue ordinaire. En effet, si pour un nombre $q$ de cette espèce on avait $y=(a+b i)(c+d i), N(a+b i)>1, N(c+d i)>1$, et par suite $q^{2}=\left(a^{2}+b^{2}\right)\left(c^{2}+d^{2}\right)$, il faudrait, $q^{2}$ n'étant susceptible que d'une seule décomposition en facteurs positifs différents de l'unité, qu'on eût $q=a^{2}+b^{2}$, ce qui est impossible, comme nous l'avons déjà remarqué.

IV. Les nombres complexes, considérés relativement au diviseur $1+i$, et $\grave{a}$ sa seconde puissance $(1+i)^{2}=2 i$, forment trois classes, pour la désignation desquelles il est utile d'introduire des dénominations spéciales. 
Un nombre sera dit impair, lorsqu'il n'est pas divisible par $1+i$, semi-pair, lorsqu'il est divisible par $1+i$, sans l'être par $(1+i)^{2}$ ou ce qui revient au même, par 2, et pair enfin, lorsqu'il peut être divisé par 2. Il est évident qu'un entier complexe $a+b i$ présentera le premier cas, lorsque les deux entiers réels $a$ et $b$ sont l'un pair, l'autre impair, le second, lorsque ces deux entiers sont l'un et l'autre impairs, et enfin le troisième, lorsque $a$ et $b$ sont tous deux pairs.

V. Nous avons déjà eu occasion de remarquer qu'il peut être utile de distinguer l'un des quatre nombres associés qui forment un même groupe, pour le considérer en quelque sorte comme le nombre primitif ou primaire de ce groupe, les trois autres étant censés dérivés de celui-là en multipliant par $-1, \pm i$. Le besoin d'un telle distinction réglée sur un principe invariable, se fera surtout sentir en tant qu'il s'agira de nombres impairs, et nous conviendrons donc de considérer comme le nombre primaire dans un groupe d'entiers complexes impairs, celui évidemment unique $a+b i$, pour lequel on a simultanément $a \equiv 1(\bmod .4)$, et $b \equiv 0(\bmod .2)$. Il est facile de concluke de cette définition que le produit de deux et par suite d'un nombre quelconque d'entiers impairs primaires, est lui-même un entier primaire. Cette convention embrasse déjà tous les nombres premiers, à l'exception de ceux qui dérivent du nombre 2 , et qui sont $1+i,-1+i$, $-1-i, 1-i$. Quoique relativement à ces derniers, le choix d'un nombre primaire soit peu utile, nous conviendrons pour plus d'uniformilé de regarder comme tel le nombre $1+i$.

\section{S. 4.}

Etant donné un entier complexe quelconque $m$, on peut toujours concevoir la série complète des entiers complexes, distribuée en séries partielles, deux entiers étant rangés dans la même série ou dans des séries distinctes, suivant que leur différence est un multiple de $m$ ou non. Si ensuite on choisit dans chacune de ces séries partielles l'un quelconque des termes qui la composent, on aura ce que nous appelerons un systeme de résidus pour le module donné $m$. Un pareil système jouit donc de la double propriété de contenir un terme et de n'en contenir qu'un, qui soit congru à un entier quelconque suivant le module auquel il répond. Pour construire un système de résidus, tout se réduit à découvrir quelque condition qui soit satisfaite par l'un des termes de toute série partielle et ne 
le soit que par ce seul terme, et $\dot{\mathbf{a}}$ assigner ensuite tous les entiers distincts qui remplissent la condition dont il s'agit. On parviendra, par exemple, à une condition de ce genre, si l'on cherche d'abord, $x+y i$ désignant le terme général d'une série partielle donnée, pour quels termes de cette dernière $y$ a la plus petite valeur non-négative, et si l'on choisit ensuite parmi ces termes en nombre infini, celui nécessairement unique où $x$, supposé pareillement non-négatif, est à son tour un minimum. Pour découvrir la nature d'un pareil terme, soit $m=a+b i$, et désignons par $\alpha+\beta i$ un entier complexe arbitrairement choisi. On aura alors pour le terme général de la série partielle à laquelle cet entier appartient:

$$
x+y i=(a+b i)(t+u i)+\alpha+\beta i, x=a t-b u+\alpha, y=b t+a u+\beta \text {, }
$$
où $t$ et $u$ désignent tous les entiers réels depuis $-\infty$ jusqu'à $\infty$. On voit par la dernière de ces équations, que les valeurs dont $y$ est susceptible, sont toutes telles qu'on a $\gamma \equiv \beta(\bmod . h), h$ désignant le plus grand diviseur commun (positif) de $a$ et $b$. Il résulte de là que $y$ peut toujours ètre égal à l'un des entiers $0,1,2, \ldots ., h-1$, et ne peut l'être qu'à l'un d'entre eux. Soit donc $y_{0}$ celui de ces entiers, qui satisfait à la congruence précédente. Pour que $y$ obtienne cette valeur particulière, on aura à satisfaire à l'équation

$$
\boldsymbol{b} t+\boldsymbol{a u}=y_{0}-\beta,
$$

toujours résoluble et dont la solution complète exprimée en fonction d'une solution particulière $t_{0}, u_{0}$, est

$$
t=t_{0}+\frac{a}{h} z, \quad u=u_{0}-\frac{b}{h} z,
$$

$\approx$ désignant un entier réel arbitraire. Au moyen de ces expressions, l'équation en $x$ deviendra

$$
x=a t_{0}-b u_{0}+\frac{p}{h} z,
$$

où l'on suppose $a^{2}+b^{2}=p$. Comme il reste l'indéterminée $z$ dont nous pouvons disposer à volonté, on voit que la plus petite valeur dont $x$ soit susceptible, est l'une de celles-ci : $0,1,2, \ldots ., \frac{p}{h}-1$, et il est également manifeste que parmi ces dernières il n'en existe qu'une seule que $x$, puisse comporter. Ayant ainsi reconnu que dans toute série partielle il existe toujours un terme unique pour lequel $x$ et $y$ soient resp. compris dans les suites,

$$
x=0,1,2, \ldots ., \frac{p}{h}-1 ; \quad y=0,1,2, \ldots, h-1 ;
$$

on voit que pour obtenir un système de résidus pour le module $a+b i$, on 
n'a qu'à introduire dans l'expression $x+y i$, les valeurs précédentes, combinées de toutes les manières entre elles.

Il $\mathrm{y}$ a un cas particulier qui mérite une attention particulière; c'est celui où $a$ et $b$ sont premiers entre eux, le système à former se réduisant alors simplement à la suite

$$
0,1,2, \ldots, p-1 \text {, }
$$

de sorte que pour un module $m$ de cette nature, on peut toujours satisfaire par un entier réel $\xi$, à la congruence

$$
\xi \equiv k(\bmod m) \text {, }
$$

où $k$ désigne un entier complexe quelconque.

\section{S. 5.}

Le résultat auquel nous venons de parvenir, donne lieu à plusieurs conséquences importantes que nous allons rapidement indiquer.

1. On voit d'abord que le système des résidus, qui répond à un module quelconque $m$, contient toujours un nombre de termes, exprimé par $\boldsymbol{N}(m)$, car on a $\frac{p}{h} h=p=N(m)$.

II. On peut encore assigner séparément combien parmi ces termes il en existe de divisibles par un facteur $k$ de $m$. Il est en effet facile de voir que ces derniers, étant divisés par $k$, constitueront un système de résidus pour le module $\frac{m}{k}$, en sorte que le nombre qu'il s'agit d'obtenir, est exprimé par $\boldsymbol{N}\left(\frac{m}{k}\right)$.

III. Connaissant, par ce qui précède, le nombre des termes dont tout système de résidus pour le module $m$ doit se composer, on peut en conclure que, si l'on a $\boldsymbol{N}(m)$ entiers tels que la différence de deux quelconques d'entre eux, ne soit pas un multiple de $m$, on est dès lors assuré que ces entiers forment un système de résidus relativement au module $m$. Pour faire une application de ce principe, soit $\mu$ le terme général d'un système de résidus pour le module $m$, et désignons par $n$ et $l$ deux entiers déterminés dont le premier n'ait pas de diviseur commun avec $m$. Cela posé, je dis que l'expression $n \mu+l$ représentera également un pareil système. En effet, les valeurs de cette expression étant en nombre convenable, il ne reste plus qu'à s'assurer que deux quelconques d'entre elles ne sauraient présenter une différence multiple de $m$. Or cela est évident, puisque, $\mu^{\prime}$ et $\mu^{\prime \prime}$ désignant deux des valeurs dont $\mu$ est susceptible, la dif- 
férence $n \mu^{\prime}+l-\left(n \mu^{\prime \prime}+l\right)$ est égale au produit $n\left(\mu^{\prime}-\mu^{\prime \prime}\right)$, dont le premier facteur n'a pas de diviseur commun avec $m$, et dont le second n'est pas un multiple de cet entier

L'expression $n \mu+l$ représentant un système de résidus, on voit que parmi les valeurs que $\mu$ comporte, il y a toujours une valeur uuique telle que cette expression soit divisible par $m$, ou en d'autres termes, que la congruence

$$
n x+l \equiv 0 \quad(\bmod . m),
$$

lorsque $n$ n'a pas de diviseur commun avec $m$, est toujours possible, et que sa solution générale est de la forme

$$
x \equiv x_{0} \quad(\bmod . m) \text {, }
$$

$x_{0}$ désignant une solution particulière, de sorte que cette congruence a une racine unique, en considérant à l'ordinaire comme ne constituant qu'une seule racine, toutes les valeurs qui diffèrent les unes des autres de multiples du module. La congruence en question étant équivalente à l'équation

$$
n x+m y+l=0,
$$

on voit encore que la solution générale de celle-ci est donnée par les formules

$$
x \doteq x_{0}+m z, \quad y=y_{0}-n z,
$$

$x_{0}, y_{0}$ désignant une solution particulière quelconque, et $z$ étant un entier complexe arbitraire. Quant à la résolution effective de cette équation ou de là congruence équivalente, elle peut s'effectuer au moyen de l'algorithme employé plus haut pour découvrir le plus grand diviseur commun de deux entiers complexes; mais comme nous n'aurons pas à en faire usage, nous ne nous arrêterons pas sur cette résolution, d'ailleurs entièrenient semblable à celle qui concerne les entiers réels.

IV. Soient maintenant $a, b, c, \ldots$ des entiers complexes en nombre quelconque et premiers entre eux. Construisons des systèmes de résidus pour chacun de ces entiers ainsi que pour leur produit $m=a b c \ldots$. , et désignons par $\alpha, \beta, \gamma, \ldots ; \mu$, les termes généraux de ces systèmes. Cela posé, si relativement à chacun des entiers $\mu$, nous déterminons les nombres $\alpha, \beta, \gamma, \ldots$ qui en diffèrent respectivement d'un multiple de $a, b, c, \ldots$, $\dot{a}$ tout entier $\mu$ se trouvera correspondre une combinaison unique de la forme $\alpha, \beta, \gamma, \ldots$ Prouvons réciproquement que toute combinaison de cette espèce provient toujours de l'un des entiers $\mu$, et ne saurait provenir que de l'un d'entre eux. Cette dernière assertion est facile à justifier; en 
effet si la même combinaison répondait à deux entiers $\mu$ distincts, leur différence serait divisible par $a, b, c, \ldots$ et par suite aussi par $m$, ce qui est contraire à la nature du système dont $\mu$ désigne le terme général. Ayant ainsi reconnu que les combinaisons qui proviennent des entiers $\mu$, sont toutes différentes entre elles, il suffit de remarquer que le nombre de toutes les combinaisons possibles est évidemment $\boldsymbol{N}(\boldsymbol{a}) \boldsymbol{N}(\boldsymbol{b}) \mathbf{N}(\boldsymbol{c}) \ldots$

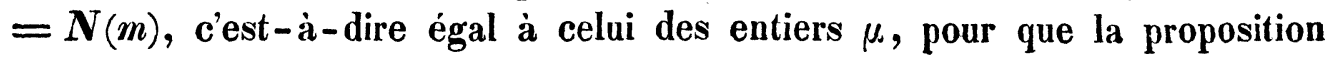
énoncée se trouve établie.

V. Il est facile de voir que, si $\mu$ est premier $\dot{a} m$, les entiers correspondants $\alpha, \beta, \gamma, \ldots$ seront tous respectivement premiers à $a, b, c, \ldots$ et réciproquement. Si donc relativement $\dot{a}$ un entier quelconque $l$, on désigne par $\psi(l)$ le nombre de ceux des termes formant un système de résidus pour le module $l$, qui n'ont pas de diviseur commun avec ce dernier, on aura pour un module $m$, décomposé en facteurs $a, b, c, \ldots$ premiers entre eux, $\psi(m)=\psi(a) \psi(b) \psi(c) \ldots$

VI. Nous pouvons au moyen de la remarque qui vient d'être faite, déterminer la fonction $\psi(m)$ pour un module $m$ quelconque. Soit d'abord $m=a^{\alpha}$, a désignant un nombre premier et l'exposant $\alpha$ étant au moins égal à l'unité. Pour ce cas on obtiendra évidemment le nombre de ceux des termes formant le système des résidus pour le module $m$, qui sont premiers à $m$, si du nombre total des termes du système, on retranche le nombre de ses termes divisibles par $a$. Ces nombres étant le premier

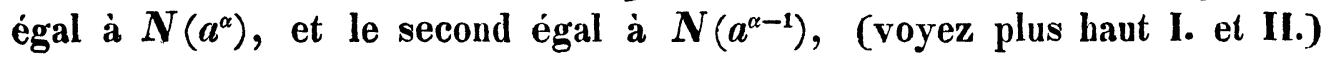
on obtiendra pour la différence cherchée, $\boldsymbol{N}\left(a^{\alpha}\right)-\boldsymbol{N}\left(a^{\alpha-1}\right)=(A-1) A^{\alpha-1}$, en supposant $A=\mathbf{N}(a)$.

Après cela il est facile de voir que relativement à un nombre quelconque

$$
\boldsymbol{m}=i^{\rho} \boldsymbol{a}^{\alpha} \boldsymbol{b}^{\beta} \boldsymbol{c}^{\gamma} \ldots .
$$

$a, b, c$ étant des nombres premiers primaires inégaux, et les exposants $\alpha$, $\beta, \gamma, \ldots$ étant tous différents de zéro, on aura

$$
\psi(m)=(A-1) A^{\alpha-1} \cdot(B-1) B^{\beta-1} \cdot(C-1) C^{\gamma-1} \ldots
$$

en posant pour abréger

$$
\boldsymbol{N}(\boldsymbol{a})=\boldsymbol{A}, \quad \boldsymbol{N}(\boldsymbol{b})=\boldsymbol{B}, \ldots
$$

et l'on doit ajouter que, lorsque $m$ est de la forme $i^{\varrho}$, la fonction $\psi(m)$ se réduit à l'unité positive. 


\section{Théorie des résidus quadratiques.}

\$. 6.

Etant donnés deux entiers complexes quelconques $k$ et $m$, le premier est dit résidu ou non-résidu quadratique par rapport au second, suivant que la congruence $x^{2} \equiv k(\bmod . m)$, dont l'inconnue $x$ est pareillement considérée comme complexe, est ou n'est pas possible. Pour procéder, dans la recherche des conditions propres a distinguer l'un de l'autre ces deux cas, du simple ou composé, nous considérons en premier lieu $m$ comme un nombre premier impair ${ }^{*}$ ) non-diviseur de $k$ qui reste quelconque. Soit

$$
\text { (M) } \quad \mu_{1}, \mu_{2}, \mu_{3}, \ldots
$$

le système des résidus relatif au module $m$, à l'exclusion de celui des termes de ce système, qui est un multiple de $m$. Cela étant, la congruence $\mu x \equiv k(\bmod . m)$, où $\mu$ désigne l'un quelconque des entiers du systême $(M)$, sera toujours satisfaite par une valeur unique $x$ comprise dans la suite $(\boldsymbol{M})$. Distinguons maintenant les deux cas différents que la relation de $k$ à $m$ peut présenter, et supposons d'abord que $k$ soit non-résidu quadratique relativement à $\mu$. Dans cette hypothèse, $x$ sera toujours différent de $\mu$, d'où il suit que les entiers $(\boldsymbol{M})$, peuvent être distribués en groupes composés chacun de deux termes dont le produit soit $\equiv k$ (mod. $m)$. Or, le nombre de ces groupes étant évidemment $\frac{1}{2}(p-1)$, où l'on suppose $p=N(m)$, on aura en multipliant

$$
\left.\mu_{1} \mu_{2} \mu_{3} \ldots \equiv \equiv k^{\frac{1}{(p-1)}} \text { (mod. } m\right) \text {. }
$$

Dans la seconde hypothèse qui est celle de $k$ résidu quadratique par rapport $\dot{a} \cdot \mu$, la distribution en groupes peut encore s'effectuer sur la suite $(\boldsymbol{M})$, après en avoir retranché les termes $\mu$ tels qu'on a $\mu^{2} \equiv k$ (mod.m). Mais comme les termes qui satisfont à cette dernière condition, évidemment toujours au nombre de deux, et tels que l'un est congru à l'autre pris avec le signe moins, donnent un produit $\equiv-k(\bmod . m)$, on voit qu'en multi-

*) Le cas où le module se réduit à la forme $\mathfrak{\imath} \varrho$, ne donne lieu à aucune question, un entier quelconque étant toujours résidu quadratique d'un tel module. Il est néanmoins bón d'observer que les formules qu'on va établir, lorsqu'on y suppose $m$ de cette forme, ne donnent rien d'inexact, pour qu'on soit dispensé dans les recherches générales d'avoir égard à ce cas singulier. 
pliant ce produit par tous les autres termes rangés en groupes, il viendra

$$
\mu_{1} \mu_{2} \mu_{3} \ldots \ldots \equiv-k^{\mathbf{a}^{(p-1)}} \text { (mod. } m \text { ). }
$$

Comme le produit $\mu_{1} \mu_{2} \mu_{3} \ldots$ est indépendant de l'entier $k$, nous pouvons le déterminer en attribuant $\dot{a} k$ une valeur particulière. Si l'on suppose $\dot{a}$ cet effet, $k=1$, ce qui se rapporte évidemment au second cas, on trouve ce résultat

$$
\left.\mu_{1} \mu_{2} \mu_{3} \ldots \equiv \equiv-1 \text { (mod. } m\right)
$$

qui est analogue au théorème connu de Wilson et au moyen duquel les congruences précédentes, réunies en une seule, prennent cette forme plus simple:

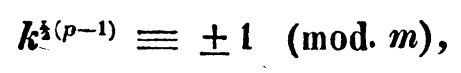

où il faut prendre le signe supérieur ou le signe inférieur, suivant que $k$ est ou n'est pas résidu quadratique relativement $\dot{a} m$. Nous conviendrons de désigner désormais par $\left[\frac{\mathrm{k}}{\mathrm{m}}\right]$ le nombre \pm 1 qui entre dans la congruence précédente, de sorte qu'on aura

$$
k^{\frac{1}{2}(p-1)} \equiv\left[\frac{k}{m}\right](\bmod . m) .
$$

Le symbole $\left[\frac{k}{m}\right]$ est analogue à celui que Legendre a introduit, et dont l'usage est aujourd'hui généralement adopté; mais il importe de ne pas confondre ces deux genres de notations dont la seconde, restreinte aux entiers réels, n'exprime pas toujours la même valeur que celle que nous venons de proposer, désigne pour ce cas particulier. C'est ce qu'on voit par exemple, en supposant $k=2, m=3$, puisqu'on a alors $\left[\frac{2}{3}\right]=1,\left(\frac{2}{3}\right)=-1$. Cette circonsiance n'a d'ailleurs rien qui puisse étonner, une congruence telle que $x^{2} \equiv 2$ (mod. 3$)$, qui n'est pas possible tant que l'inconnue est supposée réelle, pouvant admettre des solutions, lorsque cette inconnue est considérée comme susceptible de valeurs imaginaires.

Relativement à la notation que nous venons d'adopter, on a ces deux équations évidentes:

$$
\text { (a) }\left[\frac{k}{m}\right]=\left[\frac{l}{m}\right], \quad\left[\frac{k}{m}\right]\left[\frac{k^{\prime}}{m}\right]\left[\frac{k^{\prime \prime}}{m}\right] \ldots=\left[\frac{k k^{\prime} k^{\prime \prime} \ldots .}{m}\right],
$$

où l'on suppose $k \equiv l(\bmod . m)$, et où $k, k^{\prime}, k^{\prime \prime}, \ldots$ sont des entiers quelconques non-divisibles par $m$.

Crelle's Journal f. d. M. Bd. XXIV. Heft 4. 
Nous allons maintenant faire voir que la question de savoir si $k$ est ou n'est pas résidu quadratique par rapport $\dot{a} m$, peut toujours se ramener à une question analogue, mais qui ne porte que sur des entiers réels, ou en d'autres termes, nous démontrerons qu'une expression telle que $\left[\frac{k}{m}\right]$ est toujours réductible à une autre de la forme ( ).

Avant de montrer comment cette réduction peut être effectuée, nous remarquerons que, l'expression $\left[\frac{k}{m}\right]$ restant évidemment invariable lorsque le nombre $m$ est remplacé par l'un de ses associés, il sera permis de supposer désormais $m=a+b i$, $a$ et $b$ étant respectivement impair et pair. Cela posé, nous traiterons successivement le cas où $b=0$, et celui où $b$ est différent de zéro.

1. Dans le premier de ces deux cas, on a $m=a$, $a$ désignant, abstraction faite du signe, un nombre premier réel $4 n+3$. Posons de plus $k=\alpha+\beta i$. Pour obtenir la valeur de $\left[\frac{\alpha+\beta i}{a}\right]$, tout se réduit à voir si la congruence

$$
\text { (1) } x^{2} \equiv \alpha+\beta i \quad(\bmod . a)
$$

est ou n'est pas possible. Si l'on y suppose $x=\emptyset+\psi i$, cette congruence se décomposera en ces deux congruences simultanées équivalentes qui ve contiennent que des entiers réels

$$
\text { (2) } \phi^{2}-\psi^{2} \equiv \alpha, \quad 2 \phi \psi \equiv \beta \quad \text { (mod. } a \text { ). }
$$

Ces dernières étant élevées au quarré et ajoutées donnent

$$
\left(\varphi^{2}+\psi^{2}\right)^{2} \equiv \alpha^{2}+\beta^{2}(\bmod . a)
$$

ou ce qui revient au même, $\alpha^{2}+\beta^{2}$ n'étant pas divisible par $a$,

$$
\text { (3) }\left(\frac{\alpha^{2}+\beta^{2}}{a}\right)=1 \text {, }
$$

de sorte que la possibilité de la congruence (1) suppose la condition (3). Je dis réciproquement que, si cette dernière est satisfaite, la possibilité de la congruence (1), ou ce qui revient au même, celle des deux congruences simultanées (2) s'ensuit. Considérons d'abord le cas où $\alpha \equiv 0$ (mod. $a$ ) et dans lequel la condition (3) a évidemment lieu. On voit qu'on satisfait alors à la première des congruences (2), en posant $\psi= \pm \emptyset$, ce qui change la seconde en celle-ci $2 \phi^{2} \equiv \pm \beta(\bmod . a)$, évidemment possible si le signe est convenablement choisi. Reste à considérer le cas où $\alpha$ n'est pas divisible par $a$. En vertu de la condition (3) supposée satisfaite, il existera un entier réel $s$ tel qu'on ait $s^{2} \equiv \alpha^{2}+\beta^{2}$, et par suite $(s+\beta)(s-\beta) \equiv \alpha^{2}(\bmod a)$. 
25. Dirichlet, recherches sur les formes quadratiques.

Or, $\alpha$ n'étant pas divisible par $a$, on conclut de cette dernière congruence,

$$
\left(\frac{s+\beta}{a}\right)=\left(\frac{s-\beta}{a}\right)= \pm 1,
$$

et nous observerons qu'on peut toujours faire en sorte que le signe supérieur ait lieu. En effet la congruence en $s$, d'où nous sommes partis, ne contenant que le quarré $s^{2}$, nous pouvons, lorsque le signe inférieur a lieu, remplacer $s$ par $-s$, ce qui changera les expressions $\left(\frac{s+\beta}{a}\right),\left(\frac{s-\beta}{a}\right)$ respectivement en $-\left(\frac{s-\beta}{a}\right),-\left(\frac{s+\beta}{a}\right)$. On voit donc que, si $s$ est convenablement choisi, on a

$$
\left(\frac{s+\beta}{a}\right)=\left(\frac{s-\beta}{a}\right)=1 .
$$

Cela supposé, on pourra trouver deux entiers réels $t$ et $u$ tels qu'on ait et par suite

$$
t^{2} \equiv s+\beta, \quad u^{2} \equiv s-\beta \quad(\bmod . a) \text {, }
$$

$$
(t u)^{2} \equiv s^{2}-\beta^{2} \equiv \alpha^{2}, \quad t u \equiv \pm \alpha \quad(\bmod . a),
$$

le signe ambigu dépendant du choix de $t$ et $u$. Ajoutons que, les entiers $t$ et $u$ pouvant être pairs ou impairs à volonté, il sera toujours possible de les choisir de même espèce, c'est-à-dire tous les deux pairs ou tous les deux impairs. Cela fait, il est facile de voir qu'on satisfera aux congruences (2) au moyen de ces expressions entières

$$
\varphi=\frac{t \pm u}{2}, \quad \psi=\frac{t \mp u}{2},
$$

où nous supposons que les signes soient choisis conformément à celui qui a lieu dans la congruence $t u \equiv \pm \alpha_{\text {. }}$ C'est ce dont on s'assure sans difficulté, en faisant la substitution et en ayant égard aux conditions auxquelles $s, t, u$ sont supposés satisfaire.

Il résulte de ce qui précède, que, si l'on a $\left[\frac{\alpha+\beta i}{a}\right]=1$, il s'ensuit $\left(\frac{\alpha^{2}+\beta^{2}}{a}\right)=1$, et que la réciproque a également lieu. On conclut de là et de ce que chacune des expressions précédentes est toujours de la forme \pm 1 , que, quel que soit l'entier $\alpha+\beta i$ non-divisible par le nombre premier $a$, on a toujours

$$
\text { (b) } \quad\left[\frac{\alpha+\beta i}{a}\right]=\left(\frac{\alpha^{2}+\beta^{2}}{a}\right) \text {. }
$$

On peut remarquer que dans le cas particulier où l'un des entiers $\alpha, \beta$ s'évanouit, on a $\left[\frac{\alpha+\beta i}{a}\right]=1$. 
II. Considérons maintenant le cas où la partie imaginaire de $m=a+b i$ n'est pas zéro. Pour décider dans ce cas si la congruence $x^{2} \equiv \alpha+\beta i(\bmod m)$, est ou n'est pas possible, nous observerons que d'après ce qui a été prouvé plus haut sur les résidus d'un module tel que $a+b i$, pour lequel $a$ et $b$ sont premiers entre eux, nous pouvons considérer $x$ comme réel. Cela étant, la congruence précédente est équivalente à l'équation $x^{2}-\alpha-\beta i=(\phi+\psi i)(a+b i)$, ou à ces équations simultanées qui ne contiennent que des entiers réels

$$
x^{2}-\alpha=a \phi-b \psi, \quad-\beta=b \varphi+a \psi \text {. }
$$

En les ajoutant, après les avoir multipliées par $a$ et $b$, on trouve

(5) $\quad a x^{2}-a \alpha-b \beta=p \phi$.

Observons maintenant que $a \alpha+b \beta$ ne saurait ètre divisible par $p$. En effet si cela étail, $p$ diviserait aussi $x$, ce qui est impossible en vertu de la congruence $x^{2} \equiv \alpha+\beta i(\bmod . a+b i)$, dont le premier membre est comme le second, premier à $a+b i$ et par conséquent aussi à $p, x$ étant réel. Cela étant, l'équation (5) donne

$$
\left(\frac{a}{p}\right)=\left(\frac{a \alpha+b \beta}{p}\right)
$$

Je dis maintenant que, cette équation qui est une conséquence très-simple de la congrueuce $x^{2} \equiv \alpha+\beta i(\bmod . m)$, étant supposée satisfaite, la possibilité de la congruence ou ce qui revient au même, celle des équations (4), s'ensuit. En effet, la condition (6) entraine immédiatement l'équation (5), qui, en y substituant pour $p$ sa valeur $a^{2}+b^{2}$, se change en

$$
a\left(x^{2}-a-a \phi\right)=b(\beta+b \phi) \text {. }
$$

Or, $a$ et $b$ n'ayant pas de diviseur commun, il faut qu'on ait $\beta+b \varphi=$ $-a \psi, \psi$ étant un entier, et par suite $x^{2}-a-a \varphi=-b \psi$, équations qui coïncident avec celles dont il s'agit de prouver la possibilité.

L'équation (6) pouvant se mettre sous la forme $\left(\frac{a}{p}\right)\left(\frac{a \alpha+b \beta}{p}\right)=1$, on voit que chacune des deux équations

$$
\left[\frac{\alpha+\beta i}{a+b i}\right]=1, \quad\left(\frac{a}{p}\right)\left(\frac{a \alpha+b \beta}{p}\right)=1,
$$

est toujours une conséquence nécessaire de l'autre. De là et de ce que les expressions qui forment leurs premiers membres, sont toujours de la forme \pm 1 , on conclut

$$
\left[\frac{\alpha+\beta i}{a+b i}\right]=\left(\frac{a}{p}\right)\left(\frac{a \alpha+b \beta}{p}\right)
$$


Cette dernière égalité peut prendre une forme plus simple, car on a toujours $\left(\frac{a}{p}\right)=1$. En effet, l'équation $a^{2}+b^{2}=p$, donne sur le champ $\left(\frac{p}{a}\right)=1$, si l'on fait usage du signe de Legendre, étendu, comme l'a proposé Mr. Jacobi, aux nombres composés ${ }^{*}$ ), et par suite, $p$ étant positif et de la forme $4 n+1,\left(\frac{a}{p}\right)=1$. Nous avons donc, quel que soit l'entier $\alpha+\beta i$ nondivisible par le nombre premier $a+b i$, dans lequel $b$ est pair mais différent de zéro:

$$
\text { (c) }\left[\frac{a+\beta i}{a+b i}\right]=\left(\frac{a \alpha+b \beta}{p}\right) \text {. }
$$

Il importe de remarquer que l'équation (b) est tout- $\dot{a}-$ fait distincte de celle que nous venons d'établir, et ne se déduit nullement de cette dernière, en $y$ faisant $b=0$.

\section{\$. 7.}

Nous pouvons maintenant nous occuper de la question que l'on doit regarder comme la plus importante parmi celles que la théorie des résidus quadratiques présente, et qui a pour objet, étant donné un entier complexe quelconque $k$, d'assigner les caractères propres à distinguer les nombres premiers impairs $m$ dont $k$ est résidu quadratique, de ceux auxquels cet entier a la relation opposée. Comme d'après l'équation (a) démontrée plus haut, la question proposée, lorsque $k$ est un nombre composé, se réduit sur le champ $\dot{a}$ des questions analogues relatives aux facteurs de $k$, on voit que nous n'aurons à considérer que les quatre hypothèses,

$$
k= \pm 1, i, \quad 1+i, \alpha+\beta i
$$

$\alpha+\beta i$ étant un nombre premier impair que nous pourrions considérer comme primaire, mais dans lequel nous supposerons simplement que $\beta$, qui peut d'ailleurs s'évanouir, est pair. Le premier cas ne donne lieu à aucune question, \pm 1 étant un quarré. Les trois autres sont résolus par les équations qui suivent et dans lesquelles le nombre premier impair $a+b i$ est pareillement tel que $b$ qui peut d'ailleurs se réduire à zéro, soit pair, et où

*) Les théorèmes qui constituent la théorie des résidus quadratiques, en tant qu'il s'agit de nombres réels, étant généralement connus, nous nous dispenserons d'en rappeler les énoncés, lorsque nous aurons à faire usage de ces théorèmes. Quant à l'usage du signe de Legendre, étendu aux nombres composés, qui est moins connu, on peut sur ce point consulter le compte rendu de l'Académic de Berlin, Oct. 1837, ou le $\$ .2$ du Mémoire déjà cité. R. s. d. a. etc. 
l'on a posé pour abréger, $p=a^{2}+b^{2}$ :

$$
\left[\frac{i}{a+b i}\right]=(-1)^{\frac{p-1}{4}},\left[\frac{1+i}{a+b i}\right]=(-1)^{\frac{(a+b)^{2}-1}{s}},\left[\frac{\alpha+\beta i}{a+b i}\right]=\left[\frac{a+b i}{a+\beta i}\right] .
$$

La première de ces équations se déduit sans difficulté, soit de la formule $k^{\frac{p-1}{2}} \equiv\left[\frac{k}{m}\right]$ (mod. $m$ ) obtenue plus haut, soit des deux équations $(b)$ et $(c)$, si, en suivant cette dernière voie, on suppose successivement $b=0$, et différent de zéro.

Pour démontrer la seconde des équations $(d)$, soit d'abord $b=0$. On a alors au moyen de l'équation $(b)$ et d'un théorème conıu,

$$
\left[\frac{1+i}{a}\right]=\left(\frac{2}{a}\right)=(-1)^{\frac{a^{2}-1}{8}}
$$

conformément à l'équation qu'il s'agit d'établir. Supposons en second lieu, $b$ différent de zéro. L'équation $(c)$ donne alors

$$
\left[\frac{1+i}{a+b i}\right]=\left(\frac{a+b}{p}\right) \text {. }
$$

Pour obtenir la valeur du second membre, nous aurons recours à l'équation identique $2 p=(a+b)^{2}+(a-b)^{2}$, de laquelle on conclut successivement au moyen de théorèmes connus,

$$
\left(\frac{p}{a+b}\right)=\left(\frac{2}{a+b}\right), \quad\left(\frac{a+b}{p}\right)=\left(\frac{2}{a+b}\right)=(-1)^{\frac{(a+b)^{2}-1}{8}},
$$

ce qui s'accorde également avec l'équation que nous nous proposions de vérifier.

La démonstration de la troisième des équations ( $d$ ), à laquelle nous arrivons maintenant et qui exprime une loi de réciprocité entre deux nombres premiers impairs différents, c'est-à-dire ni égaux ni opposés, donne lieu à distinguer trois cas. Le premier de ces cas est celui où $b$ et $\beta$ sont tous les deux égaux à zéro. Dans ce premier cas, la vérité de l'équation est évidente, puisque d'après la formule (b) on a à la fois $\left[\frac{\alpha}{a}\right]=1,\left[\frac{a}{\alpha}\right]=1$. Considérons en second lieu, le cas où l'un des entiers $b$ et $\beta$ se réduit à zéro, et soit $\beta$ cet entier évanouissant, ce que la forme symétrique de notre équation permet évidemment de supposer. On a alors en vertu des équations $(c)$ et $(b)$ et d'après une remarque déjà faite,

$$
\left[\frac{\alpha}{a+b i}\right]=\left(\frac{a \alpha}{p}\right)=\left(\frac{\alpha}{p}\right), \quad\left[\frac{a+b i}{\alpha}\right]=\left(\frac{p}{\alpha}\right),
$$


de sorte que la vérification à effectuer résulte de l'équation connue

$$
\left(\frac{\alpha}{p}\right)=\left(\frac{p}{\alpha}\right) \text {. }
$$

Passant enfin au troisième cas où $b$ et $\beta$ sont l'un et l'autre différents de zéro, on appliquera la formule $(c)$ à chacun des deux membres de l'équation qu'il s'agit de prouver et qui deviendra ainsi

$$
\left(\frac{a \alpha+b \beta}{p}\right)=\left(\frac{a \alpha+b \beta}{\varpi}\right)
$$

en posant pour un instant, $\alpha^{2}+\beta^{2}=\varpi$. Pour s'assurer de la vérité de cette dernière, il suffit de recourir à l'équation identique $(a \alpha+b \beta)^{2}+(b \alpha-a \beta)^{2}$ $=p_{\varpi}$, d'où il suit successivement, $a \alpha+b \beta$ étant impair,

$$
\left(\frac{p}{a \alpha+b \beta}\right)=\left(\frac{\varpi}{a \alpha+b \beta}\right), \quad\left(\frac{a \alpha+b \beta}{p}\right)=\left(\frac{a \alpha+b \beta}{\varpi}\right) \text { c. q. f. p. }
$$

Nous ne terminerons pas ce $\$$. sans observer que les équations $(d)$ sont dues à Mr. Gaufs qui les a données sans démonstration, du moins la dernière, dans le Mémoire cilé plus haut. La démonstration que nous venons de développer et qui avait déjà été indiquée dans un Note insérée dans ce Journal, est, comme on voit, une application très-simple des théorèmes $(b)$ et $(c)$, qui indépendamment de l'usage que nous en faisons ici, nous seront indispensables pour la solution de la question qui fait le principal sujet du présent Mémoire.

\section{§. 8.}

Le symbole $\left[\frac{k}{m}\right]$, tel que nous l'avons employé jusqu'à présent, suppose que $m$ est un nombre premier impair. Il arrive souvent qu'on a à considérer des produits de la forme

$$
\left[\frac{k}{m}\right]\left[\frac{k}{m^{\prime}}\right]\left[\frac{k}{m^{\prime \prime}}\right] \ldots,
$$

où $m, m^{\prime}, m^{\prime \prime}, \ldots$ sont des nombres premiers impairs non-diviseurs de $k$, mais d'ailleurs égaux ou inégaux. Soit $M=m m^{\prime} m^{\prime \prime} \ldots$ et convenons de désigner désormais le produit précédent simplement par

$$
\left[\frac{k}{M}\right]
$$

de sorte que la valeur de notre symbole ainsi généralisé, toujours égale soit $\dot{a}+1$ soit $\dot{a}-1$, n'indiquera plus suivant ces deux cas, si $k$ est ou n'est pas résidu quadratique par rapport $\dot{a} \boldsymbol{M}$, et fera seulement connaitre, si parmi les facteurs simples égaux ou inégaux de $M$, il y en a un nombre 
pair ou impair, anxquels $k$ présente la dernière de ces deux relations. L'extension que nous venons d'indiquer et qui est entièrement semblable à celle que Mr. Jacobi a proposée relativement au signe de Legendre et dont nous avons déjà fait un fréquent usage dans ce qui précède, donne lieu à plusieurs théorèmes analogues à ceux démontrés dans le $\$$. précédent et faciles à déduire de ces derniers. On a d'abord évidemment

(e) $\left[\frac{k}{M}\right]=\left[\frac{l}{M}\right], \quad\left[\frac{k k^{\prime}}{M}\right]=\left[\frac{k}{M}\right]\left[\frac{k^{\prime}}{M}\right], \quad\left[\begin{array}{c}k \\ M M^{\prime}\end{array}\right]=\left[\frac{k}{M}\right]\left[\frac{k}{M^{\prime}}\right]$,

équations qui supposent, la première, que $k$ toujours sans diviseur commun avec l'entier impair $M$, est tel qu'on ait $k \equiv l(\bmod . M)$, la seconde que $k$ et $k^{\prime}$ sont premiers à $M$, et enfin la troisième que $k$ est premier aux entiers impairs $M$ et $M^{\prime}$.

Voici maintenant les équations analogues aux équations $(d)$, ou pour mieux dire, d'une forme toute identique avec ces dernières:
(f)
$\left[\frac{i}{A+B i}\right]=($
1) $\frac{P-1}{4}$
$\left[\frac{1+i}{A+B i}\right]$
1) $\frac{(A+B)^{2}-1}{8}$
$\left[\frac{\alpha+\beta i}{A+B i}\right]=\left\lfloor\frac{A+B i}{\alpha+\beta i}\right]$

Dans ces équations $A+B i$ et $\alpha+\beta i$ sont deux entiers complexes impairs quelconques premiers entre eux et pour lesquels les coëfficients $B$ et $\beta$, toujours considérés comme pairs, peuvent se réduire à zéro. On a d'ailleurs $\boldsymbol{P}=\boldsymbol{A}^{2}+\boldsymbol{B}^{2}$.

La première de ces équations cö̈ncidant avec la première des équations $(d)$ déjà établies, lorsque $A+B i$ se réduit au nombre premier $a+b i$, on voit que pour s'assurer qu'elle a généralement lieu, tout se réduit $\dot{a}$ faire voir que, si on la suppose exacte pour un nombre quelconque $\boldsymbol{A}+\boldsymbol{B} i$, elle ne cessera pas de subsister lorsque ce dernier vient à être remplacé par le produit $(a+b i)(A+B i)=A^{\prime}+B^{\prime} i$. Nous avons donc a faire voir que la troisième des équations

$$
\left[\frac{i}{a+b i}\right]=(-1)^{\frac{p-1}{4}},\left[\frac{i}{A+B i}\right]=(-1)^{\frac{p-1}{4}},\left[\frac{i}{A^{\prime}+B^{\prime} i}\right]=(-1)^{\frac{p^{\prime}-1}{4}},
$$

où l'on suppose $p=a^{2}+b^{2}, P^{\prime}=A^{\prime 2}+B^{\prime 2}$, est une conséquence des deux premières. Il suffit évidemment pour cela, de prouver que les deux entiers réels

$$
\frac{p-1}{4}+\frac{p-1}{4}, \frac{p^{\prime}-1}{4}
$$

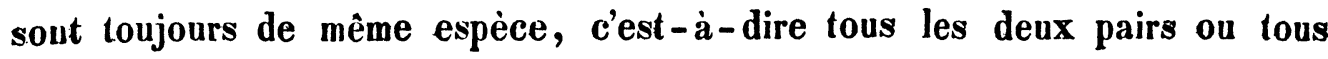
les deux impairs. C'est ce qui résulte sur le champ de l'équation identique 
25. Dirichlet, recherches sur les formes quadratiques.

$$
\frac{P p-1}{4}-\frac{p-1}{4}-\frac{P-1}{4}=\frac{(P-1)}{4} \underline{(p-1)}
$$

dont le second membre est pair et même divisible par $4, \boldsymbol{P}$ et $\boldsymbol{p}$ étant de la forme $4 n+1$.

Le même moyen de démonstration peut s'appliquer à la seconde des équations $(f)$, et l'on voit qu'il s'agira ainsi de démontrer que les deux nombres

$$
\frac{(a+b)^{2}-1}{8}+\frac{(A+B)^{2}-1}{8}, \frac{\left(A^{\prime}+B^{\prime}\right)^{2}-1}{8}
$$

sont toujours de même espèce. Observons pour cela qu'en vertu de l'équation identique

$$
\frac{(r s)^{2}-1}{8}-\frac{r^{2}-1}{8}-\frac{s^{2}-1}{8}=\frac{\left(r^{2}-1\right)\left(s^{2}-1\right)}{8},
$$

dont le second membre est pair, si $r$ et $s$ sont des entiers impairs, le premier des deux entiers que nous avons à considérer, est de même espèce que $\frac{((a+b)(A+B))^{2}-1}{8}$. Mais, comme d'un autre côté deux quarrés dont les racines diffèrent d'un multiple de $\delta$, diffèrent eux-mêmes d'un multiple de 16, ce dernier est à son tour de même espèce que

$$
\frac{((a+b)(A+B)-2 B b)^{2}-1}{8}=\frac{\left(A^{\prime}+B^{\prime}\right)^{2}-1}{8},
$$

et l'assertion avancée se trouve établie.

La troisième des équations $(f)$ est également très-facile à établir. En effet, d'après l'hypothèse faite sur les entiers $A+B i, \alpha+\beta i$, ils peuvent se décomposer l'un et l'autre en facteurs simples ayant leurs parties réelles impaires. Soit $n$ l'un des facteurs de $\boldsymbol{A}+\boldsymbol{B} \boldsymbol{i}$, et $\boldsymbol{m}$ l'un de ceux de $\alpha+\beta \boldsymbol{i}$, on pourra par l'emploi répété des deux dernières des équations $(e)$, remplacer l'expression $\left[\frac{\alpha+\beta i}{A+\overline{B i}}\right]$ par un produit d'expressions de la forme $\left[\frac{m}{n}\right]$, où tout facteur $m$ doit être combiné avec tout facteur $n$. Or, si maintenant on remplace tout symbole $\left[\frac{m}{n}\right]$ par celui -ci $\left[\frac{n}{m}\right]$ qui lui est équivalent en vertu de la troisième des équations $(d)$, et que l'on effectue la multiplication au moyen des équations déjà citées, le premier membre de l'équation qu'il s'agit de vérifier, se trouvera identique au secoud.

Il reste à opérer d'une manière générale la réduction qui pour le cas particulier d'un nombre premier $a+b i$, peut s'obtenir au moyen des Crelle's Journal f. d. M. Bd. XXIV. Heft 4. 
équations $(b)$ et $(c)$ du $\$$. précédent. Soit $\boldsymbol{A}+\boldsymbol{B} i$ un entier impair quelconque ( $\boldsymbol{B}$ étant pair et pouvant se réduire a zéro) et $\alpha+\beta i$ un second entier assujetti à la condition unique d'être premier à $\boldsymbol{A}+\boldsymbol{B} i$; il s'agira de remplacer l'expression $\left[\frac{\alpha+\beta i}{A+B i}\right]$ par des expressions analogues ne contenant que des entiers réels. Considérons d'abord le cas où $\boldsymbol{B}$ s'évanouit, et celui où $\boldsymbol{A}$ et $\boldsymbol{B}$ n'ont pas de diviseur commun; nous verrons ensuite que le cas le plus général se réduit immédiatement à ceux - là. Relativenient aux deux cas qui viennent d'être indiqués, on a respectivement

$$
\text { (g) }\left[\frac{\alpha+\beta i}{A}\right]=\left(\frac{\alpha^{2}+\beta^{2}}{A}\right),\left[\frac{\alpha+\beta i}{A+B i}\right]=\left(\frac{A \alpha+B \beta}{P}\right) \text {, }
$$

$\boldsymbol{P}$ désignant pour abréger dans la seconde de ces équations, le binome $\boldsymbol{A}^{2}+\boldsymbol{B}^{2}$. Pour démontrer la première, observons qu'on peut y considérer $\boldsymbol{A}$ comme positif, les deux membres ne changeant pas lorsqu'on y remplace $A$ par $-\boldsymbol{A}$. Cela posé, soit

$$
\boldsymbol{A}=\mathbf{a a}^{\prime} \ldots . . \times \boldsymbol{p p}^{\prime} \ldots .,
$$

a, $a^{\prime}, \ldots ; p, p^{\prime}, \ldots$ désignant des nombres premiers réels et positifs, les premiers de la forme $4 n+3$, les seconds de la forme $4 n+1$.

D'après l'équation $(b)$, chacun des premiers donne une équation telle que

$$
\left[\frac{\alpha+\beta i}{\mathrm{a}}\right]=\left(\frac{\alpha^{2}+\beta^{2}}{\mathrm{a}}\right),
$$

tandis que pour chacun des derniers, $p$ par exemple, qui peut se décomposer en deux facteurs premiers binomes $(a+b i)(a-b i)$, où $b$ est supposé pair, on a en vertu de l'équation $(c)$,

$\left[\frac{\alpha+\beta i}{p}\right]=\left[\frac{\alpha+\beta i}{a+b i}\right]\left[\frac{\alpha+\beta i}{a-b i}\right]=\left(\frac{a \alpha+b \beta}{p}\right)\left(\frac{a \alpha-b \beta}{p}\right)=\left(\frac{a^{2} \alpha^{2}-b^{2} \beta^{2}}{p}\right)$, et par suite, puisque $-b^{2} \equiv a^{2}(\bmod . p)$,

$$
\left[\frac{\alpha+\beta i}{p}\right]=\left(\frac{a^{2}}{p}\right)\left(\frac{\alpha^{2}+\beta^{2}}{p}\right)=\left(\frac{a^{2}+\beta^{2}}{p}\right) \text {. }
$$

Ces deux systèmes d'équations étant multipliés entre eux, donnent la formule qu'il s'agissait d'établir.

Passons à la vérification de la seconde des équations précédentes.

Nous supposerons d'abord $\beta=0$, cas auquel celui où $\beta$ n'est pas zéro, se ramène facilement. Comme par hypothèse, $A$ et $B$ n'ont pas de diviseur 
commun, on pourra poser

$$
\boldsymbol{A}+\boldsymbol{B} i=(a+b i)\left(a^{\prime}+b^{\prime} i\right) \ldots .
$$

où les facteurs du second membre désignent des nombres premiers binomes, dans lesquels $b, b^{\prime}, \ldots$ sont supposés pairs. L'équation $(c)$ donne relativement au facteur $a+b i$,

$$
\left[\frac{\alpha}{a+b i}\right]=\left(\frac{a \alpha}{p}\right)=\left(\frac{\alpha}{p}\right)
$$

en posant pour abréger $a^{2}+b^{2}=p$. En faisant le produit de cette équa tion et des équations analogues, il viendra

$$
\left[\frac{\alpha}{A+B i}\right]=\left(\frac{\alpha}{p p^{\prime} \ldots . .}\right)=\left(\begin{array}{l}
\alpha \\
\bar{P}
\end{array}\right) \text {. }
$$

Or, l'équation qu'il s'agit de prouver se réduisant par la supposition $\beta=0$, $\dot{a}$ celle-ci

$$
\left[-\frac{\alpha}{A+B i}\right]=\left(\frac{A \alpha}{P}\right)=\left(\frac{A}{P}\right)\left(\frac{\alpha}{P}\right),
$$

nous n'avons plus qu'à démontrer qu'on a $\left(\frac{A}{P}\right)=1$. Mais, comme $A$ et $\boldsymbol{B}$ sont premiers entre eux, il résulte de l'équation $\boldsymbol{A}^{2}+\boldsymbol{B}^{2}=\boldsymbol{P}$, que $\boldsymbol{A}$ et $\boldsymbol{P}$ sont pareillement sans diviseur commun, de sorte que $\left(\frac{P}{A}\right)=1$, et par suite $\left(\frac{A}{P}\right)=1$.

Reste à considérer le cas où $\beta$ a une valeur différente de zéro. On cherchera alors un entier réel $s$ tel qu'on ait

$$
s \equiv \alpha+\beta i(\bmod . \boldsymbol{A}+\boldsymbol{B} i),
$$

dont l'existence suit de l'hypothèse admise sur les nombres $\boldsymbol{A}$ et $\boldsymbol{B}$. Cela fait, on aura

$$
\left[\frac{\alpha+\beta i}{A+B i}\right]=\left[\frac{s}{A+B i}\right]
$$

et par suite, en vertu du cas déjả démontré,

$$
\left[\frac{\alpha+\beta i}{A+B i}\right]=\left(\frac{A s}{\bar{P}}\right) \text {. }
$$

D'un autre côté, si l'on remplace la congruence précédente par deux équations équivalentes, on reconnait sur le champ que $s$ satisfait à la condition $\boldsymbol{A} s \equiv \boldsymbol{A} \alpha+\boldsymbol{B} \beta(\bmod \boldsymbol{P})$.

Cela étant, cette dernière congruence donne l'équation

$$
\left(\frac{A s}{P}\right)=\left(\frac{\boldsymbol{A} \alpha+\boldsymbol{B} \beta}{\boldsymbol{P}}\right)
$$

dont la comparaison avec celle obtenue plus haut, donne un résultat qui 
s'accorde avec la seconde des formules $(g)$. Il nous reste enfin $\dot{a}$ sup-

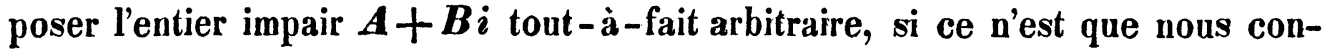
sidérons toujours $B$ comme pair, ce qui ne nuit en rien à la généralité. Soit $L$ le plus grand diviseur commun (réel) de $\boldsymbol{A}$ et $\boldsymbol{B}$, et posons $\boldsymbol{A}=$ $A^{\prime} L, \quad B=B^{\prime} L, \quad A^{\prime 2}+B^{\prime 2}=P^{\prime}$. L'expression $\left[\frac{\alpha+\beta i}{A+B i}\right]$ dans laquelle $\alpha+\beta i$ n'est assujetti qu'à la seule condition d'être premier à $A+B i$, se décomposera alors dans les deux facteurs

$$
\left[\frac{\alpha+\beta i}{L^{2}}\right]\left[\frac{\alpha+\beta i}{A^{\prime}+\bar{B}^{\prime} i}\right]
$$

respectivement de même forme que les premiers membres des équations $(g)$, et l'on aura en conséquence:

$$
\text { (h) }\left[\frac{\alpha+\beta i}{A+B i}\right]=\left(\frac{\alpha^{2}+\beta^{2}}{L}\right)\left(\frac{A^{\prime} \alpha+B^{\prime} \beta}{\boldsymbol{P}^{\prime}}\right) \text {. }
$$

§. 9.

Nous terminerons ce que nous avons à dire sur les résidus quadratiques, en considérant la congruence

$$
\text { (1) } x^{2} \equiv k(\bmod . m) \text {, }
$$

où $k$ et $m$ sont des entiers complexes quelconques premiers entre eux et le second de plus impair. Pour que cette congruence soit possible, il faut évidemment qu'elle puisse subsister par rapport à chacun des facteurs simples de m. Soient

$$
f, f^{\prime \prime}, f^{\prime \prime}, \ldots
$$

les nombres premiers primaires inégaux qui divisent $m$ et soit $\mu$ leur nombre. 11 faudra donc qu'on ait

$$
\text { (2) }\left[\frac{k}{f}\right]=1,\left[\frac{k}{f^{\prime}}\right]=1,\left[\frac{k}{f^{\prime \prime}}\right]=1, \ldots
$$

Je dis de plus que, ces conditions ayant lieu, la possibilité de la congruence s'ensuit et que le nombre de ses racines sera $2^{\mu}$, en considérant à l'ordinaire comme ne constituant qu'une seule racine, les entiers en nombre infini qui différent les uns des autres de multiples du module $m$. Considérons d'abord la congruence $x^{2} \equiv k\left(\bmod . f^{h}\right)$, l'exposant étant un nombre positif quelconque. Si l'on y satisfait par la supposition $x=a$, et par suite par l'hypothèse plus générale $x=\alpha+t f^{h}, t$ étant un entier arbitraire, il est facile d'en déduire une solution pour la congraence de même forme, mais relative au module $f^{h+l}$ où l'on suppose $l \leqq h$. En effet, 
la substitution de l'expression de $x$ donnant

$$
\frac{x^{2}-k}{f^{h}}=\frac{\alpha^{2}-k}{f^{h}}+2 \alpha t+t^{2} f^{h}
$$

où le premier terme du second nembre est par hypothèse un entier, on voit que pour satisfaire $\dot{a}$ la congruence $x^{2} \equiv k$ (mod. $f^{h+l}$ ), il reste $\dot{a}$ faire en sorte qu'on ait $2 \alpha t \equiv-\frac{\alpha^{2}-k}{f^{h}}\left(\bmod . f^{l}\right)$, ce qui est toujours possible, $\alpha$ et par suite $2 \alpha$ étant évidemment premier au module. Comme on peut par ce procédé, s'élever à des exposants de plus en plus grands, en partant de l'exposant $l=1$, on voit que la condition de possibilité de la congruence

$$
x^{2} \equiv k \quad\left(\bmod . f^{h}\right),
$$

quel que soit $h$, est la même que celle qui se rapporte à $h=1$, et qui consiste en ce que l'on doit avoir $\left[\frac{k}{f}\right]=1$. Voyons maintenant quel est le nombre des racines de la congruence précédente. En considérant toujours a comme une de ses racines, on pourra lui donner la forme

$$
x^{2}-\alpha^{2}=(x+\alpha)(x-\alpha) \equiv 0 \quad\left(\text { mod. } f^{h}\right) \text {. }
$$

Or, $x+a$ et $x-\alpha$ ne pouvant être simultanément divisibles par $f$, on voit qu'on ne peut satisfaire à cette dernière qu'en supposant

$$
x \equiv \alpha \quad \text { ou } \quad x \equiv-\alpha \quad\left(\bmod . f^{h}\right)
$$

ce qui ne donne que deux racines, qui seront toujours distinctes, leur différence $2 \alpha$ n'étant pas divisible par $f$.

Si maintenant l'on observe que, si l'on pose $m=i^{\circ} f^{h} f^{h^{\prime}} \ldots$, la congruence (1) est évidemment équivalente à ces congruences simultanées

$$
\left.x \equiv k \quad \text { (mod. } f^{h}\right), \quad x^{2} \equiv k \quad\left(\bmod . f^{\prime h^{\prime}}\right), \quad \ldots \ldots
$$

dont chacune, en vertu de ce qui précède, admet deux racines distinctes de la forme $\pm \alpha$, on conclura facilement d'après la remarque faite plus haut $\$ .5, I V$, que la congruence (1) admet elle-mème $2^{\mu}$ racines distinctes, lorsque les conditions (2), nécessaires pour sa possibilité, sont toutes remplies. II est bon d'ajouter que dans le cas oú $m$ est de la forme $i^{\varrho}$, et où il n'y a aucune condition à remplir, le nombre des solutions de la congruence (1) est toujours exprimé par la formule $2^{\mu}$, car on a dans ce cas, $\mu=0$. 


\section{Théorèmes fondamentaux sur les formes quadratiques.}

\section{\$. 10.}

Avant d'entrer dans le sujet indiqué par le titre, il convient de faire uwe remarque nécessaire pour que l'exposition qu'on va lire, soit considérée sous son véritable point de vue. L'objet du présent mémoire étant purement théorique, nous avons cherché à résoudre les questions que nous avions a traiter, par les considérations qui, théoriquement parlant, nous ont paru les plus simples, sans nous attacher à rendre les solutions qui en dérivent, propres au calcul numérique. Pour satisfaire à cette dernière condition, il faudrait entrer dans des développements assez étendus, qui ne présenteraient que très-peu d'intérêt et ne seraient d'ailleurs d'aucune utilité pour l'objet que nous avons en vue et qui, comme nous l'avons déjà dit, est de pure théorie. Limitès comme nous venons de l'indiquer, les éléments de la théorie des formes quadratiques $\dot{a}$ coëfficients et à indéterminées complexes, peuvent être présentés dans un petit nombre de pages, si aux moyens déjà employés par les illustres géomètres qui ont fondé ou perfectionné la théorie analogue relative aux entiers réels, on ajoute quelques principes nouveaux, qui nous paraissent mériter l'attention des géomètres par leur extrême fécondité qui ne sera toutefois mise dans tout son jour que par des recherches ultérieures que nous avons entreprises sur les formes des degrés supérieurs et que nous aurons $\dot{a}$ exposer plus tard.

Toute expression telle que

$$
\text { (1) } a x^{2}+2 b x y+c y^{2}
$$

où $a, b, c$ sont des entiers complexes déterminés, et $x, y$ de pareils entiers indéterminés, est ce que nous appellerons une forme guadratique binaire ou simplement une forme, cette abréviation ne pouvant donner lieu ici à aucune ambiguité. Il est essentiel de suivre un ordre fixe tant par rapport aux indéterminées $x$ et $y$, qui seront respectivement nommées la première et la seconde, que par rapport aux coëfficients $a, b, c$, dont la désignation indiquera toujours la place que ces coëfficients occupent dans l'expression que nous venons d'écrire.

Les propriétés de la forme (1), dépendant principalement du nombre $D$, donné par l'équation $D=b^{2}-a c$, ce nombre sera dit le déterminant de la forme en question. Dans le cas particulier où $D$ est un quarré, ce 
qui comprend la supposition de $D=0$, la forme se décompose évidemment en deux facteurs linéaires à coëfficients rationnels, en sorte que ses propriétés se déduisent facilement de celles bien comnues des expressions de ce genre. C'est pourquoi nous ferons toujours abstraction de ce cas particulier. Sous cette restriction, les coëfficients extrêmes $a$ et $c$ sont l'un et l'autre différents de zéro, d'où il suit que l'un d'entre eux, $c$ par exemple, peut se déduire sans indétermination de l'autre $a$, du coëfficient moyen $b$ et du déterminant $D$, supposés connus, au moyen de la formule $c=\frac{b^{2}-D}{a}$.

Si dans la forme (1) on remplace les indéterminés $x$ et $y$, par de nouvelles indéterminées $x^{\prime}$ et $y^{\prime}$, liées aux premières par les équations

$$
\text { (2) } x=a x^{\prime}+\beta y^{\prime}, \quad y=\gamma x^{\prime}+\delta y^{\prime},
$$

où $\alpha, \beta, \gamma, \delta$ sont des entiers donnés, elle se changera en cette autre

où l'on a

$$
\text { (3) } a^{\prime} x^{\prime 2}+2 b^{\prime} x^{\prime} y^{\prime}+c^{\prime} y^{\prime 2}
$$

$$
\begin{gathered}
\text { (4) } a^{\prime}=a \alpha^{2}+2 b a \gamma+c \gamma^{2}, \quad b^{\prime}=a a \beta+b(\alpha \delta+\beta \gamma)+c \gamma \delta, \\
c^{\prime}=a \beta^{2}+2 b \beta \delta+c \delta^{\prime 2},
\end{gathered}
$$

et l'on dit alors que la nouvelle forme (3) est contenue sous la forme primitive (1). En substituant les coëfficients $a^{\prime}, b^{\prime}, c^{\prime}$ de la forme (3), dans l'expression de son déterminant $\boldsymbol{D}^{\prime}$, il viendra

$$
\text { (5) } \quad \boldsymbol{D}^{\prime}=(\alpha \delta-\beta \gamma)^{2} \boldsymbol{D} \text {. }
$$

On voit ainsi qu'une forme contenue sous une autre, a toujours un déterminant multiple de celui de cette dernière, et que le quotient de ces déterminants est un quarré, d'où il suit que, pour que deux formes puissent se contenir mutuellement, il faut nécessairement que leurs déterminants soient ou égaux ou opposés. Réciproquement, si les déterminants de deux formes telles que (1) et (3), sont égaux ou opposés, et qu'en outre la première contienne la seconde, je dis que celle-ci contiendra la première. Pour le prouver, remarquons que l'hypothèse $D^{\prime}= \pm D$, comparée à l'équation (5), donne celle-ci

$$
\text { (6) } \quad \alpha \delta-\beta \gamma=i^{i}
$$

les déterminauts éganx a zéro étant toujours exclus. Si maintenant l'on résout les équations (2) par rapport $\dot{\mathfrak{a}} x^{\prime}$ et $y^{\prime}$, on obtiendra les expressions

$$
\text { (7) } x^{\prime}=\frac{\delta}{i^{\rho}} x-\frac{\beta}{i^{i}} y, \quad y^{\prime}=-\frac{\gamma}{i^{q}} x+\frac{\alpha}{i^{i}} y,
$$

dont les quatre coëfficients sont entiers, et qui, étant introduites dans la 
forme (3), la feront évidemment coïncider avec la forme (1), ce qu'il s'agissait de prouver.

Deux formes dont chacune contient l'autre, sont dites éyuivalentes. Quoique la relation mutuelle de deux formes, exprimée par cette désignation, puisse subsister aussi bien entre deux formes à déterminants opposés qu'entre deux formes dont les déterminants sont égaux, nous nous bolnerons à considérer le deruier de ces deux cas. Il est en effet facile de voir que ces deux cas ne sont pas essentiellement différents, puisque, étant données deux formes qui répondent au premier, il suffit évidemment de multiplier les trois coëfficients de l'une d'entre elles respectivement par $1, i,-1$, pour que le groupe des deux formes rentre dans le second de ces deux cas.

La définition de l'équivalence ainsi restreinte, donne encore lieu à une nouvelle subdivision qu'il est essentiel de prendre en considération. Comme on a $D^{\prime}=D$, et par suite en vertu de l'équation (5),

(8) $\alpha \delta-\beta \gamma= \pm 1=\varepsilon$,

nous pouvons avoir égard au signe dont l'unité est précédée dans cette équation; nous dirons désormais que la substitution donnée par les formules (2), et qui change la forme (1) dans la forme équivalente (3), est propre ou impropre, suivant que le signe supérieur ou le signe inférieur a lieu dans l'équation (8). Observons d'abord que la substitution inverse (7) qui sert à revenir de la forme (3) à la forme (1), et qui pour le cas qui nous occupe, se réduit à poser

$$
x^{\prime}=\frac{\delta}{\varepsilon} x-\frac{\beta}{\varepsilon} y, \quad y^{\prime}=-\frac{\gamma}{\varepsilon} x+\frac{\alpha}{\varepsilon} y
$$

sera toujours de même nom que celle dont nous venons de parler. Il suffit pour s'en assurer, de remplacer dans l'expression (8) les entiers $\alpha, \beta, \gamma, \delta$ respectivement par $\frac{\delta}{\varepsilon},-\frac{\beta}{\varepsilon},-\frac{\gamma}{\varepsilon}, \frac{\alpha}{\varepsilon}$, ce qui changera cette expression en

$$
\frac{\alpha \delta-\beta \gamma}{\varepsilon^{2}}=\varepsilon .
$$

Les deux substitutions étant de même nature quant à la distinction que nous venons de faire, on peut transporter la dénomination précédente au groupe des deux formes et appeler l'équivalence de ces formes propre ou impropre suivant que la valeur de $\varepsilon$, commune aux deux substitutions en question, est +1 ou -1 . Il n'est pas nécessaire pour notre objet de considérer l'équivalence impropre qui au reste se change toujours en équivalence 
propre, si dans l'une des formes on change le signe du coëfficient moyeu. En disant donc désormais que deux formes sont équivalentes, nous entelldrons toujours qu'il s'agit de l'équivalence propre, ou autrement dit, qu'on peut passer de chacune de ces formes à l'autre, par une substitution telle que (2), où l'on a $\alpha \delta-\beta \gamma=1$. Pareillement, quand nous nous proposerons de découvrir toutes les transformations qui changent ces formes l'une dans l'autre, nous n'aurons en vue que celles qui satisfont à la condition précédente, et nous rejetterons toutes celles pour lesquelles on aurait $\alpha \delta-\beta \gamma=$ -1. Comme dans ce qui va suivre, il sera le plus souvent inutile de désigner les indéterminées par des lettres particulières, nous conviendrons d'indiquer une forme telle que (1), ou une substitution telle que (2), par ces notations abrégées

$$
(a, b, c), \quad\left(\begin{array}{l}
\alpha, \beta \\
\gamma, \delta
\end{array}\right)
$$

La notion de l'équivalence telle que nous venons de la fixer, donne lieu à ces théorèmes très-simples

I. Toute forme est équivalente à elle-même, puisqu'il est évident qu'elle ne varie pas, si on lui applique la subst. $\left(\begin{array}{c}1,0 \\ 0,1\end{array}\right)$.

II. Deux formes qui équivalent à une troisième, sont équivalentes entre elles. En effet, si la forme $f$, supposée équivalente à $f^{\prime}$, se transforme en celle-ci au moyen de la subst. $\left(\begin{array}{c}\alpha, \beta \\ \gamma, \delta\end{array}\right)$, et si celle-ci devient $\dot{a}$ son tour identique avec $f^{\prime \prime}$, au moyen de la subst. $\left(\begin{array}{c}\alpha^{\prime}, \beta^{\prime} \\ \gamma^{\prime}, \delta^{\prime}\end{array}\right)$, on passera évidemment de $f$ à $f^{\prime \prime}$, si l'on fait usage de la subst. unique $\left(\begin{array}{c}\alpha^{\prime \prime}, \beta^{\prime \prime} \\ \gamma^{\prime \prime},\end{array} \delta^{\prime \prime}\right)$, où l'on a

$$
\begin{array}{ll}
\alpha^{\prime \prime}=\alpha \alpha^{\prime}+\beta \gamma^{\prime}, & \beta^{\prime \prime}=\alpha \beta^{\prime}+\beta \delta^{\prime}, \\
\gamma^{\prime \prime}=\gamma \alpha^{\prime}+\delta \gamma^{\prime}, & \delta^{\prime \prime}=\gamma \beta^{\prime}+\delta \delta^{\prime},
\end{array}
$$

et il ne reste plus qu'à prouver qu'on a $\alpha^{\prime \prime} \delta^{\prime \prime}-\beta^{\prime \prime} \gamma^{\prime \prime}=1$. Mais cette équation résulte sur le champ de l'équation identique

$$
\alpha^{\prime \prime} \delta^{\prime \prime}-\beta^{\prime \prime} \gamma^{\prime \prime}=(\alpha \delta-\beta \gamma)\left(\alpha^{\prime} \delta^{\prime}-\beta^{\prime} \gamma^{\prime}\right) \text {, }
$$

où l'on a par hyp., $\alpha \delta-\beta \gamma=1$, et $\alpha^{\prime} \delta^{\prime}-\beta^{\prime} \gamma^{\prime}=1$.

La subst. $\left(\begin{array}{l}\alpha^{\prime \prime}, \beta^{\prime \prime} \\ \gamma^{\prime \prime},\end{array} \delta^{\prime \prime}\right)$, qui produit le même effet que les deux subst. $\left(\begin{array}{l}\alpha, \beta \\ \gamma, \beta\end{array}\right),\left(\begin{array}{l}\alpha^{\prime}, \beta^{\prime} \\ \gamma^{\prime},\end{array}, \delta^{\prime}\right)$, employées l'une après l'autre, peut s'appeler convenablement une substitution composée, où il importe de remarquer que l'ordre des substitutions composantes ne peut pas être interverti. 
III. Deux formes $(a, b, c)$ et $\left(a^{\prime}, b^{\prime}, c^{\prime}\right)$ étant supposées équivalentes, le plus grand diviseur commun des entiers $a, b, c$ est le mème que celui des entiers $a^{\prime}, b^{\prime}, c^{\prime}$, et la même égalité subsiste entre les deux groupes $a, 2 b, c$, et $a^{\prime}, 2 b^{\prime}, c^{\prime}$.

Comme l'équivalence admise suppose une transformation telle que (2), et par suite les équations (4), on voit immédiatement que tout diviseur commun de $a, b, c$, divise aussi $a^{\prime}, b^{\prime}, c^{\prime}$, et l'on arrive à un résultat semblable relativement aux groupes $a, 2 b$, $c$, et $a^{\prime}, 2 b^{\prime}, c^{\prime}$, si préalablement on suppose les deux membres de la seconde des équations (4), multipliés par '2. Un raisonnement analogue pouvant se faire en sens inverse, la proposition énoncée se trouve établie.

Nous observerons quil serait inutile de considérer des formes $(a, b, c)$ pour lesquelles le plus grand diviseur commun $\sigma$ de leurs coëfficients $a, b, c$ différerait de l'unité, puisque de pareilles formes ne sont évidemment que des formes du déterminant $\frac{D}{\sigma^{2}}$, affectées du facteur entier $\sigma$. Nous supposerons donc toujours $a, b$, $c$, libres de tout diviseur commun; cela ètant, le plus grand diviseur commun de $a, 2 b$, $c$, que nous désignerons constamment par $\omega$, ne peut avoir que l'une des trois valeurs $1,1+i$ ou 2 , ce qui donne lieu à diviser les formes quadratiques en trois espèces, appelées suivant l'ordre des cas énoncés, la première, la seconde ou la troisième, de sorte que des formes équivalentes sont toujours de même espèce.

\section{11.}

Relativement à l'équivalence des formes, il se présente deux questions principales à résoudre. Étant données deux formes ayant le même déterminant et appartenant $\dot{a}$ la même espèce, on peut demander $1^{\circ}$ si ces formes sont équivalentes ou non, et l'équivalence supposée reconnue, on peut se proposer $2^{\circ}$ d'assiguer toutes les substitutions par lesquelles ces formes se transforment l'une dans l'autre. Nous ne sommes pas pour le moment en mesure d'aborder la première de ces deux questions; mais nous pouvons traiter dès-à-présent la seconde, en la posant comme il suit:

"Elant données deux formes équivalentes ainsi qu'une transformation "de la première dans la seconde, trouver toutes les transformations "qui produisent le même effet."

I. I.a question énoncée peut se réduire à une autre plus simple et 
qni n'est au fond qu'une question particulière, mais de même nature que la proposée. Cette question particulière consiste à assigner toutes les substitutions par lesquelles une forme donnée se change en elle - même ou autrement dit, reste invariable quant $\dot{a}$ ses coëfficients. Pour le prouver, soit

$$
\text { (1) }\left(\begin{array}{ll}
\alpha, \beta \\
\gamma, \delta
\end{array}\right)
$$

la subst. donnée par laquelle la première $f$ des formes données se change dans la seconde $f^{\prime}$. Si maintenant l'on désigne par

$$
\text { (2) }\left(\begin{array}{c}
\lambda, \mu \\
\nu, \varrho
\end{array}\right)
$$

une subst. quelconque quii, étant appliquée à la forme $f$, reproduise cette même expression, il résulte du \$. précédent II., que par la subst. composée des précédentes, rangées dans l'ordre (2), (1),

où l'on a

$$
\text { (3) } \quad\left(\begin{array}{c}
\alpha^{\prime}, \beta^{\prime} \\
\gamma^{\prime}, \delta^{\prime}
\end{array}\right)
$$

$$
\text { (4) } \begin{cases}\alpha^{\prime}=\alpha \lambda+\gamma \mu, & \beta^{\prime}=\beta \lambda+\delta \mu, \\ \gamma^{\prime}=\alpha \nu+\gamma \rho, & \delta^{\prime}=\beta \nu+\delta \rho,\end{cases}
$$

$f$ se change en $f^{\prime}$. Cela posé, je dis que, si dans les équations (4.) on introduit successivement toutes les subst. (2), on obtiendra toutes les transformations possibles de $f$ en $f^{i}$, et de plus que chacune d'entre elles ne se présentera ainsi qu'une seule fois. Pour prouver d'abord ce dernier point, il suffit d'observer que les équations (4), en y considèrant $\lambda, \mu, \nu, \rho$ comme des inconnues, donnent ces valeurs complétement déterminées:

$$
\begin{aligned}
& \lambda=\delta \alpha^{\prime}-\gamma \beta^{\prime}, \quad \mu=\alpha \beta^{\prime}-\beta \alpha^{\prime}, \\
& \nu=\delta \gamma^{\prime}-\gamma \delta^{\prime}, \quad \rho=\alpha \delta^{\prime}-\beta \gamma^{\prime} .
\end{aligned}
$$

Reste à faire voir qu'il n'existe aucune transformation $\left(\begin{array}{c}\alpha^{\prime}, \beta^{\prime} \\ \gamma^{\prime}, \delta^{\prime}\end{array}\right)$ de $f$ en $f^{\prime}$, qui ue soit contenue dans les formules (4), en y considérant $\lambda, \mu, \nu, \rho$, généralement comme les coëfficients des subst. (2) défínies plus haut.

Comme la résolution des équations en question a douné des valeurs entières, et que d'un autre côté, on conclut de l'équation identique

combinée avec celles-ci

$$
(\lambda \rho-\mu \nu)(\alpha \delta-\beta \gamma)=\alpha^{\prime} \delta^{\prime}-\beta^{\prime} \gamma^{\prime},
$$

$$
\alpha \delta-\beta \gamma=1, \quad \alpha^{\prime} \delta^{2}-\beta^{\prime} \gamma^{\prime}=1,
$$

que l'on a aussi $\lambda \rho-\mu \nu=1$, tout revient évidemment à s'assurer que la subst. $\left(\begin{array}{l}\lambda, \mu \\ \nu, \varrho\end{array}\right)$ formée avec les entiers $\lambda, \mu, \nu, \rho$, donués par la résolution effectuée, 
est en effet l'une de celles qui changent la forme $f$ en elle-même. Désignant pour un instant par $\chi$, la forme encore inconnue dans laquelle $f$ se transforme par la subst. dont il s'agit, on voit d'abord que $\chi$ devient $f^{\prime}$ au moyen de la subst. $\left(\begin{array}{c}\alpha, \beta \\ \gamma, \delta\end{array}\right)$, et que par suite $f^{\prime}$ se change en $\chi$ au moyen de la subst. inverse $\left(\begin{array}{r}\delta,-\beta \\ -\gamma, \alpha\end{array}\right)$. Mais d'un autre côté, cette dernière change aussi $f^{\prime}$ en $f$, d'où il suit qu'on a $f=\chi$, ce qu'il s'agissait de pronver.

II. Tout se réduit donc à découvrir toutes les substitutions $\left(\begin{array}{ll}\lambda & \mu \\ \nu & \rho\end{array}\right)$ par lesquelles la forme donnée $f=(a, b, c)$, se change en elle-même. Pour résoudre cette question, il s'agira d'assigner toutes les valeurs entières $\lambda, \mu, \nu, \rho$ telles qu'en remplaçant dans l'expressiou

$$
a x^{2}+2 b x y+c y^{2}
$$

ou ce qui revient au mème, $a$ différant de zéro, dans celle-ci

$$
a\left(a x^{2}+2 b x y+c y^{2}\right) \text {, }
$$

$x$ et $y$ respectivement par $\lambda x+\mu y$ et $\nu x+\rho y$, cette expression reste identiquement la même. Nous ferons d'abord abstraction de la condition $\lambda \rho-\mu \nu=1$, toujours exigée dans les transformations que nous employons, et de plus nous considérerons $\lambda, \mu, \nu, \rho$ comme susceptibles de valeurs rationnelles quelconques. La question ainsi généralisée une fois résolue, il sera facile d'avoir égard aux conditions jusque-là négligées. L'expression dont il s'agit, se décompose en ces deux facteurs linéaires:

$$
(a x+(b+\vee D) y)(a x+(b-\checkmark D) y)
$$

où nous entendons par $\boldsymbol{V} \boldsymbol{D}$ une valeur déterminée, mais qui peut être arbitrairement choisie parmi les deux valeurs opposées généralement comportées par un radical quarré. La substitution indiquée change le produit précédent en celui-ci:

$[(a \lambda+b \nu+\nu \vee D) x+(a \mu+b \rho+\rho \vee D) y][(a \lambda+b \nu-v \checkmark D) x+(a \mu+b \rho-\rho \vee D) y]$. Désignant pour un instant les huit coëfficients par des lettres particulières, en posant $p=a, q=b+\checkmark D, \ldots, p^{\prime}=a \lambda+b \nu+\nu \vee D, \ldots$, l'égalité qu'il s'agit d'établir entre ces deux produits, s'écrira ainsi:

$$
(p x+q y)(\boldsymbol{r} x+s y)=\left(p^{\prime} x+q^{\prime} y\right)\left(\boldsymbol{r}^{\prime} x+\boldsymbol{s}^{\prime} y\right) \text {. }
$$

Or, les quatre constantes $p, q, r, s$ données étant évidemment toutes différentes de zéro, les conditions nécessaires et suffisantes pour l'identité de ces deux expressions, exigent évidemment qu'on ait $1^{\circ} \frac{p^{\prime} r^{\prime}}{p r}=1$, et en 
outre $2^{\circ}$ l'un ou l'autre de ces deux systèmes d'équations

$$
\frac{p^{\prime}}{p}=\frac{q^{\prime}}{q}, \quad \frac{r^{\prime}}{r}=\frac{s^{\prime}}{s} ; \quad \frac{r^{\prime}}{p}=\frac{s^{\prime}}{q}, \quad \frac{p^{\prime}}{r}=\frac{q^{\prime}}{s} .
$$

Si maintenant, suivant qu'il s'agit du premier ou du second cas, on pose

$$
\frac{p^{\prime}}{p}=\varphi+\psi r D, \quad \text { ou } \quad \frac{r^{\prime}}{p}=\varphi+\psi r D,
$$

où nous supposons $\phi$ et $\psi$ rationnels, ce qui est permis, les coëfficients $p, q, \ldots$ ne renfermant que la seule irrationnelle $\checkmark D$, on aura respectivement

$$
\frac{r^{\prime}}{r}=\varphi-\psi \vee D, \quad \text { ou } \quad \frac{p^{\prime}}{r}=\varphi-\psi \vee D D,
$$

et l'équation $\frac{p^{\prime} r^{\prime}}{p r}=1$, commune aux deux cas, prendra la forme

$$
\text { (5) } \varphi^{2}-D \psi^{2}=1 \text {. }
$$

Quant ̇̇ l'autre condition exprimée par deux équations, il suffit d'écrire pour l'un et l'autre cas, la première de ces deux équations, celle-ci comprenant virtuellement la seconde qui n'en diffère que par le signe du radical. On aura donc suivant les deux cas

ou

$$
\frac{a \lambda+b \nu+v \sqrt{D}}{a}=\frac{a \mu+b \varrho+\varrho \sqrt{D}}{b+\sqrt{D}}=\varphi+\psi \vee D,
$$

$$
\frac{a \lambda+b \nu-\nu \sqrt{D}}{a}=\frac{a \mu+b \varrho-\rho \sqrt{D}}{b+\sqrt{D}}=\varphi+\psi \vee D .
$$

En égalant séparément dans ces formules les parties rationnelles et les coëfficients de $\boldsymbol{V} \boldsymbol{D}$, et en résolvant ensuite les équations que l'on obtient ainsi, par rapport $\grave{a} \lambda, \mu, \nu, \rho$, on trouve sans indétermination et suivant les deux cas:

$$
\begin{array}{l|l}
\lambda=\varphi-b \psi, & \lambda=\varphi+b \psi, \\
\mu=-c \psi, & \mu=\frac{2 b}{a} \varphi+\frac{b^{2}+D}{a} \psi, \\
\nu=a \psi, & \nu=-a \psi, \\
\rho=\varphi+b \psi, & \rho=-\phi-b \psi .
\end{array}
$$

On voit donc que toutes les valeurs rationnelles $\lambda, \mu, \nu, \rho$ qui satisfont $\dot{a}$ la condition d'invariabilité exigée, sont données par ces deux systèmes de formules très-simples, où $\varphi$ et $\psi$ désignent généralement toutes les valeurs rationnelles simultanées compatibles avec l'équation (5). Il s'agit maintenant d'avoir égard aux conditions que nous avons négligées, et dont l'une est exprimée par l'équation $\lambda \rho-\mu \nu=1$. La substitution des expressions 
précédentes moutre par un calcul très-simple, que le premier système y satisfait, tandis que relativement au second, on trouve $\lambda_{\rho}-\mu \nu=-1$. Ce dernier devant aiusi être rejeté, il ne reste plus qu'à examiner sous quelles conditions les expressions de $\lambda, \mu, \nu, \rho$, données par le premier système, sont entières. Il est facile de voir que cela exige que les produits $\omega \phi, \omega \psi, \omega$ désignant toujours le plus grand diviseur commun de $a$, $2 b, c$, soient des entiers. En effet, comme des équations précédentes on conclut facilement

$$
\nu=\frac{a}{\omega} \omega \psi, \quad \rho-\lambda=\frac{2 b}{\omega} \omega \psi, \quad-\mu=\frac{c}{\omega} \omega \psi,
$$

on voit que, si le produit $\omega \psi$, réduit à sa plus simple expression, avait un dénominateur autre que l'unité, ce dénominateur serait diviseur commun des entiers $\frac{a}{\omega}, \frac{2 b}{\omega}, \frac{c}{\omega}$, qui n'admettent pas de pareil diviseur. La conclusion obtenue pour $\omega \psi$, s'étend sur le champ à $\omega \varphi$, au moyen de l'équation $\omega \emptyset$ $=\omega \lambda+b \omega \psi$. Mais la réciproque a également lieı, et il est facile de s'assurer que, si l'on fait usage de valeurs de $\phi$ et $\psi$, telles que $\phi=\frac{t}{\omega}, \psi=\frac{u}{\omega}$, où $t$ et $u$ sont des entiers, et satisfaisant à l'équation ( $\tilde{b})$, il en résultera des vạleurs entières pour $\lambda, \mu, \nu, \rho$. Pour le voir, substituons ces expressions dans les équations obtenues plus haut; il vieudra ainsi

(6) $t^{2}-D u^{2}=\omega^{2}$

(7) $\lambda=\frac{t-b u}{\omega}, \quad \mu=-\frac{c u}{\omega}, \quad y=\frac{a u}{\omega}, \quad \rho=\frac{t+b u}{\omega}$.

Relativement $\dot{a} \mu$ et $\nu$ il n'y a rien à prouver, $a$ et $c$ élant divisibles par $\omega$. Quant ̀̀ $\lambda$ et $\rho$, comme leur différence $\rho-\lambda=\frac{2 b u}{\omega}$, est évidemment un entier, tout revieut à faire voir que l'une des expressions $\frac{t+b u}{\omega}, \frac{t-b u}{\omega}$, est pareillement un entier. Mais de l'équation à laquelle $t$ et $u$ sont supposés satisfaire, mise sous la forme $\frac{(t+b u)(t-b u)}{\omega^{2}}=1-\frac{a c}{\omega^{2}} u^{2}$, on conclut que le produit des deux facteurs $t+b u$ et $t-b u$ est un multiple de $\omega^{2}$, d'où et de ce que $\omega$ ne renferme pas plusieurs nombres premiers différents, il suit que l'un au moins des deux facteurs est divisible par $\omega$, ce qu'il s'agissait de faire voir. Les formules ( 7 ), en y substituant successivement toutes les solutions entières de l'équation (6), donneront donc toutes les 
transformations $\left(\begin{array}{c}\lambda, \mu \\ \varrho, v\end{array}\right)$ de la forme $(a, b, c)$ en elle-mène, et il est d'ailleurs évident que chacune de ces transformations ne se présentera qu'une seule fois, car on voit par les deux premières des formules en question, qu'à des valeurs déterminées $\lambda$ et $\mu$ répondent toujours des valeurs également déterminées pour $t$ et $u$.

Remarque. L'analyse qui vient de nous conduire de la manière la plus simple à la solution de la question proposée, a en outre l'avantage de montrer clairement ce qui distingue les transformations propres, les seules que nous ayons à considérer, de celles qu'on appelle impropres. On voit en effet que, s'il s'agit des transformations d'une forme en elle-même, les premières sont celles pour lesquelles les deux expressions linéaires dont la forme donnée peut être considérée comme le produit, restent l'une et l'autre invariables, abstraction faite des facteurs constants qu'elles acquièrent; tandis que les transformations impropres qui n'existent toutefois que pour des formes d'une nature particulière et répondent alors au second des deux systèmes d'équations obtenus plus haut, ont pour effet d'échanger entre elles les deux expressions linéaires dont il s'agit. La même remarque s'étend aux substitutions qui ne reproduisent pas la forme donnée, et la changent au contraire en une autre équivalente mais distincte. En combinant ce qui précède avec le résultat lu ${ }^{\circ}$ précédent, il est facile de s'assurer que, si après avoir décomposé en facteurs linéaires la forme primitive et celle qui en dérive, on considère comme correspondants ceux de leurs facteurs, qui contiennent le radical $\boldsymbol{V} D$ avec le même signe, toute transformation de la première dans la seconde, sera propre ou impropre, suivant que les facteurs linéaires se changent en leurs correspondants ou non.

III. Si maintenant nous substituons les expressions ( 7 ) dans les équations (4), ces dernières prendront la forme:

$$
\begin{array}{ll}
\alpha^{\prime}=\frac{a t-(b a+c \gamma) u}{\omega}, & \beta^{\prime}=\frac{\beta t-(b \beta+c \delta) u}{\omega}, \\
\gamma^{\prime}=\frac{\gamma t+(a \alpha+b \gamma) u}{\omega}, & \delta^{\prime}=\frac{\delta t+(a \beta+b \delta) u}{\omega} .
\end{array}
$$

Au moyen de ces équations, on pourra donc, une première transformation $\left(\begin{array}{c}\alpha, \beta \\ \gamma, \delta\end{array}\right)$ d'uue forme $(a, b, c)$ en une autre $\left(a^{\prime}, b^{\prime}, c^{\prime}\right)$ équivalente étant donnée, en déduire toutes les transformations possibles, en supposant d'ailleurs que la solution complète de l'équation (6) soit également connue. 
\$. 12.

Lorsque la forme

$$
\text { (1) } a x^{2}+2 b x y+c y^{2}
$$

dont nous désignerons le déterminant par $D$, et dont nous considérerons d'abord les coëfficients $a, b, c$, comme susceptibles d'un diviseur commun quelconque, obtient une valeur déterminée $m$, en y attribuant des valeurs particulières $r$ et $s$ aux indéterminées $x$ et $y$, nous dirons que l'entier $m$ est représenté par la forme donnée. Nous supposerons toujours, si nous n'avertissons expressément du contraire, que les entiers déterminés $r$ et $s$ sont premiers entre eux. Sous cette restriction, $m$ diffère toujours de zéro, car il est facile de voir que l'hypothèse de $m=0$, suppose $r=0, s=0$, valeurs dont un nombre quelconque est diviseur commun. Il s'agit maintenant de déduire les conséquences qui résultent d'une représentation telle que nous venons de la définir. On voit tout d'abord que, si l'on choisit deux entiers $\rho$ et $\sigma$ qui satisfassent à l'équation

$$
\text { (2) } r \sigma-s \rho=1 \text {, }
$$

évidemment résoluble, et que l'on applique ensuite la substitution $\left(\begin{array}{c}r, \rho \\ s, \\ \sigma\end{array}\right)$ a la forme (1), celle-ci se changera en cette autre équivalente

$$
\text { (3) }\left(m, n, \frac{n^{2}-\boldsymbol{D}}{m}\right) \text {, }
$$

où
(4) $m=a r^{2}+2 b r s+c s^{2}$
(5) $n=a r \rho+b(r \sigma+s \rho)+c s \sigma$.

Le troisième coëfficient de la forme (3) étant entier, on conclut que $n$ satisfait à la congruence

$$
\text { (6) } z^{2} \equiv D(\bmod . m) \text {. }
$$

On voit donc qu'une condition nécessaire, quoique nullement suffisante, pour que $m$ puisse être représenté par la forme (1), consiste en ce que $\boldsymbol{D}$ doit être résidu quadratique relativement au module $m$, et que d'une représentation supposée connue, on peut toujours déduire une racine $n$ de la congruence (6), en substituant une solution quelconque de l'équation (2) dans la formule (5). Comme l'équation (2) admet toujours une infinité de solutions, il est naturel de rechercher comment $n$ varie, lorsqu'on passe d'une de ces solutions à une autre. Pour y parvenir, soit $\rho_{0}, \sigma_{0}$ une solution particulière et soit $n_{0}$ la valeur correspondante de $n_{\text {; }}$ si maintenant l'ou introduit la solution générale $\rho=\rho_{0}+r \xi, \sigma=\sigma_{0}+s \xi$, où $\xi$ désigne un 
entier complexe arbitraire, dans la formule (5), on aura pour la valeur générale de $n$,

$$
n=n_{0}+m \xi
$$

d'où l'on conclut que les valeurs en nombre infini, dont $n$ est susceptible, étant toutes congrues entre elles suivant le module $m$, forment une racine unique de la congruence (6), et l'on doit ajouter que l'arbitraire $\xi$ peut toujours être choisie de manière à faire coïncider $n$ avec l'une quelconque des valeurs en nombre infini, que l'on peut considérer comme autant d'expressions différentes d'une même racine de la congruence en question.

Cela étant, nous dirons désormais d'une manière abrégée, que la représentation de l'entier $m$ par la forme (1), pour laquelle on a $x=r$, $y=s$, appartient à la valeur $n$ de l'expression $\checkmark D(\bmod . m)$, que l'on déduit de l'équation (5), en y substituant deux quelconques des entiers $\rho$ et $\sigma$ qui satisfont à l'équation (2).

La conclusion que nous venons d'obtenir et qui consiste en ce que la représentation $x=r, y=s$, appartenant à la valeur $n$ de $\boldsymbol{V D}(\bmod . m)$, a toujours pour conséquence l'équivalence des formes (1) et (3), a également lieu en sens inverse. En effet si, supposant l'équivalence de ces dernières, nous désignons par $\left(\begin{array}{c}r, \rho \\ s, \\ \sigma\end{array}\right)$ l'une quelconque des substitutions par lesquelles la première se change dans la seconde, nous aurons évidemment les équations (2), (4) et (5), dont la seconde fournit une représentation qui en vertu des deux autres appartient évidemment à la valeur $n$ de $\checkmark \boldsymbol{D}$ (mod. $m$ ). Je dis de plus qu'il n'y aucune représentation satisfaisant à la condition exigée, qui ne puisse s'obtenir ainsi au moyen d'une transformation de la forme (1) en celle (3), et que chaque représentation se présentera une seule fois, c'est-à-dire qu'elle proviendra toujours d'une transformation unique et déterminée. Pour prouver d'abord le premier point, remarquons qu'en vertu de la définition même de la valeur $n$ à laquelle une représentation est dite appartenir, supposer l'existence d'une telle représentation pour l'entier $m$, c'est supposer les équations (4), (2) et (5), desquelles il résulte sur le champ que la forme (1), au moyen de la substitution $\left(\begin{array}{l}r, e \\ s, \sigma\end{array}\right)$, se change en une autre du même déterminant $D$, et dont les deux premiers coëfficients sont $m$ et $n$. On conclut de là que le troisième coëfficient est $\frac{n^{2}-D}{m}$, et que la substitution indiquée est en effet l'une de celles par les- 
quelles la forme (1) se change en celle (3). Quant au second point, il est évident que pour l'établir, on n'a qu'à faire voir que les deux équations (2) et (5), en y considérant $r$ et $s$ comme donnés, ne sauraient être satisfaites par plus d'un couple de valeurs de $\rho$ et de $\sigma$. Mais cela est manifeste, puisque les équations dont il s'agit, étant résolues, donnent ces valeurs complètement déterminées

$$
\rho=\frac{(n-b) r-c s}{m}, \quad \sigma=\frac{a r+(n+b) s}{m} .
$$

On voit par ce qui précède, que pour que l'entier $m$ puisse être représenté par la forme (1) de manière à ce que ces représentations appartiennent à une valeur donnée $n$ de l'expression $\checkmark D(\bmod m)$, il faut et il suffit que les formes (1) et (3) soient équivalentes entre elles. Cette condition supposée remplie, on n'aura plus qu'à chercher toutes les substitutions $\left(\begin{array}{l}r, \varrho \\ s, \\ \sigma\end{array}\right)$ par lesquelles la forme (1) se change en celle (3), et l'on posera $x=r$, $y=s$. Or, les substitutions dont il s'agit ayant été exprimées dans le $\$$. précédent en fonction de l'une quelconque d'entre elles, on en conclut, si nous revenons maintenant à l'hypothèse que les coëfficients de la forme (1) n'out pas de diviseur commun, que les représentations cherchées sont toutes comprises dans ces deux équations

$$
x=\frac{\alpha t-(b a+c \gamma) u}{\omega}, \quad y=\frac{\gamma t+(a \alpha+b \gamma) u}{\omega},
$$

où $\alpha, \gamma$ sont resp. le premier et le troisième des coëfficients qui entrent dans une substitution $\left(\begin{array}{c}\alpha, \beta \\ \gamma, \delta\end{array}\right)$ arbitrairement choisie parmi celles qui transforment la forme (1) en celle ( 3 ), et où $t$ et $u$ satisfont généralement à l'équation $t^{2}-D u^{2}=\omega^{2}$. Il est bon de remarquer que le résultat est maintenant tout-à-fait indépendant de la forme (3) que nous avons eu à considérer pour l'obtenir. En effet, comme $x=\alpha, y=\gamma$ est évidemment une représentation particulière comprise dans les formules précédentes et qui s'en déduit en supposant $t=\omega, u=0$, on peut l'énoncer en disant que les équations que nous venons d'obtenir, expriment toutes les représentations appartenant à une même valeur de $\boldsymbol{V} \boldsymbol{D}(\bmod . m)$, en fonction de l'une quelconque d'entre elles. 


\section{\$. 13.}

Les questions que nous avons traitées daus les \$5. précédents, s'étant trouvées dépendre de la solution de l'équation indéterminée

$$
t^{2}-D u^{2}=\omega^{2}
$$

il est temps de nous occuper de cette dernière. Mais pour ne pas domer une étendue démesurée au présent Mémoire, nous considérerons exclusivement le cas où $\omega=1$, cas qui est celui des formes de première espèce; et nous laisserons au lecteur qui voudrait s'exercer sur ces matières, le soin de chercher les modifications assez légères qu'il faudrait apporter aux recherches suivantes pour les rendre applicables aux formes des deux autres espèces.

La théorie de l'équation

$$
t^{2}-D u^{2}=1
$$

peut se déduire d'un lemme dont voici l'énoncé :

„a désignant un nombre complexe irrationnel donné, on pourra tou„,jours trouver une infinité d'entiers complexes simultanés $x$ et $y$, tels "qu'on ait

$$
\mathbf{N}(\boldsymbol{x}-\boldsymbol{a y})<\frac{4}{\mathbf{N}(y)}
$$

Observons d'abord que, si l'on satisfait à la condition du lemme par le sy-. stème $x, y$, on y satisfera aussi par celui-ci $i^{\circ} x$, $i^{\circ} y$. Comme dans l'application que nous aurons à faire du lemme, il importe de ne pas employer simultanément des systèmes dérivant ainsi l'un de l'autre, nous éviterons cet inconvenient, en ne considérant deux systèmes comme distincts qu'autant que les valeurs de $\boldsymbol{N}(\boldsymbol{x}-\boldsymbol{a y})$ qui s'y rapportent, sont différentes entre elles. Il est en effet évident que pour deux systèmes comme ceux dout il vient d'être question, l'expression $N(x-a y)$ a toujours la même valeur. On voit encore que la condition du lemme se trouve remplie, lorsque, $x$ étant quelconque, on a $y=0$, mais nous ferons pareillement abstraction de ce cas, de sorte que $x$-ay aura toujours une valeur irrationnelle et par conséquent différente de zéro.

Pour démontrer notre lemme, commençons par faire voir qu'on peut toujours trouver deux entiers $x$ et $y$, qui satisfaisant à l'inégalité proposée, soient en outre tels que l'on ait

$$
\boldsymbol{N}(\boldsymbol{x}-\boldsymbol{a y})<\boldsymbol{A},
$$

$A$ désiguant une quantité positive arbitrairement choisie. Soit à cet effet 
$n$ un entier positif pour lequel on ait, $A>\frac{1}{2 n^{2}}$, et désignons par $\eta$ l'un quelconque des entiers complexes dont les deux parties, et j'entends par là la partie réelle et le coëfficient de $i$ qui entrent dans une expression complexe quelconque, soient comprises dans la suite

$$
-n,-(n-1), \ldots,-1,0,+1, \ldots, n-1, n \text {. }
$$

Relativement à chacun des entiers $\eta$ dont le nombre est évidemment égal a $(2 n+1)^{2}$, déterminons l'entier correspondant $\xi$ tel que les deux parties de l'expression

$$
\text { - } \xi-a \eta,
$$

obtiennent des valeurs non-négatives et inférieures à l'unité. Cela supposé, il est évident que, si l'on désigne par

$$
p \frac{1}{2 n}, \quad q \frac{1}{2 n},
$$

les plus grand multiples de $\frac{1}{2 n}$, resp. contenus dans les deux parties dont il s'agit, les entiers réels $p$ et $q$ seront l'un et l'autre compris dans la suite

$$
0,1,2, \ldots, 2 n-1 \text {. }
$$

Or, comme avec de pareils entiers on ne peut former qu'un nombre de combinaisons distinctes, exprimé par $(2 n)^{2}$, tandis que celui des expressions $\xi-a_{\eta}$ est $(2 n+1)^{2}$, on voit que l'une au moins des combinaisons $p, q$ devra se reproduire. Soient donc

$$
\xi-a \eta, \quad \xi^{\prime}-a \eta^{\prime},
$$

les deux expressions ou deux des expressions pour lesquelles cette circonstance se présente, il est évident qu'en formant la différence de ces expressions, on obtiendra, en posant

$$
\xi-\xi^{\prime}=x, \quad \eta-\eta^{\prime}=y
$$

une nouvelle expression

$$
x-a y,
$$

dans laquelle l'entier $y$ sera évidemment différent de zéro, et dont les deux parties seront, abstraction faite du signe, inférieures $\grave{a} \frac{1}{2 n}$, de sorte qu'on aura

$$
N(x-a y)<\frac{1}{2 n^{2}} \text {, et par suite } N(x-a y)<A,
$$

ce qui coïncide avec la seconde des conditions posées plus haut. Pour prouver que l'expression $N(x-a y)$ satisfait aussi à l'autre condition qui 
est celle du lemme, observons que, les deux parties de $y=\eta-\eta^{\prime}$, ayant évidemment des valeurs numériques non-supérieures à $2 n$, on a l'inégalité $\boldsymbol{N}(\boldsymbol{y}) \leq 8 \boldsymbol{n}^{2}$, dont la comparaison avec celle que nous venons d'obtenir, doune

conformément à l'énoncé.

$$
N(x-a y)<\frac{4}{N\left(y^{\prime}\right)}
$$

Ayant ainsi prouvé qu'on peut toujours trouver un couple $x, y$, qui, en même temps qu'il s'accorde avec la condition de l'énoncé, satisfasse à l'inégalité $\boldsymbol{N}(x-a y)<\boldsymbol{A}$, où $\boldsymbol{A}$ est d'une petitesse arbitraire, il est facile d'en conclure la vérité du lemme. Il suffit pour cela d'observer que, quel que soit le nombre des systèmes qu'on suppose déjà connus, on trouvera un nouveau système distinct des premiers, si, appliquant le procédé que nous venons d'exposer, on y suppose $\boldsymbol{A}$ égal à la plus petite des valeurs que l'expression $\mathbf{N}(x-a y)$ présente dans les systèmes antérieurement obtenus.

Remarquons maintenant que relativement à deux quantités complexes quelconques $r$ et $s$, on a l'inégalité connue et d'ailleurs facile a vérifier,

$$
\checkmark \boldsymbol{N}(\boldsymbol{r}+\boldsymbol{s}) \leqq \boldsymbol{N}(\boldsymbol{r})+\boldsymbol{V} \boldsymbol{N}(\boldsymbol{s})
$$

les radicaux étant supposés pris positivement. Supposant $r=x-a y$, $s=2 a y$, il viendra

$$
\checkmark N(x+a y) \leqq \checkmark N(x-a y)+\checkmark N(2 a y)
$$

inégalité qui au moyen de celle du lemme, mise sous la forme

se change en

$$
\checkmark N(x-a y)<\frac{2}{\sqrt{N}(y)},
$$

$$
\checkmark N(x+a y)<2 \checkmark N(a y)+\frac{2}{\sqrt{N}(y)} .
$$

Ces deux dernières étant multipliées entre elles, donnent

$$
\checkmark \boldsymbol{N}\left(x^{2}-a^{2} y^{2}\right)<4 \checkmark N(a)+\frac{4}{N(y)}
$$

et par suite, $y$ étant un entier complexe différent de zéro de sorte que $\boldsymbol{N}(\boldsymbol{y}) \geq \mathbf{1}$

$$
\checkmark N\left(x^{2}-a^{2} y^{2}\right)<4(\checkmark N(a)+1) \text {. }
$$

On voit donc que pour tous les couples d'entiers qui satisfont au lemme et dont le nombre est infini, $N\left(x^{2}-a^{2} y^{2}\right)$ reste au dessous d'une limite invariable. Appliquons ce résultat au cas où $a=\checkmark D, D$ élant un entier complexe non-quarré et le radical désignant une racine déterminée qui restera toujours la même dans ce qui va suivre. Comme dans cette hypo- 
thèse, $x^{2}-a^{2} y^{2}=x^{2}-D y^{2}$ est un entier complexe et qu'il n'y a qu'un nombre fini d'entiers dont la norme soit imférieure à une limite donnée, il faudra nécessairement que l'expression $x^{2}-D y^{2}$ obtienne une infinité de fois une même valeur $l$ qui sera évidemment différente de zéro, $y$ n'étant pas nul. L'équation $x^{2}-D y^{2}=l$, étant ainsi satisfaite par un nombre infini de systèmes $x, y$, on voit encore que parmi ces systèmes il s'en trouvera nécessairement un nombre illimité, pour lesquels les valeurs tant de $x$ que de $y$ présentent des différences multiples de $l$. Soient

$$
x^{2}-D y^{2}=l, \quad x^{\prime 2}-D y^{\prime 2}=l,
$$

deux équations pour lesquelles cela arrive, de sorte qu'on ait simultanément $x \equiv x^{\prime}, y \equiv y^{\prime}(\bmod . l)$. Le produit de ces équations étant

$$
\left(\boldsymbol{x} x^{\prime}-D y y^{\prime}\right)^{2}-D\left(x y^{\prime}-y x^{\prime}\right)^{2}=l^{2},
$$

et $x y^{\prime}-y x^{\prime}$ étant divisible par $l$ en vertu des conditions supposées, $\boldsymbol{x} x^{\prime}-D y y^{\prime}$ sera aussi un multiple de $l$, de sorte qu'en divisant par $l^{2}$, on aura

$$
t^{2}-D u^{2}=1
$$

les entiers $t$ et $u$ étant donnés par les formules

$$
t=\frac{x x^{\prime}-\boldsymbol{D} y \underline{y}^{\prime}}{l}, \quad u=\frac{x y^{\prime}-y}{l} \underline{x}^{\prime} .
$$

Nous ajouterons qu'il ne saurait arriver qu'on eût $u=0$, car il est facile de se convaincre que cela supposerait $x^{\prime}= \pm x, y^{\prime}= \pm y$, de sorte que les systèmes $x, y$ et $x^{\prime}, y^{\prime}$ ne seraient pas distincts.

Etant ainsi assuré que l'équation $t^{2}-D u^{2}=1$, est toujours résoluble sans qu'on suppose $u=0$, on parviendra nécessairement $\dot{a}$ une solution si l'on attribue successivement $\dot{a} \boldsymbol{u}$ toutes les valeurs entières dont les normes forment la suite croissante des entiers positifs susceptibles d'être décomposés en deux quarrés, jusqu'à ce que l'on tombe sur une valeur de $u$ pour laquelle $D u^{2}+1$ soit égal à un quarré. Cette simple possibilité suffit pour notre objet. Il existe un algorithme assez expéditif et analogue à celui des fractions connues, au moyen duquel on peut obtenir toutes les solutions de l'équation proposée ou plutòt celle de ces solutions, que l'on doit considérer comıne fondamentale et dont les autres se déduisent facilement; mais comme l'exposition de cet algorithme exigerait de longs détails qui ne sont nullement nécessaires pour le but que nous avons en vue, nous ne nous en occuperons pas ici. 


\section{\$. 14.}

La possibilité de l'équation

$$
\text { (1) } t^{2}-D u^{2}=1
$$

ayant été établie dans le \$. précédent, il s'agira maintenant de découvrir le lien qui existe entre ces solutions en nombre infini. C'est à quoi nous parviendrons par les considérations suivantes.

I. Observons d'abord que la double solution évidente $t= \pm 1, u=0$, est la seule pour laquelle l'une des indéterminées soit égale à zéro. Car il est manifeste que l'hypothèse $t=0$, est inadmissible, $D$ n'élant pas un quarré. Pour cette solution on a $N(t+u v D)=1$, et je dis de plus qu'elle est la seule pour laquelle cette équation ait lieu. En effet, comme les expressions $N(t+u \vee D), N(t-u \vee D)$ ont toujours des valeurs réciproques l'une de l'autre, puisque l'on a $N(t+u \vee D) N(t-u \vee D)=$ $\boldsymbol{N}\left(t^{2}-D u^{2}\right)=1$, la condition précédente est équivalente à celle-ci

$$
\boldsymbol{N}(t+u \boldsymbol{V} \boldsymbol{D})+\mathbf{N}(t-u \boldsymbol{v} \boldsymbol{D})=2 .
$$

Si maintenant l'on remarque que, $r$ et $s$ étant des quantités complexes quelconques, on a identiquement

$$
\boldsymbol{N}(\boldsymbol{r}+s)+\boldsymbol{N}(\boldsymbol{r}-\boldsymbol{s})=2 \boldsymbol{N}(\boldsymbol{r})+2 \mathbf{N}(s)
$$

cette dernière pourra prendre la forme

$$
\boldsymbol{N}(t)+\boldsymbol{N}(\boldsymbol{u}) \mathbf{N}(\boldsymbol{V} \boldsymbol{D})=1 .
$$

Or, $N(\checkmark D)$ étant une quantité égale ou supérieure à l'unité, cette équation exige évidemment que l'on ait $u=0$, ou $t=0$, lorsque $\boldsymbol{N}(\boldsymbol{D})=1$; mais la dernière hypothèse ne pouvant avoir lieu, l'assertion avancée se trouve justifiée.

II. Je dis en second lieu que, si pour deux solutions $t$, $u$, et $t^{\prime}, u^{\prime}$, on a $N\left(t^{\prime}+u^{\prime} \vee D\right)=N(t+u \vee D)$, ces deux solutions sont ou identiques ou opposées, de sorte que $t^{\prime}= \pm t, u^{\prime}= \pm u$, les signes se correspondant. En effet il est clair que de deux solutions quelconques $t$, $u$, et $t^{\prime}, u^{\prime}$, on peut en déduire une troisième au moyen de l'équation

$$
\frac{t^{\prime}+u^{\prime} \sqrt{D}}{t+u \sqrt{D}}=\tau+u \vee D
$$

dans laquelle il faut égaler séparément les parties rationnelles et les coëfficients de $\boldsymbol{r} \boldsymbol{D}$, ce qui donne

$$
\tau=t t^{\prime}-D u u^{\prime}, \quad v=t u^{\prime}-u t^{\prime}
$$


Comme relativement $\dot{a}$ cette nouvelle solution on a

$$
\boldsymbol{N}(\tau+u \boldsymbol{v D})=\frac{\boldsymbol{N}\left(\boldsymbol{t}^{\prime}+u^{\prime} \sqrt{D}\right)}{\boldsymbol{N}(\boldsymbol{t}+u \sqrt{\boldsymbol{D})}}=1,
$$

et par suite $\tau= \pm 1, v=0$, on conclut $t^{\prime}= \pm t, u^{\prime}= \pm u$, ce qu'il s'agissait de prouver.

III. Si l'ou excepte la double solution $t= \pm 1, u=0$, les solutions de l'équation (1) existent toujours par groupes de quatre, les indéterminées pouvant être prises avec un signe arbitraire. Il est évident que relativement $\dot{a}$ un pareil groupe, l'expression $t+u \sqrt{ } D$ a quatre valeurs distinctes exprimées par $\pm \chi, \pm \frac{1}{\chi}, \chi$ désignant l'une quelconque d'entre elles, tandis que l'expression $N(t+u \sqrt{ } D)$ ne présente que ces deux valeurs distinctes $\boldsymbol{N}(\chi), \boldsymbol{N}\left(\frac{1}{\chi}\right)$, réciproques l'une de l'autre. L'expression $\mathbf{N}(t+u \checkmark D)$ n'ayant qu'une valeur unique supérieure à l'unité pour chaque groupe, cette valeur pourra servir à caractériser ce groupe et à le distinguer de tous les autres, comme cela résulte $\mathrm{du} \mathrm{n}^{\circ}$ précédent où l'on a vu que la supposition $N(t+u \vee D)=N\left(t^{\prime}+u^{\prime} \vee D\right)$, ne peut avoir lieu

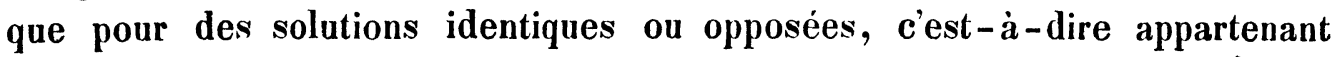
au même groupe. Cela posé, nous appellerons groupe fondamental ' celui pour lequel la valeur de $\boldsymbol{N}(t+u \mathfrak{V D})$, toujours supposée supérieure à l'unité, est moindre que la valeur analogue relative à tout autre groupe. Si maintenant l'on remarque que, la variable positive $\varrho$ étant supposée croître $\dot{a}$ partir de $\varrho=1$, la fonction $\varrho+\frac{1}{\varrho}$ croîtra également $\dot{a}$ partir de la valeur 2 , on voit que la définition précédente revient $\dot{a}$ dire que le groupe fondamental est celui pour lequel l'expression

$$
\begin{aligned}
\boldsymbol{N}(t+u \vee D)+\frac{1}{N(t+u \sqrt{ } \boldsymbol{D})} & =\boldsymbol{N}(\boldsymbol{t}+\boldsymbol{u} \vee \boldsymbol{D})+\boldsymbol{N}(\boldsymbol{t}-\boldsymbol{u} \vee \boldsymbol{D}) \\
& =2 \boldsymbol{N}(\boldsymbol{t})+2 \boldsymbol{N}(\boldsymbol{u}) \boldsymbol{N}(\boldsymbol{V D})
\end{aligned}
$$

a la plus petite valeur supérieure à 2 . Sous cette forme, la définition, quoique la même au fond, a l'avantage d'être indépendante de la supposition $\boldsymbol{N}(t+u \sqrt{ } \boldsymbol{D})>1$, l'expression précédente ayant évidemment la même valeur pour chacune des quatre solutions formant un même groupe. II est actuellement facile d'indiquer une méthode propre à faire découvrir le groupe fondamental, en supposant toujours qu'il s'agisse de simples possibilités et nullement d'une opération facile sous le rapport du calcul pratique. Ayant 
trouvé une première solution $t, u$, et déterminé la valeur correspondante de $\boldsymbol{N}(t+u \boldsymbol{V} \boldsymbol{D})$, désignée par $b$, tout revient à voir quels sont parmi les couples d'entiers $t$ et $u$, tels qu'on ait

$$
1<\mathbf{N}(t)+\mathbf{N}(u) \mathbf{N}(\boldsymbol{V} \boldsymbol{D}) \leqq \frac{1}{2}\left(b+\frac{1}{b}\right),
$$

et qui sont évidemment en nombre fini, ceux qui satisfaisant à l'équation (1), donnent la plus petite valeur à l'expression qui vient d'être écrite. Les quatre couples qui remplissent ces conditions, coïncident avec les quatre solutions du groupe cherché.

Il nous reste à faire voir comment de l'une des solutions de ce groupe l'on peut déduire toutes les solutions de la proposée. Quoique cela puisse se faire au moyen de l'une quelconque d'entre elles, nous conviendrons, pour éviter des distinctions tout-à-fait inutiles, de nous servir constamment de l'une des deux solutions opposées pour lesquelles on a $\boldsymbol{N}(t+u \vee D)>1$. Nous désignerons par $\boldsymbol{T}, \boldsymbol{U}$, celle des solutions fondamentales que nous emploierous et nous poserons $\boldsymbol{N}(\boldsymbol{T}+\boldsymbol{U} \boldsymbol{V} \boldsymbol{D})=\sigma$, la quantité $\sigma>1$ devant se présenter souvent dans ce qui suivra.

IV. Cela posé, je dis que toutes les solutions de l'équation (1) sont données par la formule

$$
\text { (2) } t+u \vee D= \pm(\boldsymbol{T}+\boldsymbol{U} \boldsymbol{V} \boldsymbol{D})^{n}
$$

où il faut employer successivement chacun des deux signes et attribuer $\dot{a}$ l'exposant $n$ toutes les valeurs entières depuis $-\infty$ jusqu'à $\infty$, et de plus, que chaque solution est contenue d'une seule manière dans cette équation, c'est-à-dire qu'elle répond toujours à un signe et à un exposant déterminés. Il est sans doute inutile d'ajouter que pour faire usage de la formule (2), il faut égaler séparément les parties rationnelles et les coëfficients de $\boldsymbol{V} D$, après avoir développé le second membre, mis préalablement sous la forme $\pm(\boldsymbol{T}-\boldsymbol{U} \boldsymbol{V} \boldsymbol{D})^{-n}$, lorsque $n$ est négatif.

$1^{\circ}$. Il est d'abord facile de prouver que les entiers $t, u$ domnés par la formule (2), satisfont en effet à l'équation (1). Il suffit pour cela de remarquer que l'équation ( 2 ) subsiste également lorsqu'on y remplace $\checkmark D$ par $-\checkmark D$, et que l'équation ainsi modifiée, étant multipliée par l'équation primitive, donne précisément l'équation (1).

$2^{\circ}$. Pour faire voir en second lieu qu'il n'y a aucune solution de l'équation qui ne soit comprise dans la formule (2), posons pour un instant, $t_{n}+u_{n} \vee D=(T+U \vee D)^{n}$. L'équation (2) est alors équivalente $\dot{a}$ 
ces deux équations simultanées, $t= \pm t_{n}, u= \pm u_{n}$, les signes étant arbitraires, mais égaux dans les deux équations. Observons maintenant que, comme la puissance $(\boldsymbol{N}(\boldsymbol{T}+\boldsymbol{U} \boldsymbol{V} \boldsymbol{D}))^{n}=\sigma^{n}$ croît constamment depuis la valeur zéro jusqu'à une valeur infinie, lorsque l'exposant $n$ croît lui-même depuis $-\infty$ jusqu'à $\infty$, il faut nécessairement que relativement à une solution donnée $\tau, v$ quelconque, on ait

$$
\boldsymbol{N}(\tau+u \boldsymbol{V} \boldsymbol{D})=\sigma^{n}, \quad \text { ou } \sigma^{n}<\boldsymbol{N}(\tau+u \boldsymbol{V} \boldsymbol{D})<\sigma^{n+1},
$$

l'exposant $n$ ayant une valeur unique et déterminée. Dans le premier cas. où l'on a $N(\tau+u \sqrt{ } D)=N\left(t_{n}+u_{n} \vee D D\right)$, on conclura en vertu du $n^{\circ}$ II, $\tau= \pm t_{n}, v= \pm u_{n}$, où le signe qui doit être le même pour les deux équations, est complètement déterminé, l'entier donné $\tau$ ne pouvant se réduire à zéro. On voit donc que pour ce premier cas, la solution donnée $\tau, v$ est comprise dans l'équation ( 2 ) et répond $\dot{a}$ un exposant et $\dot{a}$ un signe entièrement déterminés. Reste à considérer la seconde hypothèse; la double inégalité qui s'y rapporte, étant divisée par $\sigma^{n}$, se change en celle-ci

$$
1<\frac{\boldsymbol{N}(\boldsymbol{x}+v \sqrt{ } \boldsymbol{D})}{\boldsymbol{N}\left(\boldsymbol{t}_{n}+u_{n} \sqrt{D}\right)}<\boldsymbol{N}(\boldsymbol{T}+\boldsymbol{U} \boldsymbol{V} \boldsymbol{D}),
$$

en vertu de laquelle la nouvelle solution $\tau^{\prime}$, $v^{\prime}$ donnée par la formule $\boldsymbol{\tau}^{\prime}+v^{\prime} \boldsymbol{V} \boldsymbol{D}=\frac{\boldsymbol{\tau}+\boldsymbol{v} \sqrt{\boldsymbol{D}}}{\boldsymbol{t}_{n}+u_{n} \sqrt{D}}$, satisferait à la condition $1<\boldsymbol{N}\left(\boldsymbol{\tau}^{\prime}+v^{\prime} \checkmark \boldsymbol{D}\right)<$ $\boldsymbol{N}(\boldsymbol{T}+\boldsymbol{U} \boldsymbol{V} \boldsymbol{D})$, ce qui est impossible, cette dernière inégalité étant en contradiction avec la définition du groupe fondamental. Le second cas ne pouvant avoir lieu, la proposition se trouvée établie.

V. L'équation (1) présente deux cas particuliers qui méritent une mention spéciale comme devant donner lieu plus tard à une application trèsremarquable; ces cas sont ceux où $\boldsymbol{D}$ est un entier réel ou le produit d'un tel entier par $i$. Comme dans la théorie des nombres complexes, l'équation (1) ne diffère pas essentiellement de celle où $D$ est remplacé par la valeur opposée, nous pouvons toujours considérer comme positif l'entier réel dont il vient d'être question.

$1^{\circ}$. Considérons en premier lieu le cas où $D$ est réel èt positif et supposons le radical $\boldsymbol{V D}$ également positif. Il est évident que, si dans le cas dont il s'agit on satisfait à l'équation (1) par les valeurs, $t=\alpha+\beta i$, $\boldsymbol{u}=\gamma+\delta i$, on y satisfera aussi par celles-ci, $t=\alpha-\beta i, u=\gamma-\delta i$. Or, ces deux solutions donnant évidemment la même valeur pour l'expression $\boldsymbol{N}(t+u \vee D)$, elles sont nécessairement identiques ou opposées, de 
sorte qu'on aura

$$
\alpha-\beta i= \pm(\alpha+\beta i), \quad \gamma-\delta i= \pm(\gamma+\delta i)
$$

et par suite ou $\beta=0, \delta=0$; ou $\alpha=0, \gamma=0$. On voit donc que $t$ et $u$ sont ou l'un et l'autre des entiers réels ou l'un et l'autre de tels entiers affectés $d u$ facteur $i$. Il résulte de là, et en ayant égard à la formule (2), que, si la solution fondamentale se trouve dans le premier cas, l'équation (1) n'a que des solutions réelles, tandis que pour une solution fondamentale imaginaire, les solutions de l'équation (1) sont en partie réelles, en partie imaginaires, les premières répondant $\dot{a}$ des valeurs paires et les dernières à des valeurs impaires de l'exposant; et si l'on observe que toute solution imaginaire de l'équation (1) donne sur le champ une solution réelle de celle-ci $t^{2}-D u^{2}=-1$, et réciproquement, on peut dire que la solution fondamentale présentera le second ou le premier des deux cas indiqués, suivant que l'équation $t^{2}-D u^{2}$ $=-1$, admet des solutions réelles ou non. Remarquons encore qu'en vertu de la condition $\boldsymbol{N}(\boldsymbol{T}+\boldsymbol{U} \boldsymbol{V} \boldsymbol{D})>1$ à laquelle la solution fondamentale est toujours supposée satisfaire, il est évident que dans le premier cas $\boldsymbol{T}$ et $\boldsymbol{U}$, et dans le second $T_{1}, U_{1}$ (en supposant $T=T_{1} i, U=U_{1} i$ ) sont toujours de même signe, de sorte que nous pourrons toujours considérer ces entiers comme positifs, l'inégalité précédente laissant le choix entre deux solutions fondamentales opposées. Cela posé, on voit que, si dans le premier cas on n'a en vue que les solutions positives de l'équation (1), il faudra dans la formule (2) adopter le signe supérieur et n'áttribuer à $n$ que des valeurs pareillement positives. La formule (2) ainsi restreinte donne évidemment des valeurs d'autant plus grandes pour le binome $t+u \checkmark D$, et par suite pour chacun des entiers $t$ et $u$, qui en vertu de l'équation (1) croissent toujours simultanément; que l'exposant $n$ est lui-même plus grand, d'où il suit que les deux termes de la solution fondamentale $T, U$ sont les plus petits entiers positifs qui résolvent l'équation (1). II serait également facile de faire voir que dans le second cas, $T_{1}, U_{1}$ sont les plus petits entiers positifs qui satisfont à l'équation $t^{2}-D u^{2}=-1$, mais il sera plus commode pour notre objet de n'employer que l'équation (1). Observons donc que pour obtenir toutes les solutions positives de cette dernière, il faudra après avoir posé dans la formule (2) $T=T_{1} i, U=U_{1} i$, remplacer $n$ par $2 n$ et supprimer ensuite la facteur $\pm(-1)^{n}$. On obtient ainsi $t+u \checkmark D$ $=\left(T_{1}+U_{1} \vee D\right)^{2 n}$, et l'on voit alors que la plus petite solution positive de l'équation (1) est donnée par la formule $t+u \checkmark D=\left(T_{1}+U_{1} \checkmark D\right)^{\text {. }}$. 
En désignant donc généralement par $\tau, u$ les plus petits entiers positifs qui résolvent l'équation (1), on aura suivant les deux cas

$$
\tau+u \vee \boldsymbol{D}=\boldsymbol{T}+\boldsymbol{U} \vee \boldsymbol{D}, \quad \tau+u \vee \boldsymbol{D}=\left(\frac{\boldsymbol{T}+\boldsymbol{U} \sqrt{D}}{i}\right)^{2} .
$$

Ces deux formules peuvent être réunies dans cette formule unique, dans laquelle $u$ désigne le nombre 1 ou le nombre 2, suivant que l'équation $t^{2}-D u^{2}=-1$, admet des solutions réelles ou n'en admet pas.

$$
\text { (3). } \sigma=\boldsymbol{N}(\boldsymbol{T}+\boldsymbol{U} \boldsymbol{V} \boldsymbol{D})=(\tau+v \boldsymbol{V D})^{x} \text {. }
$$

$2^{\circ}$. Pour traiter l'autre cas, soit $D=D^{\prime} i, D^{\prime}$ étant positif. Il est facile de voir que, si l'on satisfait alors à l'équation (1), en posant $t=$ $a+\beta i, \quad u=\gamma+\delta i$, on $y$ satisfera pareillement en posant $t=\alpha-\beta i$, $u=\delta+\gamma i$. Or, ces deux solutions donnant évidemment la même valeur pour l'expression $\boldsymbol{N}(\boldsymbol{t})+\boldsymbol{N}(\boldsymbol{u}) \boldsymbol{N}(\boldsymbol{V} \boldsymbol{D})$ qui, d'après ce qu'on a vu plus haut, peut servir à caractériser les différents groupes de solutions, on voit que les solutions précédentes appartiennent au même groupe. On a donc $\delta+\gamma i= \pm(\gamma+\delta i)$ et par conséquent $\delta= \pm \gamma$. Comme en vertu de ce résultat $u$ est toujours divisible par $1-i$, si nous posons dans l'équation (1), $u=(1-i) u^{\prime}, t=t^{\prime}$, elle prendra la forme $t^{\prime 2}-2 D^{\prime} u^{\prime 2}=1$, et nous retombons sur le cas déjà traité. Il est facile de conclure de là que l'expression $\sigma=\boldsymbol{N}(\boldsymbol{T}+\boldsymbol{U} \boldsymbol{V} \boldsymbol{D})$, toujours supposée supérieure à l'unité, est pour le cas dont nous nous occupons, donnée par l'équation

$$
\text { (4) } \sigma=\boldsymbol{N}(\boldsymbol{T}+\boldsymbol{U} \boldsymbol{V} \boldsymbol{D})=\left(\tau+\cup \sqrt{ } 2 \boldsymbol{D}^{\prime}\right)^{x} \text {, }
$$

$\tau$ et $v$ désignant les plus petits entiers positifs qui résolvent l'équation $t^{\prime 2}-2 \boldsymbol{D}^{\prime} u^{\prime 2}=1$, et $u$ ayant la valeur 1 ou la valeur 2 , suivant que l'équation $t^{\prime 2}-2 D^{\prime} u^{\prime 2}=-1$, admet des solutions réelles ou non.

\section{\$. 15.}

La théorie de l'équation $t^{2}-D u^{2}=1$, étant maintenant connue, nous pouvons reprendre les questions déja traitées plus haut et en achever la solution, en nous bornant, comme nous en avons déjà averti, à considérer des formes quadratiques qui appartiennent à la première espèce.

I. Nous nous occuperons en premier lieu de celle de ces questions, qui concerne les représentations d'un entier donné $m$ par une forme $(a, b, c)$ également donnée. Supposons que $m$ soit susceptible d'être représenté par la forme dont il s'agit, et soient $x=\alpha, y=\gamma$, des valeurs parti- 
culières et premières entre elles, telles que la valeur correspondante de la forme soit égale à $m$. Cela posé, il résulte du $\$ .12$, que toutes les représentations appartenant à la même valeur de l'expression $\boldsymbol{V D}$ (mod. $m$ ), à laquelle appartient la représentation particulière dont il s'agit, sont données par ces deux équations:

$$
x=a t-(b \alpha+c \gamma) u, \quad y=\gamma t+(a \alpha+b \gamma) u,
$$

où il faut substituer toutes les solutions de l'équation $t^{2}-D u^{2}=1$. Les équations précédentes, étant resp. multipliées par $a$ et $b+\checkmark D$, et ensuite ajoutées, donnent ce résultat très-simple

$$
a x+(b+\vee D) y=(a \alpha+(b+\vee D) \gamma)(t+u \checkmark D),
$$

qui au moyen de l'équation (2) du \$.14. se change en

$$
a x+(b+\sqrt{D}) y= \pm(a \alpha+(b+\boldsymbol{V} \boldsymbol{D}) \boldsymbol{\gamma})(\boldsymbol{T}+\boldsymbol{U} \boldsymbol{V} \boldsymbol{D})^{n} \text {. }
$$

Soit pour abréger, $\boldsymbol{N}(a \alpha+(b+\sqrt{D}) \gamma)=\boldsymbol{A}$, et comme dans le $\$$. cité, $\boldsymbol{N}(\boldsymbol{T}+\boldsymbol{U} \boldsymbol{V} \boldsymbol{D})=\sigma>1$. Cela posé, si l'on prend les normes des deux membres de l'équation précédente, on en conclura:

$$
\boldsymbol{N}(\boldsymbol{a} x+(\boldsymbol{b}+\boldsymbol{V} \boldsymbol{D}) \boldsymbol{y})=\boldsymbol{A} \sigma^{n},
$$

où il importe de remarquer que chaque valeur de $N(a x+(b+V D) y)$, donnée par cette dernière équation, se présentera deux fois dans la totalité des représentations que nous considérons et que pour abréger, nous nommerons désormais un groupe de représentations, comme cela résulte évidemment du double signe contenu dans l'équation précédente, et que le passage des nombres à leurs normes a fait disparaître. Observons encore que, si $k$ désigne une constante positive arbitrairement choisie, l'entier réel $n$ qui doit croître depuis - $\infty$ jusqu'à $\infty$, obtiendra évidemment une valeur et n'en obtiendra qu'une seule qui satisfasse à la double condition

$$
\boldsymbol{k}<\boldsymbol{A} \sigma^{n} \leqq k \sigma \text {. }
$$

On conclut de là que dans tout groupe de représentations; ou en d'autres termes, que parmi toutes les représentations qui appartiennent à une même valeur de l'expression $\boldsymbol{V} \boldsymbol{D}(\bmod . m)$, il en existe toujours deux et qu'il n'en existe que deux pour lesquelles on ait

$$
k<\boldsymbol{N}(a x+(b+v D) y) \leqq k \sigma ;
$$

et il est d'ailleurs manifeste qui les deux représentations particulières dont il s'agit et qui varient avec la constante $k$, sont toujours telles que, l'une étant exprimée par les formules: $x=r, y=s$, l'autre le sera par celles- 
ci: $x=-r, y=-s$. Le résultat que nous venons d'obtenir, va nous fournir un. moyen très-simple de décider $1^{\circ}$ si un entier donné $m$ peut être représenté par une forme également donnée $(a, b, c)$, ou non, et $2^{\circ}$ d'assigner dans le premier de ces deux cas, toutes les représentations dont $m$ est susceptible au moyen de la forme dont il s'agit. On voit d'abord que la question proposée revient à examiner si l'on peut satisfaire à ces deux conditions simultanées

$$
\begin{gathered}
\text { (1) } a x^{2}+2 b x y+c y^{2}=m, \\
\text { (2) } k<N(a x+(b+\vee D) y) \leqq k \sigma,
\end{gathered}
$$

par des entiers $x$ et $y$ premiers entre eux. Si cela n'est pas possible, on sera assuré que $m$ n'est pas susceptible d'être représenté par la forme donnée. Dans le cas contraire, on trouvera une ou plusieurs doubles représentations telles que $x= \pm r, y= \pm s ; x= \pm r^{\prime}, y= \pm s^{\prime}$; etc., qui appartiendront $\dot{a}$ autant de groupes distincts, et l'on obtiendra toutes les représentations cherchées, si dans les deux équations rappelées au commencement de ce $\$$., on pose successivement $\alpha=r, \gamma=s ; a=r^{\prime}, \gamma=s^{\prime}$; etc.

Réduite à ce point, la question ne présente plus aucune difficulté, car il est facile de se convaincre, que, pour que les entiers $x$ et $y$ puissent satisfaire aux conditions simultanées (1) et (2), leurs normes doivent être comprises entre certaines limites faciles $\dot{a}$ assigner, de sorte que l'examen qu'il s'agit de faire, ne doit porter que sur un nombre limité de combinaisons $x, y$. En effet, si après avoir multiplié par a l'équation (1), on prend les normes de ses deux membres, il viendra

$$
\boldsymbol{N}(\boldsymbol{a} x+(\boldsymbol{b}+\boldsymbol{r D}) \boldsymbol{y}) \boldsymbol{N}(\boldsymbol{a} x+(\boldsymbol{b}-\boldsymbol{V D}) \boldsymbol{y})=\boldsymbol{N}(\boldsymbol{a m}) .
$$

Cette équation étant comparée avec la double inégalité ( 2 ), on en conclura celle-ci

$$
\frac{N(a m)}{k \sigma} \leqq N(a x+(b-v D) y)<\frac{N(a m)}{k},
$$

et par suite en ajoutant cette dernière et l'inégalité (2),

$$
k+\frac{N(a m)}{k \sigma}<2 N(a x+b y)+2 \boldsymbol{N}(\boldsymbol{V D}) \boldsymbol{N}(\dot{y})<k \sigma+\frac{N(a m)}{k} .
$$

Il est facile de voir que les entiers $x$ et $y$, et à plus forte raison les entiers $x$ et $y$, premiers entre eux qui satisfont à cette double inégalité qui est une conséquence nécessaire des deux conditions (1) et (2), ne présentent qu'un nombre limité de combinaisons faciles a former, et l'on pourra douc tonjours décider 
si parmi ces entiers simultanés il en existe qui remplissent les deux conditions dont il s'agit, ce que nous nous étions proposé de faire voir.

La condition (2) qui, étant jointe à l'équation (1), a pour effet de réduire chaque groupe de représentations de l'entier $m$ par la forme $(a, b, c)$, à deux représentations particulières qu'elle sépare ainsi de toutes les autres, prend une forme remarquable lorsqu'on fait un choix convenable de la constante arbitraire $k$ qu'elle contient. Soit $k=N(\sqrt{ } a m)$. La condition dont nous parlons, devieudra ainsi

$$
\boldsymbol{N}(\boldsymbol{V} a m)<\boldsymbol{N}(\boldsymbol{a} x+(b+\boldsymbol{V} \boldsymbol{D}) \boldsymbol{y}) \leqq \sigma \boldsymbol{N}(\boldsymbol{V} a m) .
$$

Observons que, comme il ne s'agit que de quantités positives, cette double inégalité est tout-à-fait équivalente à celle-ci,

$$
\boldsymbol{N}(\boldsymbol{a} m)<(\boldsymbol{N}(\boldsymbol{a} \boldsymbol{x}+(\boldsymbol{b}+\boldsymbol{V} \boldsymbol{D}) \boldsymbol{y}))^{2} \leqq \sigma^{2} \boldsymbol{N}(\boldsymbol{a} m),
$$

qui $\dot{a}$ son tour peut être remplacée par celle qu'on en déduit en divisant par $\boldsymbol{N}(\boldsymbol{a} \boldsymbol{x}+(\boldsymbol{b}+\boldsymbol{V} \boldsymbol{D}) \boldsymbol{y})$, et en observant qu'on a

$$
\boldsymbol{N}(a m)=\boldsymbol{N}(a x+(b+\sqrt{ } \boldsymbol{D}) \boldsymbol{y}) \boldsymbol{N}(a x+(b-\checkmark D) y) .
$$

On trouve ainsi

(3) $\boldsymbol{N}(a x+(b-V D) y)<\boldsymbol{N}(a x+(b+\checkmark D) y) \leqq \sigma^{2} \boldsymbol{N}(a x+(b-\checkmark D) y)$.

C'est sous cette dernière forme que nous emploierons dorénavant la condition qui sert $\dot{a}$ réduire tout groupe de représentations à deux de ses termes.

11. Quant aux deux questions que nous nous étions proposées sur l'équivalence des formes, comme celle d'entre elles, qui a pour objet de déduire toutes les transformations d'une forme en une autre équivalente, d'une première transformation supposée donnée, s'est trouvée dépendre de l'équation $t^{2}-D u^{2}=1$, dont nous avons donné la solution générale, nous n'avons plus à traiter que la première des questions énoncées au commencement du \$. 11. Il s'agit donc de faire voir comment, étant données deux formes $(a, b, c)$ et $\left(a^{\prime}, b^{\prime}, c^{\prime}\right)$, ayant un déterminant commun $D$, on peut décider si ces formes sont équivalentes ou non, et obtenir dans le prenier de ces deux cas, l'une des substitutions au moyen desquelles la première se change dans la seconde. Pour résoudre cette question, on se rappellera que, d'après ce qu'on a démontré dans le \$.12, tout revient à voir s'il existe des représentations de l'entier $a^{\prime}$ par la forme $(a, b, c)$, qui appartiennent à la valeur $b^{\prime}$ de l'expression $\boldsymbol{V} \boldsymbol{D}$ (mod. $\left.a^{\prime}\right)$. Si l'on trouve qu'il n'y a aucune représentation pour laquelle la condition énoncée soit 
satisfaite, on sera assuré que les deux formes ne sont pas équivalentes; dans le cas contraire, l'une quelconque des représentations obtenues donnera sur le champ la transformation cherchée. La question proposée se trouvant ainsi réduite à celle dont nous avons donné la solution dans le $\mathbf{n}^{\circ}$ précédent de ce $\$$., doit elle-même être considérée comme résolue.

III. Avant d'en venir à la question qui forme le principal sujet de ce Mémoire, nous avons encore a indiquer, comment, étant donnée une forme $a x^{2}+2 b x y+c y^{2}$ du déterminant $D$, on peut assigner d'une manière générale les valeurs simultanées $x$ et $y$, pour lesquetles la valeur de l'expression précédente soit impaire et première à $D$, ou plus simplement, soit première à $(1+i) \boldsymbol{D}=\Delta$. Comme, en posant $x \equiv \alpha, y \equiv \gamma,(\bmod . \Delta)$, où $\alpha$ et $\gamma$ peuvent être choisis dans un système de résidus relatif au module $\Delta$, l'on a

$$
a x^{2}+2 b x y+c y^{2} \equiv a \alpha^{2}+2 b \alpha y+c \gamma^{2}(\bmod . \Delta),
$$

on voit que la question proposée se réduit à examiner pour lesquelles des combinaisons $\alpha, \gamma$, ou plutôt pour combien de ces combinaisons, car c'est uniquement leur nombre qu'il nous importe de connaître, le second membre est premier à $\Delta$. J'observe maintenant que sans nuire en rien à la généralité de la question, il est permis de considérer le coëfficient $a$ comme premier à 4 . En effet, comme la forme donnéé est supposée de première espèce, on peut toujours, si elle ne satisfait pas à la condition énoncée, la transformer en une autre où cette dernière se trouve remplie; et l'on prouve facilement que relativement à la nouvelle forme, le nombre des combinaisons dont il s'agit, est le même que pour la forme donvée. Le raisonnement par lequel cette dernière assertion peut être justifiée, étant trèssimple et d'ailleurs entièrement semblable à celui dont nous avons déjà fait usage dans la question analogue, relative aux entiers réels, nous nous dispenserons de le répéter ici. (R. s. d. a. etc. \$.5.)

Cela posé, il est évident que, pour que l'expression $a \alpha^{2}+2 b a \gamma+c \gamma^{2}$ soit première $\dot{a} A=(1+i) D$, il faut et il suffit qu'il en soit de mème du produit

$$
\boldsymbol{a}\left(\boldsymbol{a} \alpha^{2}+2 b \alpha \gamma+c \gamma^{2}\right)=(\boldsymbol{a} \alpha+b \gamma)^{2}-\boldsymbol{D} \gamma^{2} \text {. }
$$

Distinguons maintenant le cas où $\boldsymbol{D}$ est impair et celui où $\boldsymbol{D}$ est divisible par $1+i$. Dans le prenier de ces deux cas, il faudra, si $\gamma$ est divisible par $1+i$, que $a c+b y$ soit premier $\dot{a} \Delta$, et, si $\gamma$ est impair, que $a a+b y$ soit divisible par $1+i$ et premier à $D$. Or, comme, $\gamma$ ayant une valeur 
déterminée, l'expression $a \alpha+b \gamma$, dans laquelle $\alpha$ est le terme géuéral d'un système de résidus pour le module 4 , représente elle-même un semblable système (\$. 5, III.), il s'agira de déterminer combien dans un système de résidus pour le module $\Delta$, il existe de termes premiers $\dot{a} \Delta$ ou de termes premiers à $D$ et en outre divisibles par $1+i$, selon que $\gamma$ sera ou ne sera pas divisible par $1+i$. Le premier de ces deux nombres est $\psi(\Delta)$; poúr obtenir le second, on se rappellera que, si l'on divise par $1+i$, ceux des termes du système en question, qui renferment le facteur $1+i$, les quotients formeront un système de résidus pour le module $D(\$ .5$, II.), d'où l'on conclut que le nombre que nous avons à déterminer, est exprimé par $\psi(D)$. J'ajoute que cette dernière expression peut être remplacée par $\psi(\Delta)$, puisque, $D$ et $1+i$ étant premiers entre eux, on a $(\$ .5, V),. \psi(\Delta)=$ $\psi((1+i) D)=\psi(1+i) \psi(\boldsymbol{D})=\psi(\boldsymbol{D})$. Ayant ainsi reconnu qu'à toute valeur déterminée $\gamma$ il répond un nombre $\psi(\Delta)$ de valeurs $\alpha$, qui satisfont aux conditions exigées, et sachant d'un autre côté que $\gamma$ est susceptible d'un nombre de valeurs, exprimé par $\boldsymbol{N}(\Delta)$, on en conclura que les combinaisons $\alpha, \gamma$, qui rendent $a \alpha^{2}+2 b a \gamma+c \gamma^{2}$ premier $\dot{a} \Delta$, sont au nombre de $\boldsymbol{N}(\Delta) \psi(\Delta)$.

Le cas où $D$ est supposé divisible par $1+i$, donne le même résultat. En effet, comme le terme $D \gamma^{2}$ est dans ce cas divisible par $1+i$, tout se réduit à faire en sorte que $a \alpha+b \gamma$ soit premier $\grave{a} \Delta$, et l'on voit facilement que les valeurs $\alpha$ qui, répondant à une valeur déterminée $\gamma$, satisfont à cette condition, sont toujours au nombre de $\psi(\Delta)$, d'où l'on conclut que celui des combinaisons dont il s'agit, est égal $\dot{a} \boldsymbol{N}(\Delta) \psi(\Delta)$, comme dans le premier cas.

On voit ainsi que les valeurs simultanées de $x$ et de $y$, qui, étant substituées dans l'expression $a x^{2}+2 b x y+c y^{2}$, la rendent première à $(1+i) \boldsymbol{D}=\Delta$, peuvent toujours être distribuées en systèmes de la forme,

$$
x=\Delta v+\alpha, \quad y=\Delta w+\gamma,
$$

oú $v$ et $w$ sont des entiers indéterminés, et $\alpha$ et $\gamma$ des entiers déterminés; et que le nombre de ces systèmes est toujours exprimé par $N(\Delta) \psi(\Delta)$. 
Classification des formes et théorèmes qui s'y rapportent.

ร. 16.

La classification dont il s'agit, consiste à rapporter deux quelconques des formes qui ont un déterminant commun $D$, à la même classe ou à des classes distinctes, suivant que ces formes sont équivalentes ou non. Nous démontrerons d'abord que les classes ainsi définies sont toujours en nombre limité, quel que soit le déterminant donné. C'est à quoi nous parviendrons, en faisant voir que dans chaque classe il existe au moins une forme $(a, b, c)$, telle qu'on ait à la fois $\frac{1}{2} \mathbf{N}(a) \geqq \mathbf{N}(b), \quad \boldsymbol{N}(a) \leqq N(c)$, et en prouvant ensuite que les formes de cette nature, qu'on appelle des formes réduites, sont toujours en nombre fini. Pour établir le premier de ces deux points, il s'agira de montrer qu'une forme quelconque $\left(a, b, a^{\prime}\right)$ peut toujours se transformer en une forme réduite équivaleute. Considérons à cet effet la substitution $\left(\begin{array}{l}\alpha, \beta \\ \gamma, \delta\end{array}\right)$, où nous n'avons que cette seule condition $a \delta-\beta \gamma=1$, et observons que cette dernière sera satisfaite, si, $\delta$ restant quelconque, nous supposons $\alpha=0, \beta=1, \gamma=-1$. Au moyen de la substitution ainsi particularisée, la forme $\left(a, b, a^{\prime}\right)$ se changera en une autre telle que $\left(a^{\prime}, b^{\prime}, a^{\prime \prime}\right)$, où l'on aura $b^{\prime}=-b-a^{\prime} \delta$. D'après ce nous avons remarqué au commencement du. $\$$. 2 , nous pouvons toujours disposer de l'indéterminée $\delta$ de manière à ce que l'on ait $\frac{1}{2} N\left(a^{\prime}\right) \geqq N\left(b^{\prime}\right)$. La nouvelle forme $\left(a^{\prime}, b^{\prime}, a^{\prime \prime}\right)$ satisfaisant alors à la première des deux conditions qui définissent les formes réduites, si l'on a en outre $N\left(a^{\prime}\right) \leqq N\left(a^{\prime \prime}\right)$, cette forme aura toutes les propriétés requises; si non, on en déduira par le même procédé une troisième forme telle que $\left(a^{\prime \prime}, b^{\prime \prime}, a^{\prime \prime \prime}\right)$, où l'on aura $\frac{1}{2} \boldsymbol{N}\left(a^{\prime \prime}\right) \geqq \mathbf{N}\left(b^{\prime \prime}\right)$, et qui par conséquent sera une forme réduite si l'on a en outre $\boldsymbol{N}\left(a^{\prime \prime}\right) \leqq N\left(a^{\prime \prime \prime}\right)$. Il est manifeste que, si l'on continue à opérer toujours de la même manière, on finira nécessairement par tomber sur uue forme réduite équivalente à la proposée; car pour qu'il en fût autrement, il faudrait que la suite $N\left(a^{\prime}\right)>N\left(a^{\prime \prime}\right)>N\left(a^{\prime \prime \prime}\right)>$ etc. pût être indéfiniment prolongée, ce qui évidemment est impossible, les entiers $a^{\prime}$, $a^{\prime \prime}, a^{\prime \prime}, \ldots$ étant tous différents de zéro, si, comme on le suppose tou-. jours, $D$ n'est pas un quarré. Le premier point se trouvant ainsi établi, il nous reste à faire voir que les formes réduites $(a, b, c)$ qui ont un déterminant donné $D$, sont en nombre limité et peuvent toujours être assignées 
facilement. Les deux conditions $\frac{1}{2} \boldsymbol{N}(a) \geq \boldsymbol{N}(b), \quad \boldsymbol{N}(a) \leqq N(c)$, donnent d'abord $\boldsymbol{N}(a c) \geq 4 \boldsymbol{N}\left(b^{2}\right)$, et par suite $\boldsymbol{V N}(a c) \geqq 2 N(b)$. Si d'un autre côté, on applique à l'équation $a c=b^{2}-D$, le théorème déjà employé dans le \$.13, on en conclura $\checkmark \boldsymbol{N}(a c) \leqq \checkmark N\left(b^{2}\right)+\checkmark N(-D)$, ou ce qui revient au même, $\boldsymbol{V} \boldsymbol{N}(a c) \leqq N(b)+\checkmark \boldsymbol{N}(\boldsymbol{D})$, inégalité qu'il suffit de comparer à celle déjá obtenue, pour voir qu'on a $\boldsymbol{N}(\boldsymbol{b}) \leqq \checkmark \boldsymbol{N}(\boldsymbol{D})$. Comme $\boldsymbol{N}(\boldsymbol{b})$ et par conséquent aussi $\boldsymbol{b}$ n'est ainsi susceptible que d'un nombre limité de valeurs faciles à assigner, pour obtenir toutes les formes réduites du déterminant $D$, il suffira de décomposer chacune des valeurs correspondantes de $b^{2}-D$, de toutes les manières possibles en deux facteurs $a$ et $c$, en supposant $\boldsymbol{N}(\boldsymbol{a}) \leqq \boldsymbol{N}(\boldsymbol{c})$, et de ne conserver que celles des combinaisons $a, b, c$, pour lesquelles on a $\left.\frac{1}{2} \boldsymbol{N}(a) \geqq N(b) *\right)$.

Ayant ainsi.obtenu toutes les formes réduites $(a, b, c)$ qui répondent à un déterminant donné, si, comme nous le supposons, on n'a en vue que les formes qui appartiennent à la première espèce, il ne restera plus qu'à effacer celles des formes trouvées, pour lesquelles $a, b, c$ ou $a, 2 b, c$ auraient un diviseur commun.

Il s'agit maintenant de faire l'énumération complète des classes qui répondent au déterminant $D$, en choisissant daus chacune de ces classes l'une quelconque des formes dont elle se compose. Des formes choisies d'après cette règle, constitueront ce que nous appellerous un système complet de formes non-équivalentes ou plus simplement, un systeme de formes pour le déterminant dont il s'agit. Un tel système jouira évidemment de la double propriété de présenter une forme et de n'en présenter qu'une seule qui soit équivalente à une forme quelconque, pourvu que cette dernière ait l'entier $\boldsymbol{D}$ pour déterminant et soit d'ailleurs de première espèce. Pour construire un système de cette nature, on peut se servir des formes réduites que nous avons appris à déterminer dans ce qui précède. En effet, comme parmi les formes réduites il s'en trouve toujours au moins une, qui appartieune $\dot{a}$ une classe arbitrairement choisie, tout reviendra à éliminer les formes surabondantes. Après avoir rangé à cet effet les formes ré-

*) On voit que la méthode dont nous venons de faire usage pour obtenir les formes réduites, est entièrement analogue à celle qui sert pour le mème objet dans la théorie des entiers réels. Nous ajouterons que la possibilité d'appliquer cette dernière aux entiers complexes, avait déjà été remarquée par Mr. Jacobi. Tome XIX, page 314 de ce Journal. 
duites dans un ordre quelconque, on commencera par comparer la première d'entre elles à chacune des suivantes et l'on effacera toutes celles de ces' dernières, que l'on reconnaîtra lui être équivalentes. Cela fait, on comparera la seconde des formes que cette première opération aura laissées subsister, ’̀ chacune des suivantes pour effacer encore les formes qu'on trouvera lui être équivalentes, et ainsi de suite.

Le procédé que nous venons d'indiquer, suffit pour assigner le nombre des classes, ou ce qui revient au même, celui des termes composant un système de formes pour un déterminant quelconque, lorsque ce dernier est numériquement donné. Mais tel n'èst pas l'objet principal que nous nous sommes proposé dans ce Mémoire, et qui consiste plutôt à découvrir la loi générale par laquelle le nombre des classes se trouve lié au déterminant auquel ces classes se rapportent. Pour résoudre cette dernière question, il faut pénétrer plus avant dans la nature de ce que nous avons nommé un système de formes, et se rendre compte des rapports qui existent entre un tel assemblage et la totalité des entiers que ces formes peuvent servir à représenter.

\section{\$. 17.}

Soit

(1) $\quad a x^{2}+2 b x y+c y^{2}, \quad a^{\prime} x^{2}+2 b^{\prime} x y+c^{\prime} y^{2}, \ldots$

un système de formes (de première espèce) pour le déterminant $D$, et proposons-nous de rechercher sous quelles conditions un entier $m$ que nous supposerons impair et premier $\dot{a} \boldsymbol{D}$, ou réunissant ces denx conditións en une seule, que nous supposerons premier à $\Delta=(1+i) D$, peut être représenté par une ou par plusieurs de ces formes, et de déterminer, lorsque de telles représentations existent, le nombre des groupes dans lesquels leur totalité se distribue. Il est bien entendu que, comme dans ce qui précède, il n'est toujours question que de représentations pour lesquelles les indéterminées $x$ et $y$ soient premières entre elles. D'après le $\$ .12$, il y a une première condition à remplir, consistant en ce que $D$ doit être résidu quadratique à l'égard de $m$, et il résulte d'un autre côté du $\$ .9$, que, pour qu'elle soit satisfaite, il faut et il suffit que pour chacun des diviseurs simples $f$ de $m$, on ait

$$
\text { (2) }\left[\frac{D}{f}\right]=1
$$


Ces conditions particulières étant supposées remplies, si l'on désigne par $\mu$ le nombre des facteurs simples primaires inégaux que l'entier $m$ contient, la congruence $z^{2} \equiv \boldsymbol{D}$ (mod. $m$ ), aura $2^{\mu}$ racines et il s'agira de chercher quels sont les groupes de représentations qui puissent répondre à ces diverses racines. Soit $l$ l'une quelconque de ces racines, et proposons-nous de déterminer les représentations qui y appartiennent. D'après ce qui a été demontré dans le $\$ .12$, nous avons à examiner si parmi les formes (1) il y en une qui soit équivalente à celle-ci $\left(m, l, \frac{l^{2}-D}{m}\right)$. Observons d'abord que les coëfficients de cette dernière sont évidemment sans diviseur commun, puisqu'un tel diviseur diviserait aussi le déterminant $D$, ce qui serait contraire à l'hypothèse admise d'après laquelle $m$ est premier à $D$. Comme d'un autre côté, $m$ est supposé impair, on voit que la forme précédente appartient à la première espèce, d'où il suit que la forme dont il s'agit, a son équivalente dans le système (1). Il résulte de là et du $\mathbf{5}$. déjà cité, qu'il existe toujours un groupe de représentation appartenant à une racine déterminée $l$, et qu'il n'en existe qu'un seul, d'où l'on conclut que les représentations dont l'entier $m$ est susceptible au moyen des expressions (1), forment toujours un nombre de groupes distincts, égal à la puissance $2^{\mu}$.

Nous pouvons maintenant réduire chacun des groupes dont il s'agit, à deux représentation individuelles, si dans chacune des formes (1), nous limitons les indéterminées $x$ et $y$, an moyen de la condition d'inégalité déjà donnée dans le $\$$. 15, cette condition étant pour la première des expressions (1),

$$
\boldsymbol{N}(a x+(b-v D) y)<\boldsymbol{N}(a x+(b+\checkmark D) y) \leqq \sigma^{2} \boldsymbol{N}(a x+(b-v D) y),
$$

et se déduisant pour les autres de celle que nous venons d'écrire, en y accentuant les lettres $a, b, c$. Ces conditions étant jointes aux formes (1), on voit que le nombre des représentations dont $m$ est susceptible au moyen des formes dont il s'agit, sera fini et exprimé par $2^{\mu+1}$.

Au moyen du résultat que nous venons d'obtenir, il est facile de former l'équation générale que nous allons écrire:

$$
\begin{gathered}
\text { (4) } \quad \sum 2^{\mu+1} \boldsymbol{F}(\boldsymbol{m})= \\
\sum \boldsymbol{F}\left(\boldsymbol{a} \boldsymbol{x}^{2}+2 \boldsymbol{b} \boldsymbol{x} y+c y^{2}\right)+\sum \boldsymbol{F}\left(\boldsymbol{a}^{\prime} \boldsymbol{x}^{2}+2 \boldsymbol{b}^{\prime} x y+\boldsymbol{c}^{\prime} y^{2}\right)+\text { etc. }
\end{gathered}
$$

La sommation indiquée dans le premier membre est supposée embrasser la totalité des entiers $m$ premiers à $\Delta$, dont tous les diviseurs simples $f$ satisfont à la condition ( 2 ), $\mu$ désignant pour chacun de ces entiers $m$, le 
nombre de ses facteurs simples primaires inégaux. Quant aux sommes contenues dans le second membre, elles sont en même nombre que les formes (1), et répondent chacune à l'une des formes en question. Dans chacune de ces sommes le signe $\Sigma$ doit s'étendre a tous les systèmes de valeurs simultauées $x$ et $y$, qui remplissent la triple condition de n'avoir pas de diviseur commun, de douner à la forme où elles sont substituées, une valeur première à $A$, et enfin de satisfaire à la double inégalité (3), lorsqu'il s'agit de la pmemière somme et à des inégalités de même forme pour chacune des autres. La fonction désignée par la caractéristique $\boldsymbol{F}$ est arbitraire et doit seulement être choisie de manière à ce que les séries contenues dans l'équation, soient convergentes et aient des sommes indépendantes de l'ordre de succession de leurs termes. La vérité de l'équation ainsi formée est évidente, et l'on voit que cette équation n'est que la traduction de la double propriété remarquée plus haut et consistant en ce que d'une part, tout entier supposé premier à $\Delta$, pour être susceptible d'être représenté par les formes (1), doit être compris parmi ceux que nous venons de désigner par $m$; et en ce que d'autre part, chacun de ces derniers admet en effet $2^{\mu+1}$ représentations au moyen des expressions (1), si à chacune de ces expressions l'on suppose jointe une condition d'iuégalité comme celle (3). D'après la manière dont l'équation précédente subsiste, il est manifeste qu'elle ne cessera pas d'avoir lieu, si l'on y remplace partout les entiers complexes qui se trouvent sous Je signe $F$, par leurs normes, de sorte qu'on aura aussi

$$
\begin{gathered}
\sum 2^{\mu+1} \boldsymbol{F}(\boldsymbol{N} \boldsymbol{m})= \\
\Sigma \boldsymbol{F}\left(\boldsymbol{N}\left(\boldsymbol{a} x^{2}+2 b x y+c y^{2}\right)\right)+\sum \boldsymbol{F}\left(\boldsymbol{N}\left(\boldsymbol{a}^{\prime} x^{2}+2 \boldsymbol{b}^{\prime} x y+c^{\prime} y^{2}\right)\right)+\text { etc. }
\end{gathered}
$$

les signes sommatoires ayant toujours la même signification. Particularisons la fonction arbitraire contenue dans l'équation, et supposons que cette fonction soit une puissance de l'exposant $-s$, où $s$ est une quantité positive supérieure à l'unité. Il viendra ainsi en n'écrivant, pour abréger, que le premier terme du second membre,

$$
\sum \frac{2^{\mu+1}}{(\bar{N} m)^{s}}=\Sigma \frac{1}{\left(\boldsymbol{N}\left(a x^{2}+2 b x y+c y^{2}\right)\right)^{s}}+\text { etc. }
$$

Comıne d'après la définition des entiers $m$, quatre nombre associés se trouvent évidemment toujours simultauément compris ou uon parmi ces entiers $m$, on voit que nous pouvons considérer la sommation indiquéc dans le premier membre, comme ne devant plus s'étendre qu'aux entiers on qui 
satisfaisant aux conditions énoncées plus haut, soient en outre primaires, c'est-à-dire tels qu'en posant $m=\alpha+\beta i$, on ait $\alpha \equiv 1(\bmod .4), \beta \equiv 0$ (mod. 2), pourvu qu'en même temps nous quadruplions le premier membre. On aura ainsi

$$
\text { (5) } 8 \Sigma \frac{2^{\mu}}{(N m)^{s}}=\Sigma \frac{1}{\left(N\left(a x^{2}+2 b x y+c y^{2}\right)\right)^{s}}+\text { etc. }
$$

L'entier $m$ étant primaire, on aura toujours d'une manière unique $(\$ \$ .2$ et 3, V.), $m=f^{\prime h^{\prime}} f^{\prime \prime h^{\prime \prime}} \ldots$, les exposants $h^{\prime}, h^{\prime \prime}, \ldots$ étant tous différents de zéro, et $f^{\prime}, f^{\prime \prime}, \ldots$ désiguant des nombres premiers primaires inégaux, qui satisfont à la condition ( 2 ), et dont le nombre est exprimé par $\mu$. Cela étant, il est facile de se convaincre qu'on a

$$
\text { (6) } \Sigma \frac{2^{\mu}}{(\boldsymbol{N} m)^{s}}=\Pi \frac{1+\frac{1}{(\boldsymbol{N} f)^{s}}}{1-\frac{1}{(\boldsymbol{N} \boldsymbol{f})^{s}}}
$$

le signe $\Pi$ s'étendant à tous les nombres premiers impairs et primaires $f$, qui ne divisent pas le déterminant $D$ et remplissent la condition ( 2 ). Il suffit de dévèlopper le facteur général comme il suit:

$$
\begin{aligned}
\frac{1+\frac{1}{(N \boldsymbol{f})^{s}}}{1-\frac{1}{(\boldsymbol{N} f)^{s}}} & =1+\frac{2}{(\boldsymbol{N} f)^{s}}+\frac{2}{(\boldsymbol{N} f)^{2 s}}+\frac{2}{(\boldsymbol{N} f)^{3 s}}+\text { etc. } \\
& =1+\frac{2}{(\boldsymbol{N} f)^{s}}+\frac{2}{\left(N \cdot f^{2}\right)^{s}}+\frac{2}{\left(N \cdot f^{3}\right)^{s}}+\text { etc. }
\end{aligned}
$$

et d'effectuer ensuite la multiplication, pour reconnaître au moyen de la remarque que nous venons de faire, que l'équation précédente est ell effet exacte. Pour transformer ultérieurement le second membre de cette équation, soit $q$ le terme général de la suile des nombres premiers impairs et primaires, à l'exclusion de ceux qui divisent $D$, et considérons le produit

$$
\Pi \frac{1}{1-\frac{1}{(N q)^{s}}}
$$

où le signe de multiplication est supposé s'étendre à toutes les valeurs y que nous venons de défiuir. Comme l'on a

$$
\frac{1}{1-\frac{1}{(N q)^{s}}}=1+\frac{1}{(N q)^{s}}+\frac{1}{\left(N \cdot q^{2}\right)^{s}}+\frac{1}{\left(N \cdot q^{s}\right)^{s}}+\text { etc. }
$$

et qu'on sait d'un autre côté, que tout entier impair et primaire n'est sus- 
ceptible que d'une seule décomposition en facteurs simples également primaires, l'on voit que le produit précédent équivaut à une série d'une loi très-śimple, et que l'on a

$$
\text { (7) } \quad \text { II } \frac{1}{1-\frac{1}{(N q)^{s}}}=\Sigma \frac{1}{(N n)^{s}} \text {, }
$$

le signe $\Sigma$ se rapportant à tous les entiers impairs $n$, primaires et premiers à $D$. Si au lieu du produit précédent, l'on considère le suivant

$$
\Pi \frac{1}{1-\left[\frac{D}{q}\right] \frac{1}{(N q)^{s}}}
$$

on reconnaîtra que ce nouveau produit, traité de la même manière, se transforme en une série ayant pour terme général $\chi \frac{1}{(N n)^{s}}$, où le coëfficient $\chi$ sera donné par la formule $\chi=\left[\frac{D}{q^{\prime}}\right]^{h^{\prime}}\left[\frac{D}{q^{\prime \prime}}\right]^{h^{\prime \prime}} \ldots$, si l'on suppose $n=q^{\prime h^{\prime}} \boldsymbol{q}^{\prime h^{\prime \prime}} \ldots$. les exposants étant positifs, et $\dot{q}^{\prime}, \boldsymbol{q}^{\prime \prime}, \ldots$ désignant les nombres premiers inégaux $q$ que l'entier $n$ contient. Si maintenant l'on observe qu'en vertu de la troisième des équations $(e)$ du $\$ .8$, l'expression $\chi$ peut être remplacée par

$$
\left[\frac{D}{q^{\prime h^{\prime}}}\right]\left[\frac{D}{q^{\prime h^{\prime \prime}}}\right] \ldots=\left[\frac{D}{q^{\prime h^{\prime}} q^{\prime \prime h^{\prime \prime}} \ldots}\right]=\left[\frac{D}{n}\right],
$$

on aura l'équation

$$
\text { (8) } \Pi \frac{1}{1-\left[\frac{D}{q}\right]\left[\frac{1}{(N q)^{s}}\right]}=\Sigma \cdot\left[\frac{D}{n}\right] \frac{1}{(N n)^{s}} \text {, }
$$

dans laquelle les signes $\Pi$ et $\Sigma$ ont la même signification que dans l'équation (7). Cela posé, faisons le produit des équations (7) et (8), et divisons ensuite ce produit pàr l'équation (7), après avoir remplacé dans cette dernière $s$ par $2 s$. Le facteur général du premier membre de l'équation que l'on obtient ainsi, sera évidemment

$$
\frac{1-\frac{1}{(N q)^{2 s}}}{\left(1-\frac{1}{(N q)^{s}}\right)\left(1-\left[\frac{D}{q}\right] \frac{1}{(N q)^{s}}\right)}=\frac{1+\frac{1}{(N q)^{s}}}{1-\left[\frac{D}{q}\right] \frac{1}{(N q)^{s}}}
$$

Ce facteur présente deux cas différents selon que l'on a $\left(\frac{D}{q}\right)=1$, ou $\left[\frac{D}{q}\right]$ $=-1$. Dans le second il se réduit à l'unité et peut être omis, tandis 
que pour le premier il prend la forme

$$
\frac{1+\frac{1}{(N q)^{s}}}{1-\frac{1}{(N q)^{s}}}
$$

Or, les nombres premiers $q$ pour lesquels on a $\left[\frac{D}{q}\right]=1$, coincidant avec ceux que nous avons précédemment désignés par $f$, on voit que l'équation qu'il s'agit de former, est:

$$
\Pi \frac{1+\frac{1}{(\boldsymbol{N} f)^{s}}}{1-\frac{1}{(N f)^{s}}}=\frac{\Sigma \frac{1}{(\boldsymbol{N} n)^{s}} \cdot \Sigma\left[\frac{D}{n}\right] \frac{1}{(\boldsymbol{N} n)^{s}}}{\Sigma \frac{1}{(\boldsymbol{N} n)^{2 s}}} .
$$

Au moyen de ce résultat et de l'équation (6), l'équation (5) peut prendre la forme:

$$
8 \Sigma \frac{1}{(N n)^{s}} \cdot \Sigma\left[\frac{D}{n}\right]_{\frac{1}{(N n)^{s}}}=\Sigma \frac{1}{\left(N \cdot n^{2}\right)^{s}} \cdot \Sigma \frac{1}{\left(N\left(a x^{2}+2 b x y+c y^{2}\right)\right)^{s}}+\text { etc. }
$$

où les signes sommatoires qui se rapportent $\dot{a} n$, s'étendent $\dot{a}$ tous les entiers primaires et premiers $\dot{a} A$, tandis que ceux relatifs aux valeurs simultanées $x$ et $y$, conservent la signification indiquée plus haut. Il est facile de se convaincre que les produits de séries, contenus dans le second membre, sont susceptibles d'une forme beaucoup plus simple qu'ils prennent lorsque la multiplication indiquée est effectuée. Pour leur donner cette nouvelle forme, nous considérerons particulièrement le premier de ces produits, la même transformation s'appliquant à tous les autres. En faisant le produit des termes généraux des deux sommes qu'il s'agit de multiplier entre elles, on aura:

$$
\left.\left.\frac{1}{\left(N \cdot n^{2}\right)^{s}\left(N\left(a x^{2}+2 b x y+c y^{2}\right)\right)^{s}}=\frac{1}{\left(N \left(a x^{\prime 2}+2\right.\right.} \bar{b} x^{\prime} y+c y^{\prime 2}\right)\right)^{s},
$$

où l'on a posé, $x^{\prime}=n x, y^{\prime}=n y$. Voyons quelle est la nature des systèmes $x^{\prime}, y^{\prime}$, auxquels la nouvelle sommation doit se rapporter. Comme l'on a $n^{2}\left(a x^{2}+2 b x y+c y^{2}\right)=a x^{\prime 2}+2 b x^{\prime} y^{\prime}+c y^{\prime 2}$, on voit d'abord, en ayant égard aux conditions que $x, y, n$, sont supposés remplir, $1^{\circ}$ que pour chacun des systèmes en question, $a x^{\prime 2}+2 b x^{\prime} y+c y^{\prime 2}$ est premier $\dot{\mathbf{a}} A$, et $2^{\circ}$ que les entiers $x^{\prime}, y^{\prime}$ satisfont à la double inégalité $\boldsymbol{N}\left(a x^{\prime}+(b-v \boldsymbol{D}) y^{\prime}\right)<\boldsymbol{N}\left(a x^{\prime}+(b+\checkmark D) y^{\prime}\right) \leqq \sigma^{2} \boldsymbol{N}\left(a x^{\prime}+(b-\checkmark D) \dot{y}^{\prime}\right)$ de même forme que celle (3), et qui résulte de cette dernière en multipliant Crelle's Journal f. d. M. Bd. XXIV. Heft 4. 
$\operatorname{par} N(n)$. Il est facile de prouver réciproquement, que tout système $x^{\prime}, y^{\prime}$, qui satisfait à ces deux conditions, est en effet compris parmi ceux auxquels la nouvelle sommation doit s'étendre et ne s'y présente qu'une fois. C'est $\dot{a}$ quoi l'on parvient, en assignant l'entier $n$ et le système $x, y$, l'un et l'autre entièrement déterninés, de la combinaison desquels le système donné $x^{\prime}, y^{\prime}$ provient. Soit à cet effet, $x^{\prime}=n x, y^{\prime}=n x$, où $n$ désigne le plus grand diviseur commun primaire de $x$ et $y$, qui sera complètement déterminé ainsi que les entiers $x$ et $y$. Cela étant, il est évident que $n$ est premier à $A$, et l'on voit également sans difficulté que les entiers $x$ et $y$, premiers entre eux, satisfont aussi aux deux autres conditions auxquelles les systèmes $x, y$ sont assujettis. Cela est manifeste pour celle de ces conditions, qui consiste en ce que $a x^{2}+2 b x y+c y^{2}$ doit être premier à $A$, et pour prouver que la double inégalité (3) a pareillement lieu, il suffit de diviser par $\boldsymbol{N}(\boldsymbol{n})$, celle écrite plus haut et à laquelle $x^{\prime}$ et $\boldsymbol{y}^{\prime}$ sont supposés satisfaire.

Après avoir ainsi reconnu la nature des systèmes $x^{\prime}, y^{\prime}$, que la nouvelle sommation doit embrasser, nous pouvons supprimer les accents des indéterminées $x^{\prime}$ et $y^{\prime}$. L'équation qu'il s'agissait de transformer, deviendra ainsi:

$$
8 \Sigma \frac{1}{(N n)^{s}} \cdot \Sigma\left[\frac{D}{n}\right] \frac{1}{(N n)^{s}}=\Sigma \frac{1}{\left(N\left(a x^{2}+2 b x y+c y^{2}\right)\right)^{s}}+\text { etc. }
$$

où la double sommation indiquée dans le premier terme du second membre est supposée s'étendre aux valeurs simultanées $x$ et $y$, telles que $a x^{2}+$ $2 b x y+c y^{2}$ soit premier $\dot{a} A$, et satisfaisant en outre $\dot{a}$ la condition (3). Quant aux autres termes, comme ils sont de même nature que celui dont nous venons de parler et résultent de ce dernier, en accentuant les lettres $a, b, c$, nous continuerons à ne pas les écrire. Il s'agit maintenant de transformer l'équation que nous venons d'obtenir, de manière à ce qu'elle exprime le nombre des formes non-équivalentes qui répondent au déterminant $D$. Ce sera là l'objet du $\$$ s. suivant.

Expression du nombre des classes au moyen d'une suite infinie double.

\$. 18.

Pour parvenir au but que nous avons en vue, nous aurons à examiner ce que les différents termes de l'équation (9) du $\$$. précédent de- 
viennent, lorsque la variable $s$ que cette équation contient, converge vers sa limite qui est l'unité.

I. Occupons-nous d'abord du second membre, en nous bornant toujours à considérer la première des sommes dont ce membre se compose. Comme indépendamment de la double condition d'inégalité à laquelle $\boldsymbol{x}$ et $\boldsymbol{y}$ sont supposés satisfaire, ces indéterminées doivent être telles que la valeur du trinome $a x^{2}+2 b x y+c y^{2}$ soit première à $\Delta$, on conclut du 5. 15, III, que les valeurs simultanées de $x$ et $y$, que la sommation embrasse, peuvent être distribuées en systèmes de la forme

$$
\text { (1) } x=\Delta v+\alpha, \quad y=\Delta w+\gamma \text {, }
$$

où $v, w ; \alpha, \gamma$ désignent des entiers complexes, les deux premiers indéterminés et les deux derniers déterminés, et que le nombre de ces systèmes est toujours $\boldsymbol{N}(\Delta) \psi(\Delta)$. La somme dont il s'agit se décompose ainsi ell $\boldsymbol{N}(\Delta) \psi(\Delta)$ sommes partielles, telles que la suivaite

$$
\text { (2) } \Sigma \frac{1}{\left(N\left(a x^{2}+2 b x y+c y^{2}\right)\right)^{s}} \text {, }
$$

où le signe sommatoire doit s'étendre à toutes les valeurs de $x$ et $y$, données par les formules (1), et en outre telles que l'on ait

(3) $\mathbf{N}(a x+(b-v D) y)<N\left(a x+(b+v D) y \leqq \sigma^{2} \mathbf{N}(a x+(b-v D) y)\right.$. Pour évaluer la somme partielle $(2)$, soit $z$ une variable positive, et proposons-nous de déterminer l'entier positif $\boldsymbol{Z}$ qui exprime combien de fois dans la somme dont il s'agit, l'expression $N\left(a x^{2}+2 b x y+c y^{2}\right)$ obtient une valeur non-supérieure à $z$. On sent que $Z$ est uue fonction discontinue très-compliquée de la variable $z$; mais il ne s'agira pas d'obtenir cette fonction avec une exactitude absolue, et il suffira de connaitre son

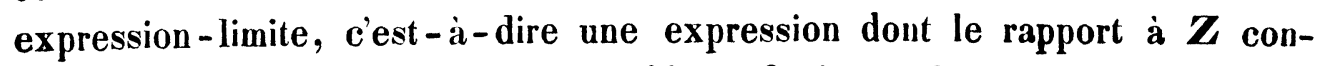
verge vers l'unité, lorsque la variable $z$ devient infinie. D'après ce qui précède, l'entier $\boldsymbol{Z}$ désigne le nombre des combinaisons $v, w$, pour lesquelles on a outre la condition (3), celle que nous allons écrire

$$
\boldsymbol{N}\left(\boldsymbol{a} \boldsymbol{x}^{2}+2 \boldsymbol{b} \boldsymbol{x} \boldsymbol{y}+\boldsymbol{c} \dot{y}^{2}\right) \leqq z,
$$

ou ce qui revient au même, celle-ci

$$
\text { (4) } \quad \boldsymbol{N}(a x+(b+\checkmark D) y) N(a x+(b-v D) y) \leqq N(a) s \text {, }
$$

$x$ et $y$ étant supposés remplacés dans les conditions $(3)$ et (4) par les expressions (1). 
Observons maintenant que, comme il ne s'agit que d'obtenir le nombre des combinaisons $v, w$, qui satisfont aux inégalités précédentes, nous pouvons remplacer les entiers $v, w$, par d'autres indéterminées $v^{\prime}, w^{\prime}$, entières ou uon, mais tellement liées à $v$ et $w$, qu'à toute combinaison $v, w$ réponde une combinaison unique $v^{\prime}, w^{\prime}$, et réciproquement. Soit pour abréger $z=$ $\frac{1}{\zeta^{4}}$, où $\zeta$ est supposé positif, il est facile de voir que nous remplirons la condition énoncée, en posant les équations linéaires $v^{\prime}=\zeta\left(v+\frac{\alpha}{\Delta}\right), w^{\prime}=$ $\zeta\left(w+\frac{\gamma}{\Delta}\right)$, en vertu desquelles, $\zeta$ étant réel et $v, w$ désignant des entiers complexes indéterminés, $v^{\prime}$ et $w^{\prime}$ exprimeront l'un et l'autre des nombres complexes, dont les deux parties sont les termes généraux de progressions arithmétiques réelles, ayant la quantité $\zeta$ pour raison commune. Au moyen de ces expressions les formules (1) que nous avons à substituer dans les conditions (3) et (4), deviennent $x=\frac{\Delta v^{\prime}}{\zeta}, y=\frac{\Delta w^{\prime}}{\zeta}$. Si maintenant l'on effectue la substitution dont il s'agit, et que l'on multiplie ensuite les inégalités (3) et (4) resp. par $\frac{\zeta^{2}}{N(\Delta)}$ et $\frac{\zeta^{4}}{N\left(\Delta^{2}\right)}$, il viendra simplement

$$
\left\{\begin{array}{l}
\boldsymbol{N}\left(a v^{\prime}+(b-V D) w^{\prime}\right)<\boldsymbol{N}\left(a v^{\prime}+(b+\checkmark D) w^{\prime}\right) \leqq \sigma^{2} \boldsymbol{N}\left(a v^{\prime}+(b-V D) v^{\prime}\right), \\
\boldsymbol{N}\left(a v^{\prime}+(b+V D) w^{\prime}\right) \boldsymbol{N}\left(a v^{\prime}+(b-V D) w^{\prime}\right) \leqq N\left(\frac{a}{d^{2}}\right)
\end{array}\right.
$$

de sorte que le nombre $\boldsymbol{Z}$ qu'il s'agit de déterminer, coïncide maintenant avec celui des combinaisons $v^{\prime}, w^{\prime}$, qui satisfont à ces dernières inégalités, dans lequelles $v^{\prime}$ et $w^{\prime}$ ont la signification indiquée plus haut. Il faut maintenant remplacer les nombres complexes, contenus dans ces inégalités, par leurs éléments réels. Posons pour cela

$$
\text { (6) } v^{\prime}=x+x^{\prime} i, \quad w^{\prime}=y+y^{\prime} i,
$$

où les quatre quantités réelles $x, x^{\prime}, y, y^{\prime}$, sont les termes généraux d'autant de progressions arithmétiques, indéfiniment prolongées dans les deux sens et dont la raison commune est $\zeta$. Posons encore

$$
\text { (7) } \quad a=\alpha+\alpha^{\prime} i, \quad b=\beta+\beta^{\prime} i, \quad \boldsymbol{V D}=\delta+\delta^{\prime} i,
$$
$\alpha, \alpha^{\prime}, \beta, \ldots$ étant des constantes réelles, et soit enfin pour abréger,

$$
\begin{cases}p=\alpha x-\alpha^{\prime} x^{\prime}, & q=\beta y-\beta^{\prime} y^{\prime}, \quad r=\delta y-\delta^{\prime} y^{\prime}, \\ p^{\prime}=\alpha^{\prime} x+\alpha x^{\prime}, & q^{\prime}=\beta^{\prime} y+\beta y^{\prime}, \quad r^{\prime}=\delta^{\prime} y+\delta y^{\prime} .\end{cases}
$$

En substituant les expressions (6) et (7), les inégalités (כ) prendront la forme 


$$
\left\{\begin{array}{c}
(p+q-r)^{2}+\left(p^{\prime}+q^{\prime}-r^{\prime}\right)^{2}<(p+q+r)^{2}+\left(p^{\prime}+q^{\prime}+r^{\prime}\right)^{2} \leqq \\
\sigma^{2}\left((p+q-r)^{2}+\left(p^{\prime}+q^{\prime}-r^{\prime}\right)^{2}\right), \\
\left((p+q+r)^{2}+\left(p^{\prime}+q^{\prime}+r^{\prime}\right)^{2}\right)\left((p+q-r)^{2}+\left(p^{\prime}+q^{\prime}-r^{\prime}\right)^{2}\right) \leqq N\left(\frac{a}{\Delta^{2}}\right) .
\end{array}\right.
$$

Il est maintenant facile de reconnaitre que l'entier $\boldsymbol{Z}$, lorsque la variable $\zeta$ dont il est fonction, devient infiniment petite, dépend de l'intégrale suivante

$$
\text { (10) } \iiint \int \partial x \partial x^{\prime} \partial y \partial y^{\prime}=A \text {, }
$$

dans laquelle les différentielles $\partial x, \partial x^{\prime}, \partial y, \partial y^{\prime}$ sont considérées comme positives, et qui est supposée s'étendre à toutes les valeurs des variables $x, x^{\prime}, y, y^{\prime}$, compatibles avec les conditions (9). En effet, si dans l'intégrale précédente l'on considère les quatre différentielles comıne constantes et égales à $\zeta$, tous les éléments de cette intégrale auront la valeur commune $\zeta^{*}$, de sorte que l'intégrale sera égale au produit de $\zeta^{4}$ par le nombre des combinaisons $x, x^{\prime}, y, y^{\prime}$, qui satisfont aux conditions (9), et dans lesquelles les variables sont supposées croître de la différence constante $\zeta$. Or, ce deruier nombre étant précisément l'entier $Z$, on aura pour une valeur infiniment petite de $\zeta, Z_{\zeta} \zeta^{4}=A$, et par suite $\mathbf{Z}=\frac{\boldsymbol{A}}{\zeta^{4}}$, ou ce qui revient au même,

$$
\text { (11) } \mathbf{Z}=\boldsymbol{A} z \text {, }
$$

z étant supposé infini. Il est encore facile de s'assurer qu'en même temps que le rapport des deux membres de cette dernière équation tend vers la limite 1, leur différence croît moins rapidement qu'une puissance de $z$, dont l'exposant constant serait tant soit peu supérieur $\dot{a}_{4}^{3}$, et généralement à $\frac{m-1}{m}$, s'il s'agissait d'une intégrale de l'ordre $m$ *) Tout se réduit donc maintenant $\grave{a}$ obtenir la valeur $\boldsymbol{A}$ de l'intégrale (10); pour y parvenir, on pourrait faire usage d'une substitution unique, mais le calcul

*) Le principe dont nous faisons usage dans le texte, est évident et résulte immédiatement de la notion mème d'une intégrale multiple, considerée comme une somme d'éléments infiniments petits, lorsque, comme il arrive ici, les variables ne doivent pas obtenir des valeurs infinies dans les intégrations qu'ıl s'agit d'effectuer; mais il est bon d'ajouter que, si, l'intégrale elle-mème restant toujours finie, cette dernière circonstance n'avait plus lieu, l'application du même principe pourrait conduire à des résultats entièrement erronnés, ce dont il est facile de voir la raison, et comme l'on peut d'ailleurs s'en assurer par des exemples, en considérant une intégrale double exprimant upe aire finie, comprise entre une courbe et son asymptote. Quant ̀̀ l'assertion que nous venons d'avancer et d'après laquelle les variables $x, x^{\prime}, y^{\prime}, y^{\prime}$ ne sauraient ètre infinies dans notre cas, elle résulte trop simplement des conditions (9), pour qu'il soit nécessaire de nous $y$ arz̧êter. 
devient beaucoup plus simple, si l'on emploie plusieurs substitutions successives. Observons que, comme l'ordre des intégrations est arbitraire, nous pouvons considérer les intégrations relatives $\dot{a} x$ et $x^{\prime}$, comme devant être effectuées les premières, et que rien ne s'oppose alors à ce que nous remplacions les variables $x$ et $x^{\prime}$ par de nouvelles variables $t$ et $t^{\prime}$, liées aux premières par des équations qui contiennent $y$ et $y^{\prime}$, pourvu que dans ces équations l'on traite $y$ et $y^{\prime}$ comme des constantes. Posons donc $t=$ $p+q, t^{\prime}=p^{\prime}+q^{\prime} ; p, q, p^{\prime}, q^{\prime}$ désignant les expressions linéaires (8). Si à ces deux équations l'on applique la formule connue qui sert à la transformation des intégrales doubles, on trouvera que le produit $\partial x \partial x^{\prime}$ devra être remplacé par $\frac{1}{\alpha^{2}+a^{\prime 2}} \partial t \partial t^{\prime}=\frac{1}{N(a)} \partial t \partial t^{\prime}$. En substituant cette dernière expression dans l'intégrale et les nouvelles variables dans les conditions (9), qui définissent l'étendue des intégrations, on aura

$$
\begin{gathered}
\iiint \int \partial t \partial t^{\prime} \partial y \partial y^{\prime}=\mathbf{N}(a) A, \\
(t-r)^{2}+\left(t^{\prime}-r^{\prime}\right)^{2}<(t+r)^{2}+\left(t^{\prime}+r^{\prime}\right)^{2}<\sigma^{2}\left((t-r)^{2}+\left(t^{\prime}-r^{\prime}\right)^{2}\right), \\
\left((t+r)^{2}+\left(t^{\prime}+r^{\prime}\right)^{2}\right)\left((t-r)^{2}+\left(t^{\prime}-r^{\prime}\right)^{2}\right)<N\left(\frac{a}{d^{2}}\right),
\end{gathered}
$$

où nous avons supprimé les signes d'égalité qui accompagnaient ceux d'inégalité et qui sont désormais inutiles, les conditions précédentes se rapportant maintenant à des variables continues. Si en second lieu, $t$ et $t^{\prime}$ étant considérés comme constants, nous remplaçons les variables $y$ et $y^{\prime}$, à leur tour par de nouvelles variables $r$ et $\boldsymbol{r}^{\prime}$, liées $\dot{a} y$ et $y^{\prime}$ par les deux dernières des formules $(8)$, l'intégrale deviendra

$$
\iiint \partial t \partial t^{\prime} \partial \boldsymbol{r} \dot{\partial} \boldsymbol{r}^{\prime}=N(a \vee D) A
$$

les conditions qui en définissent l'étendue, étant toujours celles que nous venons d'écrire. Distribuons actuellement les quatre variables en ces deux groupes $t, r ; t^{\prime}, r^{\prime}$, et remplaçons les resp. par ces deux nouveaux groupes $x, x^{\prime} ; y, y^{\prime}$, liés aux précédents par les équations

$$
x=t-r, \quad x^{\prime}=t+r ; \quad y=t^{\prime}-r^{\prime}, \quad y^{\prime}=t^{\prime}+r^{\prime}
$$

ell vertu desquelles il faudra mettre $\frac{1}{2} \partial x \partial x^{\prime}, \frac{1}{2} \partial y \partial y^{\prime}$ resp. a la place de $\partial t \partial \boldsymbol{r}, \partial t^{\prime} \partial \boldsymbol{r}^{\prime}$. Lintégrale et les conditions qui s'y rapportent, se cbangeront ainsi en

$$
\begin{gathered}
\iiint \int \partial x \partial y \partial x^{\prime} \partial y^{\prime}=4 \boldsymbol{N}(a \vee D) A \\
x^{2}+y^{2}<x^{\prime 2}+y^{\prime 2}<\sigma^{2}\left(x^{2}+y^{2}\right), \quad\left(x^{2}+y^{2}\right)\left(x^{\prime 2}+y^{\prime 2}\right)<N\left(\frac{a}{\Delta^{2}}\right) .
\end{gathered}
$$


Remplaçons mainteuant les variables de chacun des groupes $x, y ; x^{\prime}, y^{\prime}$ par des coordonnées polaires, en posant

$$
x=\rho \cos \theta, \quad y=\rho \sin \theta ; \quad x^{\prime}=\rho^{\prime} \cos \theta^{\prime}, \quad y^{\prime}=\rho^{\prime} \sin \theta^{\prime},
$$

où il importe de remarquer qu'indépendamment des conditions auxquelles les nouvelles variables doivent satisfaire eu vertu des inégalités précédentes, il faudra regarder $\rho$ comme positif et $\theta$ comme étant compris entre les limites 0 et $2 \pi$, pour qu'à une même combinaison $x, y$ ne réponde pas plus d'une combinaison $\rho, \theta$; et que $\rho^{\prime}, \theta^{\prime}$ doivent être assujettis à la même limitation. Par l'introduction de ces nouvelles variables, il viendra

$\iiint \rho \rho^{\prime} \partial \rho \partial \rho^{\prime} \partial \theta \partial \theta^{\prime}=4 \mathbf{N}(\boldsymbol{a} \vee \boldsymbol{D}) \boldsymbol{A}, \quad \rho^{2}<\varrho^{2}<\sigma^{2} \rho^{2}, \quad \rho^{2} \varrho^{\prime 2}<\boldsymbol{N}\left(\frac{a}{\Delta^{2}}\right)$.

Les conditions d'inégalité ne contenant pas les variables $\theta$ et $\theta^{\prime}$, les intégrations qui s'y rapportent, devront s'étendre depuis 0 jusqu'à $2 \pi$; en effectuant ces deux intégrations et remplaçant en outre $\rho^{2}, \rho^{\prime 2}$ resp. par $\rho, \rho^{\prime}$, de sorte que ces nouvelles variables devront être considerées comme positives, on trouvera

$$
\iint \partial \rho \partial \rho^{\prime}=\frac{4}{\pi^{2}} \boldsymbol{N}(a \boldsymbol{V D}) \boldsymbol{A}, \quad \rho<\rho^{\prime}<\sigma^{2} \rho, \quad \rho \rho^{\prime}<\boldsymbol{N}\left(\frac{a}{\Delta^{2}}\right) .
$$

Si maintenant, $\rho$ étant regardé comme constant, nous remplaçons $\rho^{\prime}$ par une nouvelle variable $\nu$, déterminée par l'équation $\rho^{\prime}=\nu \rho$, et qui en vertu de ce qui précède, doit être considérée comme positive, nous aurons d'abord

$$
\iint \rho \partial \rho \nu=\frac{4}{\pi^{2}} \boldsymbol{N}(\boldsymbol{a} \boldsymbol{V} \boldsymbol{D}) \boldsymbol{A}, \quad 1<\nu<\sigma^{2}, \quad \rho^{2}<\boldsymbol{N}\left(\frac{a}{J^{2}}\right) \frac{1}{\nu},
$$

et par suite, en effectuant l'intégration relative $\dot{a} \rho$, et qui doit s'etendre depuis $\varrho^{2}=0$ jusqu'à $\varrho^{2}=N\left(\frac{a}{d^{2}}\right) \frac{1}{v}$,

$$
\int \frac{\partial \nu}{\nu}=\frac{8}{\pi^{2}} \boldsymbol{N}\left(\Delta^{2} \vee \boldsymbol{D}\right) \boldsymbol{A}, \quad 1<\nu<\sigma^{2},
$$

d'où l'on conclut enfin

$$
\boldsymbol{A}=\frac{\pi^{2} \log \sigma}{4 N\left(\Delta^{2} \sqrt{D}\right)}
$$

Après avoir ainsi déterminé le coëfficient $\boldsymbol{A}$, contenu dans l'équation (11), il sera facile de voir ce que la somme partielle (2) devient, lorsque l'exposant $s$ converge vers l'unité, ou ce qui revient au même, lorsque la variable positive $\rho$, supposée liée à $s$ par l'équation $s=1+\rho$, est considérée comme infiniment petite. En effet, comme la fonction $\boldsymbol{Z}$, qui exprime combien de fois dans la somme en question, l'expression $N\left(a x^{2}+2 b x y+c y^{2}\right)$ 
obtient une valeur qui ne surpasse pas celle de $\approx$, est telle que les deux rapports $\frac{Z}{A z}, \frac{Z-A z}{z^{\gamma}}$, où $\gamma$ désigne une constante supérieure à la fraction $\frac{3}{4}$, convergent le premier vers une limite égale à l'unité, le second vers la limite zéro, lorsque la variable $z$ devient plus grande que toute grandeur donnée, on conclut sur le champ du lemme démontré dans le $\mathfrak{\$} .1$. du Mémoire déjà plusieurs fois cité, que pour une valeur infiniment petite de $\rho$, la somme (2) prend cette forme très-simple

$$
\left.\frac{A}{\rho}=\frac{\pi^{2} \log \sigma}{4 N\left(\Delta^{2} \frac{1}{\sqrt{D})}\right.} \cdot \frac{1}{\varrho} *\right) \text {. }
$$

Comme cette expression ne présente rien que soit particulier à la somme partielle que nous avous considérée, ni même rien qui soit particulier à la somme totale dont cette somme partielle fait partie, puisqu'elle n'est - fonction que du seul déterminant $\boldsymbol{D}$, commun à toutes les formes quadratiques contenues dans le second membre de l'équation (9) $\mathbf{\$} . \mathbf{1 7}$, l'on voit qu'il suffit de la multiplier par le nombre $\boldsymbol{N}(\Delta) \psi(\Delta)$, qui est celui des sommes partielles contenues dans une même somme totale, et par celui des formes qui constituent un système complet relativement au déterminant $D$, pour en conclure la valeur du second membre de l'équation dont il s'agit, pour le cas où l'on y considère $\varrho$ comme infiniment petit. Il viendra ainsi

$$
\text { (12) } \frac{\pi^{2} \psi(\Delta) \log \sigma}{4 N(\Delta \sqrt{D})} \boldsymbol{H} \frac{1}{\varrho}
$$

$\boldsymbol{H}$ désignant le nombre des classes qui répondent au déterminant $\boldsymbol{D}$.

II. Il s'agit maintenant de considérer le premier membre de l'équation citée. Ce membre pouvant se mettre sous la forme

$$
4 \Sigma \frac{1}{(N n)^{s}} \times 2 \Sigma\left[\frac{D}{n}\right] \frac{1}{(N n)^{s}},
$$

*) Quoique les deux propriétés dont nous venons de faire usage, ressortent l'une et l'autre avec évidence des considérations indiquées plus haut, il peut être bon de faire remarquer que la première de ces propriétés suffit à elle seule pour en tirer la conclusion que nous venons d'énoncer. C'est ce qui résulte d'une remarque déjà faite dans le Mémoire précédent, et d'après laquelle le lemme dont il s'agit, comporte plus d'étendue qu'il n'a été nécessaire de lui donner à l'endroit cité. Il est en effet facile de reconnaitre que la vérité de ce lemme ne suppose qu'une seule condition, consistant en ce que la fonction, désignée par $f(t)$ dans son énoncé, doit ètre telle que l'on ait $\frac{f(t)}{t}=c$, lorsque $t$ obtient une valeur infinie. Pour s'en assurer, on n'aura qu'à apporter une modification assez légère et qui se présente facilement, à la démonstration qui a été exposée daus le précédent Mémoire. 
occupons-nous d'abord du premier de ces deux facteurs. Comme la somme dont il s'agit, doit s'étendre à tous les entiers $n$, premiers à $\Delta$ et en outre primaires, ils est évident que nous pouvons faire abstraction de la dernière de ces deux conditions, pourvu qu'en même temps nous omettions le facteur 4. Les valeurs que $\boldsymbol{n}$ doit recevoir, peuvent se distribuer en systèmes de la forme $n=\Delta v+\alpha, v$ et $a$ désignant des entiers complexes, le premier indéterminé, le second déterminé pour chaque système, et devant être égalé successivement à tous ceux des termes d'un système de résidus pour le module $\Delta$, qui n'ont pas de diviseur commun avec ce module. Considérons la somme partielle, répondant à l'un quelconque de ces termes, et qui est

$$
\Sigma \frac{1}{(N(\Delta v+\alpha))^{1+\rho}} \text {. }
$$

Pour en obtenir la valeur, soit $\boldsymbol{z}$ une variable positive et $\boldsymbol{Z}$ la fonction discontinue de cette variable, qui exprime le nombre des entiers $v$ pour lesquels on a $N(\Delta v+\alpha) \leqq z$. Si nous posons, pour abréger, $z=\frac{1}{\zeta^{2}}, \zeta$ étant positif, et que nous remplacions $v$ par une nouvelle indéterminée $v^{\prime}$, telle qu'on ait $v^{\prime}=\zeta\left(v+\frac{\alpha}{\Delta}\right)$, l'inégalité précédente se changera en $N\left(v^{\prime}\right) \leqq \frac{1}{N(\Delta)}$, et $\boldsymbol{Z}$ désignera alors le nombre des valeurs $\boldsymbol{v}^{\prime}$ qui satisfont $\dot{a}$ cette dernière. Or, comme en posant $v^{\prime}=x+x^{\prime} i, x$ et $x^{\prime}$ seront évidemment les termes généraux de deux suites dont la première différence est constante et égale $\dot{\mathbf{a}} \zeta$, on voit, comme plus haut, que pour une valeur infinie de $z$, l'on aura $Z=B z, B$ désignant l'intégrale $\iint \partial x \partial x^{\prime}, x^{2}+x^{\prime 2}<\frac{1}{N(\Delta)}$. Mais cette deruière étant évidemment égale à $\frac{\pi}{N(\Delta)}$, on conclura du lemme déjà employé dans le précédent $n^{\circ}$, que la somme partielle que nous considérons, se réduit simplement à $\frac{\pi}{N(\Delta)} \frac{1}{\rho}$, lorsque la variable $\rho$ est supposée devenir moindre que toute grandeur donnée; d'où il suit enfin

$$
\text { (13) } 4 \Sigma \frac{1}{(N n)^{1+\varrho}}=\frac{\pi \psi(\Delta)}{N(\Delta)} \cdot \frac{1}{\varrho} \text {, }
$$

le signe s'étendant, comme dans l'équation (9) du \$. 18, à tous les entiers $n$, premiers ḋ $\Delta$ et en outre primaires.

III. Pour mettre enfin la seconde des deux sommes rappelées au commencement $d u n^{\circ}$ précédent, sous la forme appropriée à notre but, il faut distinguer plusieurs cas différents que le déterminant $\boldsymbol{D}$ peut présenter. 
Observons pour cela que, si nous réunissons en un seul quarré tous les facteurs doubles de $D$, nous pourrons toujours mettre cet entier sous la forme (14) $D=\chi Q V^{2}$,

$\chi$ ayant l'une de ces quatre valeurs

(15) $x=1, \quad x=i, \quad x=1+i, \quad x=i(1+i)$,

et $\boldsymbol{Q}$ ou $-\boldsymbol{Q}$ désignant un produit de facteurs simples impairs et primaires, tous inégaux, sans exclure le cas où l'on aurait $Q= \pm 1$, qui ne peut toutefois avoir lieu qu'autant qu'on n'a pas $\chi=1$, les déterminants quarrés étant toujours exclus. Nous ajouterons que les entiers $\chi, \boldsymbol{Q}, \boldsymbol{V}$, s'ils doivent être tels que nous venons de les définir, seront complètement déterminés pour tout déterminant donné, si ce n'est que $\boldsymbol{Q}$ et $V^{2}$ peuvent être simultanément remplacés par $-\boldsymbol{Q}$ et $(\boldsymbol{V} i)^{2}$. Cela posé, nous allons transformer l'expression $\left[\frac{D}{n}\right]$ au moyen des équations $(e)$ et $(f)$ du $\$$. 8. On a d'abord évidemment en vertu des équations citées,

$$
\left[\frac{D}{n}\right]=\left[\frac{\chi Q V^{2}}{n}\right]=\left[\frac{\chi}{n}\right]\left[\frac{Q}{n}\right]=\left[\frac{\chi}{n}\right]\left[\frac{n}{Q}\right] .
$$

Pour transformer le facteur $\left[\frac{\chi}{n}\right]$, il devient nécessaire d'introduire explicitement les deux entiers réels contenus dans $n$; posons donc $n=\lambda+v i$, $\lambda$ et $\nu$ étant resp. des formes $4 k+1,2 k$. Il viendra alors suivant les quatre cas déjà distingués (15), et en ayant égard aux deux premières des équations $(f)$ citées,

$$
\begin{aligned}
& {\left[\frac{\chi}{n}\right]=1, \quad\left[\frac{\chi}{n}\right]=(-1)^{\frac{\lambda^{2}+\nu^{2}-1}{4}}, \quad\left[\frac{\chi}{n}\right]=(-1)^{\frac{(\lambda+v)^{2}-1}{8}},}
\end{aligned}
$$

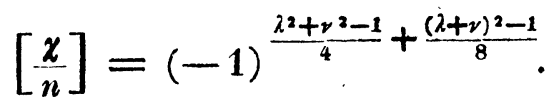

Pour réunir ces quatre expressions en une seule formule, nous poserons $\delta= \pm 1, \varepsilon= \pm 1$, les signes ambigus étant choisis suiyant les quatre cas que $\chi$ peut présenter en vertu des équations (15), comme il suit

(16) $\delta=1, \varepsilon=1 ; \quad \delta=-1, \varepsilon=1 ; \quad \delta=1, \varepsilon=-1$;

$$
\delta=-1, \varepsilon=-1 \text {. }
$$

Cette convention admise, nous aurons pour tous les cas

$$
\left[\frac{\chi}{n}\right]=\delta^{\frac{\lambda x^{2}+\nu^{2}-1}{4}} \varepsilon^{\frac{(\lambda+\nu)^{2}-1}{8}} .
$$

Au moyen de cette dernière expression et de celles déjà obtenues; le facteur du premier membre de l'équation (9) (\$. 7.), qu'il s'agissait de trans- 
former, prendra la forme

$$
2 \Sigma\left[\frac{D}{n}\right] \frac{1}{(\boldsymbol{N} n)^{1+\varphi}}=2 \Sigma \delta^{\frac{\lambda^{2}+\nu^{2}-1}{4}} \frac{(\lambda+\nu)^{2}-1}{\varepsilon}\left[\frac{\lambda+\nu i}{Q}\right] \frac{1}{\left(\lambda^{2}+\nu^{2}\right)^{1+\varphi}} .
$$

IV. Si maintenant nous substituons les expressions (12), (13) et (17) dans l'équation citée, et que nous effaçions le facteur $\frac{1}{\varrho}$ et les autres facteurs, communs aux deux membres, l'équation dont il s'agit, prendra la forme :

$$
\boldsymbol{H}=\frac{8 \boldsymbol{N}(\boldsymbol{V} \boldsymbol{D})}{\pi \log \sigma} \Sigma \delta^{\frac{\lambda^{2}+\nu^{2}-1}{4}} \varepsilon^{\frac{(\lambda+\nu)^{2}-1}{8}}\left[\frac{\lambda+\nu i}{Q}\right] \frac{1}{\left(\lambda^{2}+\nu^{2}\right)^{1+Q}}
$$

Telle est la suite infinie double qui exprime le nombre des classes pour un déterminant quelconque non-quarré $D$, et dans laquelle nous avons conservé la quantité infiniment petite $\varrho$, qui ne doit être annulée qu'après que l'on aura fixé l'ordre dans lequel les termes de la double somme doivent se suivre, pour que cette somme soit en effet la limite de celle qui répond à une valeur infiniment petite de la variable positive $\rho$. La signification des lettres qui entrent dans l'équation, a été fixée dans ce qui précède, et l'on devra se rappeler que la double sommation doit s'étendre à tous les couples d'entiers réels $\lambda$ et $\nu$, respectivement des formes $4 k+1$, $2 \boldsymbol{k}$, et tels que l'entier complexe correspondant $\lambda+\nu \boldsymbol{i}$ soit premier à $\boldsymbol{D}$.

Pour effectuer la double sommation, il faudra d'abord transformer le facteur $\left[\frac{\lambda+\nu i}{Q}\right]$ au moyen de l'équation $(h)$ du $\$ .8$, et remplacer ensuite les nouveaux symboles ainsi introduits, par une suite finie de sinus ou de cosinus, en se servant pour cet objet des formules connues, dues a Mr. Gaufs. Après ces deux substitutions, l'une dè deux sommations pourra être exécutée au moyen d'une suite trigonométrique, dont la somme a été donnée par Euler, et la suite infinie double, réduite par-là à une série simple, se décomposera alors en plusieurs séries partielles qui rentrent dans celles par lesquelles Abel et Mr. Jacobi ont développé les fonctions trigonométriques de l'amplitude d'une fonction elliptique de première espèce. Mais si avec le secours des formules dont les illustres géomètres que nous venons de citer, ont enrichi l'Analyse, la sommation en elle-même ne présente pas de difficulté réelle et n'exige que peu d'espace, il n'en est pas de même de la discussion à laquelle il faut soumettre le résultat qui s'en déduit, pour en reconnaître la véritable nature. Comme le résultat dont il s'agit, se trouve dépendre de la division en parties égales de la fonction $48 *$ 
elliptique complète de première espèce, pour le cas où le module a la valeur $\sqrt{\frac{1}{2}}$, et où le nombre de ces parties égales est un entier complexe; et que la théorie des équations algébriques, qui se rapportent à une telle division, n'a été qu'ébauchèe jusqu'à présent * $)$ : il sera nécessaire d'entrer à cet égard dans de nouveaux développements dont l'étendue excéderait de beaucoup les bornes que nous avons dû imposer à cette première partie de notre travail. C'est pourquoi et comme nous en avons déjà averti, nous réserverons ces détails pour la seconde partie, et nous terminerons celle-ci par l'examen des deux cas particuliers, déjà mentionnés dans le préambule du présent Mémoire.

\section{Examen de deux cas particuliers.}

\section{19.}

Les deux cas qu'il s'agit de considérer, sont ceux où le déterminant $D$ est un entier réel ou le produit d'un tel entier par $i$. Comme en vertu de l'équation (18) du $\$$. précédent, le nombre des classes est évidemment le même pour deux déterminants opposés, nous pourrons toujours considérer comme positif, l'entier dont il vient d'être question.

I. Soit en premier lieu $\boldsymbol{D}$ un entier positif non-quarré, et soit $\boldsymbol{S}^{2}$ le plus grand quarré réel qui divise $D$. Nous aurons alors l'un de ces deux cas

$$
\text { (1) } \quad \boldsymbol{D}=\boldsymbol{P} \boldsymbol{S}^{2}, \quad \boldsymbol{D}=2 \boldsymbol{P} \boldsymbol{S}^{2}
$$

$\boldsymbol{P}$ désignant un produit de nombres premiers positifs, impairs et tous inégaux, produit qui peut d'ailleurs se réduire à l'unité dans le second des deux cas précédents. Comme, en considérant $\boldsymbol{P}$ comme complexe, cet entier ou son opposé est primaire et n'a que des facteurs simples inégaux, il suffit de mettre la seconde des équations (1) sous la forme $D=i P((1-i) S)^{2}$, pour reconnaître que les équations $(14),(15)$ et (16) du $\$ .18$, qui se rapportent à un déterminant quelconque, donnent relativement au cas particulier qui nous occupe, $\boldsymbol{Q}=\boldsymbol{P}, \varepsilon=1, \delta= \pm 1$, où il faut choisir le signe supérieur ou le signe inférieur dans la dernière de ces équations, selon que $D$ présente le premier ou le second des deux cas (1). En substituant ces valeurs dans l'expression générale de $\boldsymbol{H}$, et remplaçant en même temps $\sigma$ par sa valeur,

*) Voir un Mémoire d'Abel, inséré dans ce Jourual, Tome III, pag. 160. 
donnée par la formule (3) du \$. 14, ainsi que $\left[\frac{\lambda+\nu i}{P}\right]$ par l'expression équivalente, fournie par la première des équations $(g)$ du $\$ .8$, il viendra

$$
\boldsymbol{H}=\frac{8 D}{\pi x \log (\tau+v \sqrt{D})} \Sigma \delta^{\frac{\lambda^{2}+\nu^{2}-1}{4}}\left(\frac{\lambda^{2}+\nu^{2}}{\boldsymbol{P}}\right) \frac{1}{\left(\lambda^{2}+\nu^{2}\right)^{1+\rho}},
$$

de sorte que la double somme ne contient plus l'entier complexe $\lambda+v i$, mais seulement sa norme $\lambda^{2}+v^{2}$. II est vrai que cet entier semble $\mathbf{y}$ entrer encore implicitement par la condition, en vertu de laquelle $\lambda+\nu i$ doit être premier $\dot{a} \boldsymbol{D}$; mais ce dernier entier étant réel, on voit que la condition dont il s'agit, revient à celle que $\boldsymbol{D}$ et $\lambda^{2}+\nu^{2}$ doivent être sans diviseur commun.

II. Considérons en second lieu un déterminant de la forme, $D=D^{\prime} i$, $D^{\prime}$ étant un entier positif qu'il faudra seulement supposer tel que $2 D^{\prime}$ ne soit pas un quarré, sans quoi $D$ serait lui-même un quarré. Si nous désignons par $\boldsymbol{S}^{\prime 2}$ le plus grand quarré réel qui divise $2 \boldsymbol{D}^{\prime}$, nous aurons l'une ou l'autre de ces deux équations,

$$
\text { (3) } 2 \boldsymbol{D}^{\prime}=\boldsymbol{P}^{\prime} \boldsymbol{S}^{\prime 2}, \quad 2 \boldsymbol{D}^{\prime}=2 \boldsymbol{P}^{\prime} \boldsymbol{S}^{\prime 2} \text {, }
$$

dans lesquelles $\boldsymbol{P}^{\prime}$ est un produit de nombres premiers positifs, impairs et inégaux, et $S^{\prime}$ étant pair dans la première de ces deux équations. Ces équations donnent respectivement celles-ci,

$$
\boldsymbol{D}=\boldsymbol{P}^{\prime}\left((1+i)_{\frac{1}{2}} \boldsymbol{S}^{\prime}\right)^{2}, \quad \boldsymbol{D}=\boldsymbol{P}^{\prime} \boldsymbol{i} \boldsymbol{S}^{\prime 2},
$$

qu'il suffit de comparer aux équations déjà citées du \$.18, pour voir que nous avons $\boldsymbol{Q}=\boldsymbol{P}^{\prime}, \varepsilon=1, \delta= \pm 1$, le signe supérieur ou le signe inférieur devant être choisi, selon que $2 \boldsymbol{D}^{\prime}$ présente le premier ou le second des deux cas (3). Au moyen de ces valeurs et par des transformations analogues à celles que nous avous opérées dans le $n^{\circ}$ précédent, l'on trouvera

$$
\text { (4) } \boldsymbol{H}=\frac{8 D^{\prime}}{\pi x \log \left(\tau+v \sqrt{ } 2 D^{\prime}\right)} \sum \delta^{\frac{\lambda^{2}+\nu^{2}-1}{4}}\left(\frac{\lambda^{2}+\nu^{2}}{\boldsymbol{P}^{\prime}}\right) \frac{1}{\left(\lambda^{2}+\nu^{2}\right)^{1+\rho}} \text {, }
$$

$u, \tau, v$ ayant la même signification que dans l'équation (4) du $\$ .14$, et la double sommation devant s'étendre à tous les couples d'entiers réels $\lambda, \nu$, resp. compris dans les formes $4 k+1,2 k$, et tels que $\lambda^{2}+\nu^{2}$ soit premier à $\boldsymbol{D}^{\prime}$.

III. Nous allons maintenant faire voir que les sommes doubles (2) et (4) peuvent être remplacées chacune par un produit de. deux séries 
simples. La transformation qu'il s'agit d'effectuer, n'est qu'une application très-particulière de certaines équations générales dont nous avons eu à faire usage dans le précédent Mémoire $(\$ .6, V$.$) ; mais, pour mieux faire$ sentir le principe sur lequèl cette transformation repose, il nous paraît préférable de la rattacher à un théorème arithmétique très-simple et susceptible d'une démonstration tout élémentaire. Voici en quoi consiste ce théorème: "m désignant un entier positif et impair donné, le nombre des so"lutions de l'équation $m=x^{2}+y^{2}$, dans laquelle $x$ et $y$ sont des entiers „,réels indéterminés, est égal au quadruple excès du nombre des diviseurs „(positifs) de $m$, qui sont compris dans la forme $4 k+1$, sur celui de ces „, diviseurs qui ont la forme $4 k+3$. ." *)

Comme les entiers $x$ et $y$, qui satisfont, à l'équation précédente, sont toujours l'un pair, l'autre impair, on voit que, si l'on considère l'un de ces entiers, le second par exemple, comme devant être pair, le nombre des solutions se réduira de moitié, et l'on voit également que, si l'on suppose en outre $x$, pris avec son signe, de la forme $4 k+1$, le nombre des solutions éprouvera une seconde réduction de même étendue, et deviendra simplement égal à l'excès défini dans l'énoncé, puisqu'à une même valeur de $y$, répondent toujours deux valeurs opposées de $x$, qui sont l'une de la forme $4 k+1$, l'autre de celle-ci $4 k+3$. Au moyen de ce résultat, il est facile de former l'équation générale que nous allons écrire,

$$
\Sigma \boldsymbol{F}\left(\boldsymbol{x}^{2}+y^{2}\right)=\Sigma(-1)^{\frac{n-1}{2}} \boldsymbol{F}\left(n n^{\prime}\right),
$$

et dans laquelle la double sommation, indiquée dans le premier membre, doit s'étendre à tous les entiers positifs ou négatifs $x$ et $y$, resp. compris dans les formes $4 k+1,2 k$, tandis que celle du second membre est supposée embrasser tous les entiers impairs et positifs, $n$ et $\boldsymbol{n}^{\prime}$. D'après la manière dont cette équation subsiste, il est encore évident qu'elle ne cessera pas d'avoir lieu, si aux conditions énoncées nous ajoutons celles que $x^{2}+y^{2}$ soit premier à un entier réel donné $\boldsymbol{K}$, et qu'il en soit de même du produit $\boldsymbol{n} n^{\prime}$, ces uouvelles conditions n'ayant d'autre effet que de supprimer les mêmes termes de part et d'autre. Ainsi restreintes, les indéterminées $x$ et $y$ auront la même signification que celles désiguées par $\lambda$ et $\nu$ dans les sommes (2) et (4), en supposant respectivement $K=D$, ou $K=D^{\prime}$,

*) Voyez pour la démonstration de ce théorème, dû à Mr. Jacobí, le Tome XII de ce Jaurnal, pag. 16\%, ou le Mémoire cité, $\$ 7$. 
tandis que $n$ et $n^{\prime}$ devront être resp. supposés premiers à $D$ ou à $D^{\prime}$. Cela posé, si dans les deux sommes (2) et (4) nous remplaçons l'exposant de $\delta$, par le produit $\frac{\lambda^{2}+\nu^{2}-1}{4} \cdot \frac{\lambda^{2}+\nu^{2}+1}{2}=\frac{\left(\lambda^{2}+\nu^{2}\right)^{2}-1}{8}$, ce qui est permis, le facteur ajouté étant impair, il suffira de supposer la fonction arbitraire $\boldsymbol{F}(\boldsymbol{z})$ de la forme,

$$
\delta^{\frac{z^{2}-1}{8}}\left(\frac{z}{P}\right) \frac{1}{z^{1+\rho}}
$$

pour conclure de l'équation en question, que la somme double (2), est équivalente à celle-ci

$$
\Sigma(-1)^{\frac{n-1}{2}} \delta^{\frac{\left(n n^{\prime}\right)^{2}-1}{s}}\left(\frac{n n^{\prime}}{\mathrm{P}}\right) \frac{1}{\left(n n^{\prime}\right)^{1+\rho}},
$$

où le signe $\Sigma$ se rapporte à tous les entiers positifs $n$ et $n^{\prime}$, impairs et premiers à $D$. Observons maintenant que, l'exposant de $\delta$ étant toujours pair ou impair en même temps que le nombre $\frac{n^{2}-1}{8}+\frac{n^{2 \prime}-1}{8}$, comme nous avons déjà eu occasion de le remarquer dans le $\$ .8$, nous pouvons le remplacer par ce dernier. Par cette substitution, le terme général de la somme précédente se changera en un produit de deux facteurs qui ne contiennent chacun qu'un seul des entiers $n$ et $n^{\prime}$, de sorte que la somme ellemême prendra la forme d'un produit de deux séries simples. On obtient ainsi et en substituant dans l'équation (2), celle que nous allons écrire:

(2) $\quad \boldsymbol{H}=\frac{8 D}{\pi x \log (\tau+v \sqrt{D})} \Sigma \delta^{\delta^{2}-1}\left(\frac{n}{P}\right) \frac{1}{n} \cdot \Sigma(-1)^{\frac{n-1}{2}} \delta^{n^{2}-1}\left(\frac{n}{P}\right) \frac{1}{n}$, chacune des deux sommations indiquées s'étendant à tous les entiers positifs $n$, inpairs et premiers à $D$. Pour plus de simplicité, nous avons remplacé $n^{\prime}$ par $n$, ce qui est permis, ces deux lettres ayant la même signification et ne se trouvant plus maintenant mèlées dans une même sommation, et nous avons en outre réduit $\dot{a}$ zéro la variable infiniment petite $\rho$; les sommes précédentes étant en effet les limites de celles où l'on aurait conservé la quantité $\rho$, pourvu que dans ces sommes l'on considère les entiers $n$ comme formant une suite croissante, comme il est facile de s'en assurer, et comme on l'a d'ailleurs prouvé, en établissant les résultats qu'il sera nécessaire de rappeler dans le $\mathrm{n}^{\circ}$ suivant. L'équation (4) étant soumise aux mèmes transformations, se changera en celle-ci :

$$
\boldsymbol{H}=\frac{8 \boldsymbol{D}^{\prime}}{\pi \times \log \left(\tau+v \sqrt{2} \boldsymbol{D}^{\prime}\right)} \sum \delta^{\frac{n^{2}-1}{8}}\left(\frac{n}{\bar{P}}\right) \frac{1}{n} \cdot \Sigma(-1)^{\frac{n-1}{2}} \delta^{\frac{n^{2}-1}{8}}\left(\frac{n}{\bar{P}^{\prime}}\right) \frac{1}{\bar{P}^{\prime}} .
$$


IV. Il faut maintenant rappeler les résultats qui se rapportent aux formes quadratiques à coëfficients réels, pour les comparer à ceux que nous venons d'établir. C'est ce que nous allons faire, en choisissant les notations de manière à faciliter la comparaison dont il s'agit. Dáns le Mémoire précédent (\$.6, équat. 23), on a démontré que relativement à un déterminant positif non-quarré $D$, le nombre des classes que nous désignerons par $h_{1}$, est donné par l'équation

$$
\text { (5) } \quad h_{1}=\frac{2 \sqrt{D}}{\log (\tau+v \sqrt{D})} \Sigma \theta^{\frac{n-1}{2}} \delta^{\frac{n^{2}-1}{8}}\left(\frac{n}{P}\right) \frac{1}{n} \text {. }
$$

Dans cette équation la signification des lettres $\tau, v, \delta, \boldsymbol{P}$ et $n$, est la même que dans les équations précédentes (1), (2) et $\left(2^{\prime}\right)$, et la sommation a la même étendue que celles indiquées dans la dernière de ces équations. Quant à la lettre $\theta$, elle désigne l'unité positive ou négative, selon que $\boldsymbol{P}$ est de la forme $4 k+1$ ou de celle-ci $4 k+3$. Si nous considérons en second lieu le déterminant opposé $-D$, et que nous dénotions par $h_{2}$ le nombre des classes qui y répondeut, il résulte de l'équation (19) du $\$$. déjà cité, qu'ou aura

$$
\text { (6) } \quad h_{2}=\frac{2 \sqrt{D}}{\pi} \Sigma(-\theta)^{\frac{n-1}{2}} \delta^{\frac{n^{2}-1}{8}}\left(\frac{n}{P}\right) \frac{1}{n},
$$

la signification de toutes les lettres et l'étendue de la sommation restant toujours les mêmes. Or, $\theta$ élant toujours de la forme \pm 1 , l'on voit que les deux séries contenues dans les équations ( 5 ) et ( 6 ), coïncident avec celles de l'équation $\left(2^{\prime}\right)$, de sorte qu'en divisant cette dernière par le produit des deux autres (5) et (6); on trouvera ce résultat très-simple

$$
\boldsymbol{H}=\frac{2}{x} h_{1} h_{2} \text {. }
$$

L'autre cas qui est celui d'un déterminani de la forme $D^{\prime} i$, conduit $\dot{a}$ un résultat analogue; il suffit pour l'obtenir, de remplacer dans ce qui précède, les équations (5) et (6), par celles au moyen desquelles s'expriment les nombres $h_{1}$ et $h_{2}$ des classes réelles qui répondent aux déterminants $2 D^{\prime}$ et $-2 D^{\prime}$. On trouve alors

$$
H=\frac{1}{x} h_{1} h_{2} \text {. }
$$

Nous avous donc ces deux théorènes très-remarquables.

„D désignant un éntier positif non-quarré, soit $\boldsymbol{H}$ le nombre des classes dans lesquelles se distribuent les formes à coëfficients complexes 
et au déterininant $D$, soient encore $h_{1}$ et $h_{2}$ les nombres des classes pour les formes à coëfficients réels, répondant respectivement aux deux déterminants $\boldsymbol{D}$ et $-\boldsymbol{D}$; toutes ces formes étant supposées telles que les coëfficients extrêmes et le double coëfficient moyen ne présentent pas de diviseur commun. Cela étant, l'on aura toujours $H=2 h_{1} h_{2}$, ou $H=h_{1} h_{2}$, selon que l'équation indéterminée $t^{2}-D u^{2}=-1$, admettra des solutions réelles ou non."

„D désignant un entier positif dont le double ne soit pas un quarré, si l'on suppose que les lettres $H, h_{1}, h_{2}$, en conservant une signification analogue à celle de l'énoncé précédent, se rapportent maintenant aux déterminants $D i, 2 D,-2 D$, on aura $H=h_{1} h_{2}$, ou $2 \boldsymbol{H}=h_{1} h_{2}$, selon que l'équation $t^{2}-2 D u^{2}=-1$, admettra des solutions réelles ou non." *)

Quoique les théorèmes précédents ne contiennent aucun élément qui ne soit relatif aux nombres entiers, il parait difficile de les établir par des considérations purement arithmétiques, tandis que la méthode mixte dont nous venons de faire usage, et qui est fondée en partie sur l'emploi de quantités variant par degrés insensibles, nous y a conduit de la manière la plus naturelle et, pour ainsi dire, sans effort.

*) On suppose tacitement dans ces énoncés, comme on le fait ordinairement, que pour un déterminant négatif on n'admette que des formes réelles dont les coëfficients extrêmes soient positifs. Si on n'adoptait pas cet usage, le nombre $h_{2}$ aurait une valeur double de celle que nous lui supposons, et les deux énoncés seraient à modifier en conséquence.

Fautes à corriger dans ce Mémoire.

$$
\begin{aligned}
& \text { Page } 284 \text { ligne } 20 \text { étendues lisez étendus } \\
& \text { - } 296 \text { - } 20 \text { donc } l \text {. dont } \\
& \text { - } 298-25 \text { douc } i \text {. donc } \\
& \text { - } 301-29 \text { appelerons } l \text {. appellerons } \\
& \text { - } 306 \text { - } 8 \text { ou } l \text {. au } \\
& \text { _ } \quad \text { - 18, } 25 \text { à } \mu \text { l. à } m \\
& \text { - } 336-29 \text { connues } 1 \text {. continues } \\
& \text { - } 337 \text { - } 5 \text { ces } \text { l. ses } \\
& \text { - } \quad 7 \text { Car } l \text {. ; car } \\
& \text { - } 348 \text { - } 18 \text { ce nous } l \text {. ce que nous } \\
& -354-17\left[\frac{1}{(N q)^{s}}\right] \text { l. } \frac{1}{(N q)^{s}} \\
& \text { - } 359 \text { - } 27 \text { infiniments } l \text {. infiniment } \\
& \text { - } 362-9 \text { que } \text { l. qui } \\
& -369-31 \frac{1}{P^{\prime}} l \cdot \frac{1}{n}
\end{aligned}
$$

Crelle's Journal f. d. M. Bd. XXIY. Heft 4. 